\title{
The association of smoking status with SARS-CoV-2 infection, hospitalisation and mortality from COVID-19: A living rapid evidence review with Bayesian meta-analyses (version 8)
}

\author{
David Simons ${ }^{1}$, Lion Shahab², Jamie Brown ${ }^{2}$, Olga Perski $^{2}$ \\ 1 Royal Veterinary College, RVC \\ 2 University College London, University of London
}

Funding: The author(s) received no specific funding for this work.

Potential competing interests: The author(s) declared that no potential competing interests exist.

\section{Abstract}

Aims: To estimate the association of smoking status with rates of i) infection, ii) hospitalisation, iii) disease severity, and iv) mortality from SARS-CoV-2/COVID-19 disease.

Design: Living rapid review of observational and experimental studies with random-effects hierarchical Bayesian metaanalyses. Published articles and pre-prints were identified via MEDLINE and medRxiv.

Setting: Community or hospital. No restrictions on location.

Participants: Adults who received a SARS-CoV-2 test or a COVID-19 diagnosis.

Measurements: Outcomes were SARS-CoV-2 infection, hospitalisation, disease severity and mortality stratified by smoking status. Study quality was assessed (i.e. 'good', 'fair' and 'poor').

Findings: Version 8 (searches up to 22 September 2020) included 256 studies with 36 'good' and 'fair' quality studies included in meta-analyses. Sixty-seven studies (26.2\%) reported current, former and never smoking status with the remainder using broader categories. Recorded smoking prevalence among people with COVID-19 was generally lower than national prevalence. Current compared with never smokers were at reduced risk of SARS-CoV-2 infection $(R R=0.72,95 \%$ Credible Interval $(\mathrm{Crl})=0.57-0.89, \tau=0.40)$. Data for former smokers were inconclusive $(R R=1.02,95 \%$ $\mathrm{Crl}=0.92-1.13, \tau=0.18)$ but favoured there being no important association (7\% probability of RR $\geq 1.1)$. Former compared with never smokers were at somewhat increased risk of hospitalisation $(R R=1.19, \mathrm{Crl}=1.03-1.43, \tau=0.17)$, greater disease severity $(R R=1.52, \mathrm{Crl}=1.12-2.05, \tau=0.29)$ and mortality $(R R=1.35,95 \% \mathrm{Crl}=1.09-1.73, \tau=0.26)$. Data for current smokers on hospitalisation, disease severity and mortality were inconclusive $(R R=1.06, C r l=0.82-1.35$, 
$\tau=0.27 ; \mathrm{RR}=1.26, \mathrm{Crl}=0.85-1.96, \tau=0.34 ; \mathrm{RR}=1.10,95 \% \mathrm{Crl}=0.69-1.67, \tau=0.50$, respectively) but favoured there being no important associations with hospitalisation and mortality ( $35 \%$ and $51 \%$ probability of $R R \geq 1.1$, respectively) and a small but important association with disease severity $(79 \%$ probability of $R R \geq 1.1)$.

Conclusions: Compared with never smokers, current smokers appear to be at reduced risk of SARS-CoV-2 infection while former smokers appear to be at increased risk of hospitalisation, greater disease severity and mortality from COVID-19. However, it is uncertain whether these associations are causal.

\section{Introduction}

COVID-19 is a respiratory disease caused by the SARS-CoV-2 virus. Large age and gender differences in case severity and mortality have been observed in the ongoing COVID-19 pandemic ${ }^{1}$; however, these differences are currently unexplained. SARS-CoV-2 enters epithelial cells through the angiotensin-converting enzyme 2 (ACE-2) receptor ${ }^{2}$. Some evidence suggests that gene expression and subsequent receptor levels are elevated in the airway and oral epithelium of current smokers ${ }^{3,4}$, which could put smokers at higher risk of contracting SARS-CoV-2. Other studies, however, suggest that nicotine downregulates the ACE-2 receptor ${ }^{5}$. These uncertainties notwithstanding, both former and current smoking is known to increase the risk of respiratory viral ${ }^{6,7}$ and bacterial ${ }^{8,9}$ infections and is associated with worse outcomes once infected. Cigarette smoke reduces the respiratory immune defence through peri-bronchiolar inflammation and fibrosis, impaired mucociliary clearance and disruption of the respiratory epithelium ${ }^{10}$. There is also reason to believe that behavioural factors (e.g. regular hand-to-mouth movements) involved in smoking may increase SARS-CoV-2 infection and transmission in current smokers. However, early data from the COVID-19 pandemic have not provided clear evidence for a negative impact of current or former smoking on SARS-CoV-2 infection or COVID-19 disease outcomes, such as hospitalisation or mortality ${ }^{11}$. It has also been hypothesised that nicotine might protect against a hyper-inflammatory response to SARS-CoV-2 infection, which may lead to adverse outcomes in patients with COVID-19 disease ${ }^{12}$.

There are several reviews that fall within the scope of smoking and COVID-1911,13-18. We aimed to produce a rapid synthesis of available evidence pertaining to the rates of infection, hospitalisation, disease severity and mortality from SARS-CoV-2/COVID-19 stratified by smoking status. Given the increasing availability of data on this topic, this is a living review with regular updates. As evidence accumulates, the review will be expanded to include studies reporting COVID-19 outcomes by alternative nicotine use (e.g., nicotine replacement therapy or e-cigarettes).

\section{Methods}

\section{Study design}

This is a living evidence review which is updated as new evidence becomes available ${ }^{19}$. We adopted recommended best practice for rapid evidence reviews, which involved limiting the search to main databases and having one reviewer extract 
the data and another verify ${ }^{20}$. This study was not pre-registered but evolved from a report written for a UK medical society ${ }^{21}$. The most recent (and all future) version(s) of this living review is available here (https://www.qeios.com/read/latest-UJR2AW). Version 7 of this living review has been published in a peer-reviewed journal $^{22}$. A completed Preferred Reporting Items for Systematic Reviews and Meta-Analyses (PRISMA) checklist is included in Supplementary file 1.

\section{Eligibility criteria}

Studies were included if they:

1. Were primary research studies using experimental (e.g. randomised controlled trial), quasi-experimental (e.g. pre- and post-test) or observational (e.g. case-control, retrospective cohort, prospective cohort) study designs;

2. Included adults aged $16+$ years;

3. Recorded as outcome i) results of a SARS-CoV-2 diagnostic test (including antibody assays), ii) clinical diagnosis of COVID-19, iii) hospitalisation with COVID-19, iv) severity of COVID-19 disease in those hospitalised or v) mortality from COVID-19;

4. Reported any of the outcomes of interest by self-reported or biochemically verified smoking status (e.g. current smoker, former smoker, never smoker) or current vaping and nicotine replacement therapy (NRT) use;

5. Were available in English;

6. Were published in a peer-reviewed journal, as a pre-print or a public health report by reputable bodies (e.g. governments, scientific societies).

\section{Search strategy}

The following terms were searched for in Ovid MEDLINE (2019-search date) as free text or Medical Subject Headings:

1. Tobacco Smoking/ or Smoking Cessation/ or Water Pipe Smoking/ or Smoking/or Smoking Pipes/ or Cigar Smoking/ or Smoking Prevention/ or Cigarette Smoking/ or smoking.mp. or Pipe Smoking/ or Smoking, Non-Tobacco Products/ or Smoking Water Pipes/

2. Nicotine/ or nicotine.mp. or Electronic Nicotine Delivery Systems/ or Nicotine Chewing Gum/

3. vaping.mp. or Vaping/

4. 1 or 2 or 3

5. Coronavirus/ or Severe Acute Respiratory Syndrome/ or Coronavirus Infections/ or covid.mp.

6. 4 and 5

The following terms were searched for in titles, abstracts and full texts in medRxiv (no time limitations): 
1. covid (this term captures both covid and SARS-CoV-2) AND smoking

2. covid AND nicotine

3. covid AND vaping

Additional articles/reports of interest were identified through mailing lists, Twitter, the International Severe Acute Respiratory and Emerging Infection Consortium (ISARIC) and the US Centers for Disease Control and Prevention (CDC). Where updated versions of pre-prints or public health reports were available, old versions were superseded.

\section{Selection of studies}

One reviewer screened titles, abstracts and full texts against the inclusion criteria.

\section{Data extraction}

Data were extracted by one reviewer and verified (i.e. independently checked against pre-prints and published reports) by another on i) author (year); ii) date published; iii) country; iv) study design; v) study setting; vi) sample size; vii) sex; viii) age; ix) smoking status (e.g. current, former, never, not stated, missing) and whether it was biochemically verified; $x$ ) use of alternative nicotine products; xi) SARS-CoV-2 testing; xii) SARS-CoV-2 infection; xiii) diagnosis of COVID-19; xiv) hospitalisation with COVID-19; xv) disease severity in those hospitalised with COVID-19; xvi) mortality; xvii) adjustment of smoking specific risk estimates for relevant covariates (e.g. age, sex); and xviii) whether a representative or random sampling method was used.

\section{Quality appraisal}

The quality of included studies was assessed to determine suitability for inclusion in meta-analyses. Studies were judged as 'good' quality if they: i) had $<20 \%$ missing data on smoking status and used a reliable self-report measure that distinguished between current, former and never smoking status; AND ii) used biochemical verification of smoking status and reported results from adjusted analyses; OR reported data from a representative/random sample. Studies were rated as 'fair' if they fulfilled only criterion i) and were otherwise rated as 'poor'. The quality appraisal was conducted by one reviewer and verified by a second.

\section{Evidence synthesis}

A narrative synthesis was conducted. Data from 'good' and 'fair' quality studies were pooled in R v.3.6.3 ${ }^{23}$. In a living review where new data are regularly added to the analyses, it may be more appropriate to use a Bayesian (as opposed to frequentist) approach where prior knowledge is used in combination with new data to estimate a posterior risk distribution. A Bayesian approach mitigates the issue of performing multiple statistical tests, which can inflate family-wise error. A 
series of random-effects hierarchical Bayesian meta-analyses were performed with the $b r m s^{24}$ package to estimate the relative risk for each comparison with accompanying 95\% credible intervals (Crls). We first defined prior distributions for the true pooled effect size $(\mu)$ and the between-study heterogeneity $(\tau)$, with $\mu$ specified as a normal distribution with a mean equal to the derived point estimate from each comparison of interest in the immediately preceding version of this living review ${ }^{25}$, and $\tau$ specified as a half-Cauchy distribution with a mean of 0 and standard deviation of 1 . The halfCauchy distribution was selected to reflect prior knowledge that high levels of between-study heterogeneity are more likely than lower levels. Markov Chain Monte Carlo methods (20,000 burn-ins followed by 80,000 iterations) were then used to generate a risk distribution for each study, in addition to a pooled effect for the posterior risk distribution. We report forest plots with the pooled effect for the posterior risk distribution displayed as the median relative risk with an accompanying $95 \%$ Crls. We used the empirical cumulative distribution function (ECDF) to estimate the probability of there being a $10 \%$ reduction or $10 \%$ increase in relative risk ( $R R$ ) (i.e. $R R \geq 1.1$ or $R R \leq 0.9$ ). Due to a lack of indication as to what constitutes a clinically or epidemiologically meaningful effect (e.g. with regards to onward disease transmission or requirements for intensive care beds), we deemed a 10\% change in risk as small but important. Where data were inconclusive (as indicated by Crls crossing $R R=1.0$ ), to disambiguate whether data favoured no effect or there being a small but important association, we estimated whether there was $\geq 75 \%$ probability of $R R \geq 1.1$ or $R R \leq 0.9$.

Two sensitivity analyses were performed. First, a minimally informative prior for $\mu$ was specified as a normal distribution with a mean of 0 and standard deviation of 1 and $\tau$ as described above. Second, an informative prior as described above for $\mu$ was used with $\tau$ specified as a half-Cauchy distribution with a mean of 0.3 and standard deviation of 1 to reflect greater between-study heterogeneity.

To aid in the visualisation of smoking prevalence in the included studies, the weighted mean prevalence of current and former smoking was calculated for countries in which $\geq 3$ studies were conducted and plotted for comparison with national prevalence estimates. It should be noted that prevalence estimates in the included studies were not adjusted for age, sex, socioeconomic position, or region within countries.

\section{Results}

In the current review version (v8) with searches up to 22 September 2020, a total of 593 new records were identified, with 256 studies included in a narrative synthesis and 36 studies included in meta-analyses (see Figure 1). 


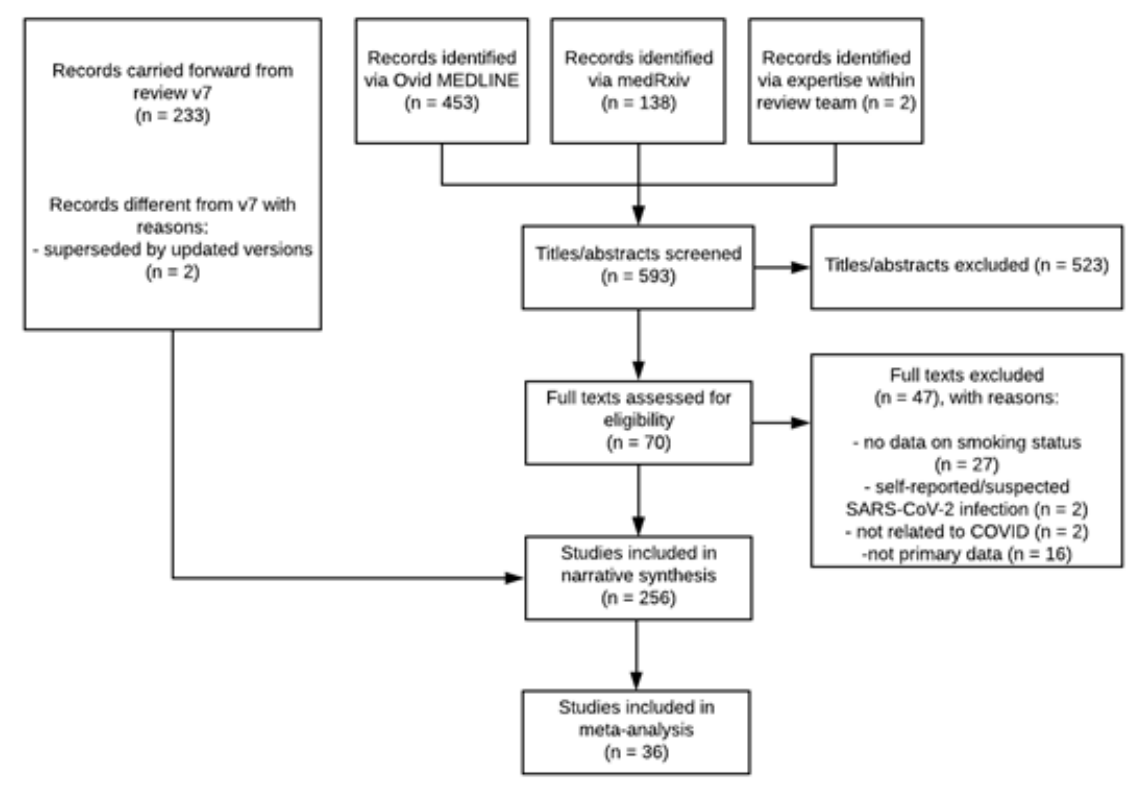

Figure 1. PRISMA flow diagram of included studies.

\section{Study characteristics}

Characteristics of included studies are presented in Table 1. Studies were conducted across 34 countries. Sixty-eight studies were conducted in the US, 56 in China, 30 in the UK, 15 in Spain, 14 in Mexico, 12 in France, seven in Italy, seven across multiple international sites, five in Iran, four in Brazil, three in Israel and Turkey, two in Australia, Bangladesh, Chile, Denmark, Finland, India, Japan and Qatar and one from 15 further countries (see Supplementary figure S1). The majority of studies used observational designs (see Supplementary table S1). One-hundred-and-sixty-five studies (64.5\%) were conducted in hospital settings, 73 studies (28.5\%) included a community component in addition to hospitalised patients and 16 studies (6.2\%) were conducted exclusively in the community, one in a quarantine centre and one did not state the study setting. Studies had a median of 428 (interquartile range $=129-1,765)$ participants. The majority of studies $(92.6 \%)$ used reverse transcriptase polymerase chain reaction (RT-PCR) for confirmation of SARS-CoV-2 infection, 3.5\% used an antibody test to confirm prior infection, and 3.9\% further studies relied on a combination of RT-PCR and clinical diagnosis (see Supplementary table S1).

\section{Smoking status}

Categorisation of smoking status was heterogeneous (see Table 1). One-hundred-and-fifty-five studies collected data on smoking status through routine electronic health records (EHRs), 72 studies used a bespoke case report form for COVID19 and 29 studies did not state the source for information on smoking status. None of the studies verified smoking status biochemically. Notably, only 67 (26.2\%) studies reported current, former and never smoking status (see Supplementary table S2a), with a further 18 studies reporting ever and never smoking status (see Supplementary table S2b). The 
remaining 171 studies reported current, current/former or current and former smoking status but did not explicitly state whether remaining participants were never smokers or if data were missing on smoking status (see Supplementary table S2c). Eighty-three studies explicitly reported the proportion with missing data on smoking status, which ranged from $0 \%$ to $96.4 \%$.

\section{Use of alternative nicotine products}

Five studies recorded the use of alternative nicotine products in current and/or former smokers but did not report COVID-19 outcomes stratified by nicotine use $26-30$.

\section{Quality appraisal}

Two studies were performed in random, representative population samples and were rated as 'good' quality. Fifty-two studies were rated as 'fair' quality, of which 36 studies reported results stratified by smoking status for the outcomes of interest and could be included in meta-analyses. The remaining 202 studies were rated as 'poor' quality (see Table 1).

Table 1. Characteristics of included studies.

\begin{tabular}{|c|c|c|c|c|c|c|c|c|c|c|c|c|}
\hline Ref. & Lead author & $\begin{array}{l}\text { Date } \\
\text { published }\end{array}$ & Country & $\begin{array}{l}\text { Sample } \\
\text { size }\end{array}$ & $\begin{array}{l}\text { Study } \\
\text { setting }\end{array}$ & $\begin{array}{l}\text { Median } \\
\text { (IQR) }\end{array}$ & $\begin{array}{l}\text { Female } \\
\%\end{array}$ & $\begin{array}{l}\text { Current } \\
\text { smoker } \\
\%\end{array}$ & $\begin{array}{l}\text { Former } \\
\text { smokers } \\
\%\end{array}$ & $\begin{array}{l}\text { Current/former } \\
\text { smokers \% }\end{array}$ & $\begin{array}{l}\text { Never } \\
\text { smokers } \\
\%\end{array}$ & $\begin{array}{l}\text { Never/unknowI } \\
\text { smokers \% }\end{array}$ \\
\hline 1 & Guan, Ni & $\begin{array}{l}2020-02- \\
28\end{array}$ & China & 1,099 & Hospital & $\begin{array}{c}47 \\
(35-58)\end{array}$ & 41.9 & 12.5 & 1.9 & - & 84.3 & - \\
\hline 55 & Guan, Liang & $\begin{array}{l}2020-03- \\
26\end{array}$ & China & 1,590 & Hospital & $\begin{array}{c}49 \\
(33-64)\end{array}$ & 42.7 & - & - & 7.0 & 93.0 & - \\
\hline 56 & Lian & $\begin{array}{l}2020-03- \\
25\end{array}$ & China & 788 & Hospital & NA & 38.5 & 6.9 & - & - & - & - \\
\hline 57 & Jin & $\begin{array}{l}2020-03- \\
24\end{array}$ & China & 651 & Hospital & $\begin{array}{c}46 \\
(32-60)\end{array}$ & 49.2 & 6.3 & - & - & - & - \\
\hline 58 & Chen & $\begin{array}{l}2020-03- \\
26\end{array}$ & China & 548 & Hospital & $\begin{array}{c}62 \\
(44-70)\end{array}$ & 37.6 & 4.4 & 2.6 & - & - & - \\
\hline 59 & Zhou, Yu & $\begin{array}{l}2020-03- \\
11\end{array}$ & China & 191 & Hospital & $\begin{array}{c}56 \\
(46-67)\end{array}$ & 38.0 & 5.8 & - & - & - & - \\
\hline 60 & Mo & $\begin{array}{l}2020-03- \\
16\end{array}$ & China & 155 & Hospital & $\begin{array}{c}54 \\
(53-66)\end{array}$ & 44.5 & 3.9 & - & - & - & - \\
\hline 61 & Zhang, Dong & $\begin{array}{l}2020-02- \\
19\end{array}$ & China & 140 & Hospital & $\begin{array}{c}57^{\wedge} \\
(25-87)\end{array}$ & 46.3 & 1.4 & 5.0 & - & - & - \\
\hline 62 & Wan & $\begin{array}{l}2020-03- \\
21\end{array}$ & China & 135 & Hospital & $\begin{array}{c}47 \\
(36-55)\end{array}$ & 46.7 & 6.7 & - & - & - & - \\
\hline 63 & Liu, Tao & $\begin{array}{l}2020-02- \\
28\end{array}$ & China & 78 & Hospital & $\begin{array}{c}38 \\
(33-57)\end{array}$ & 50.0 & - & - & 6.4 & - & - \\
\hline 64 & Huang, Wang & $\begin{array}{l}2020-01- \\
24\end{array}$ & China & 41 & Hospital & $\begin{array}{c}49 \\
(41-58)\end{array}$ & 27.0 & 7.3 & - & - & - & - \\
\hline 65 & Zhang, Cai & $\begin{array}{l}2020-03- \\
20\end{array}$ & China & 645 & Hospital & NA & 49.1 & 6.4 & - & - & - & - \\
\hline
\end{tabular}




\begin{tabular}{|c|c|c|c|c|c|c|c|c|c|c|c|}
\hline 66 & Guo & $\begin{array}{l}2020-03- \\
27\end{array}$ & China & 187 & Hospital & $\begin{array}{c}59 \\
(45-73)\end{array}$ & 51.3 & 9.6 & - & - & - \\
\hline 67 & Liu, Ming & $\begin{array}{l}2020-03- \\
12\end{array}$ & China & 41 & Hospital & $\begin{array}{c}39 \\
(30-48)\end{array}$ & 58.5 & 9.8 & - & - & - \\
\hline 68 & Huang, Yang & $\begin{array}{l}2020-03- \\
05\end{array}$ & China & 36 & Hospital & $\begin{array}{c}69 \\
(60-78)\end{array}$ & 30.6 & - & - & 11.1 & - \\
\hline 69 & $\mathrm{Xu}$ & $\begin{array}{l}2020-03- \\
08\end{array}$ & China & 53 & Hospital & NA & 47.2 & 11.3 & - & - & - \\
\hline 70 & $\mathrm{Li}$ & $\begin{array}{l}2020-02- \\
12\end{array}$ & China & 17 & Hospital & $\begin{array}{c}45 \\
(33-57)\end{array}$ & 47.1 & 17.6 & - & - & - \\
\hline 31 & Rentsch & $\begin{array}{l}2020-04- \\
14\end{array}$ & USA & 3,528 & $\begin{array}{l}\text { Community } \\
\text { and } \\
\text { Hospital }\end{array}$ & $\begin{array}{c}66 \\
(60-70)\end{array}$ & 4.6 & 27.2 & 30.6 & - & 36.9 \\
\hline 71 & $\mathrm{Hu}$ & $\begin{array}{l}2020-03- \\
25\end{array}$ & China & 323 & Hospital & $\begin{array}{c}61^{\wedge} \\
(23-91)\end{array}$ & 48.6 & - & - & 11.8 & - \\
\hline 72 & Wang, Pan & $\begin{array}{l}2020-03- \\
24\end{array}$ & China & 125 & Hospital & $\begin{array}{c}41 \\
(26-66)\end{array}$ & 43.2 & - & - & 12.8 & - \\
\hline 73 & $\begin{array}{l}\text { Chow (US } \\
\text { CDC) }\end{array}$ & $\begin{array}{l}2020-03- \\
31\end{array}$ & USA & 7,162 & $\begin{array}{l}\text { Community } \\
\text { and } \\
\text { Hospital }\end{array}$ & NA & - & 1.3 & 2.3 & - & - \\
\hline 74 & Dong, Cao & $\begin{array}{l}2020-03- \\
20\end{array}$ & China & 9 & Hospital & $\begin{array}{c}44 \\
(30-46)\end{array}$ & 66.7 & 11.1 & - & - & - \\
\hline 75 & Kim & $\begin{array}{l}2020-04- \\
01\end{array}$ & $\begin{array}{l}\text { South } \\
\text { Korea }\end{array}$ & 28 & Hospital & $\begin{array}{c}43 \\
(30-56)\end{array}$ & 46.4 & 17.9 & - & - & - \\
\hline 76 & Shi, Yu & $\begin{array}{l}2020-03- \\
18\end{array}$ & China & 487 & Hospital & $\begin{array}{c}46 \\
(27-65)\end{array}$ & 46.8 & - & - & 8.2 & - \\
\hline 77 & Yang, Yu & $\begin{array}{l}2020-02- \\
24\end{array}$ & China & 52 & Hospital & $\begin{array}{c}60 \\
(47-73)\end{array}$ & 37.0 & 3.8 & - & - & - \\
\hline 78 & Argenziano & $\begin{array}{l}2020-05- \\
29\end{array}$ & USA & 1,000 & Hospital & $\begin{array}{c}63 \\
(50-75)\end{array}$ & 40.4 & 4.9 & 17.9 & - & 77.2 \\
\hline 79 & Solis & $\begin{array}{l}2020-04- \\
25\end{array}$ & Mexico & 650 & Hospital & ${ }^{4}{ }^{46}$ & 42.1 & 9.4 & - & - & - \\
\hline 80 & Richardson & $\begin{array}{l}2020-04- \\
22\end{array}$ & USA & 5,700 & Hospital & $\begin{array}{c}63 \\
(52-75)\end{array}$ & 39.7 & - & - & 9.8 & 52.8 \\
\hline 81 & Fontanet & $\begin{array}{l}2020-04- \\
23\end{array}$ & France & 661 & $\begin{array}{l}\text { Community } \\
\text { and } \\
\text { Hospital }\end{array}$ & $\begin{array}{c}37 \\
(16-47)\end{array}$ & 62.0 & 10.4 & - & - & - \\
\hline 82 & Zheng, Gao & $\begin{array}{l}2020-04- \\
19\end{array}$ & China & 66 & Hospital & $\begin{array}{l}47^{\wedge} \\
(\mathrm{NA})\end{array}$ & 25.8 & 12.1 & - & - & - \\
\hline 83 & Liao, Feng & $\begin{array}{l}2020-04- \\
24\end{array}$ & China & 1,848 & Hospital & $\begin{array}{c}55 \\
(48-61)\end{array}$ & 54.7 & - & - & 0.4 & - \\
\hline 84 & Gil-Agudo & $\begin{array}{l}2020-04- \\
24\end{array}$ & Spain & 7 & Hospital & $\begin{array}{c}68 \\
(34-75)\end{array}$ & 28.6 & - & - & 42.9 & 57.1 \\
\hline 85 & Shi, Ren & $\begin{array}{l}2020-04- \\
23\end{array}$ & China & 134 & Hospital & $\begin{array}{c}46 \\
(34-58)\end{array}$ & 51.5 & - & - & 10.4 & - \\
\hline 86 & Hadjadj & $\begin{array}{l}2020-04- \\
23\end{array}$ & France & 50 & Hospital & $\begin{array}{c}55 \\
(50-63)\end{array}$ & 22.0 & 2.0 & 18.0 & - & 80.0 \\
\hline 87 & Gold (US CDC) & $\begin{array}{l}2020-04- \\
20\end{array}$ & USA & 305 & Hospital & NA & 50.5 & 5.2 & - & - & - \\
\hline 88 & Yu, Cai & $\begin{array}{l}2020-04- \\
27\end{array}$ & China & 95 & Hospital & NA & 44.2 & 8.4 & - & - & - \\
\hline
\end{tabular}




\begin{tabular}{|c|c|c|c|c|c|c|c|c|c|c|c|c|}
\hline 89 & Zheng, Xiong & $\begin{array}{l}2020-04- \\
30\end{array}$ & China & 73 & Hospital & $\begin{array}{l}43^{\wedge} \\
(\mathrm{NA})\end{array}$ & 45.2 & - & - & 11.0 & 89.0 & - \\
\hline 90 & de la Rica & $\begin{array}{l}2020-05- \\
11\end{array}$ & Spain & 48 & Hospital & $\begin{array}{c}66^{\wedge} \\
(33-88)\end{array}$ & 33.0 & - & - & 20.8 & - & - \\
\hline 91 & Yin, Yang & $\begin{array}{l}2020-05- \\
10\end{array}$ & China & 106 & Hospital & $\begin{array}{c}73 \\
(61-85)\end{array}$ & 39.6 & - & - & 17.0 & - & - \\
\hline 92 & Shi, Zuo & $\begin{array}{l}2020-05- \\
17\end{array}$ & USA & 172 & Hospital & $\begin{array}{c}63^{\wedge} \\
(44-82)\end{array}$ & 44.0 & - & - & 26.2 & - & - \\
\hline 93 & Cho & $\begin{array}{l}2020-05- \\
11\end{array}$ & UK & 322,341 & $\begin{array}{l}\text { Community } \\
\text { and } \\
\text { Hospital }\end{array}$ & NA & 49.2 & 14.2 & 21.4 & - & 64.4 & - \\
\hline 94 & Allenbach & $\begin{array}{l}2020-05- \\
08\end{array}$ & France & 152 & Hospital & $\begin{array}{c}77 \\
(60-83)\end{array}$ & 31.1 & - & - & 6.6 & - & - \\
\hline 95 & Robilotti & $\begin{array}{l}2020-05- \\
08\end{array}$ & USA & 423 & Hospital & NA & 50.0 & 2.1 & 37.6 & - & 58.6 & - \\
\hline 96 & $\begin{array}{l}\text { The } \\
\text { Opensafely } \\
\text { Collaborative }\end{array}$ & $\begin{array}{l}2020-07- \\
01\end{array}$ & UK & 17,278,392 & $\begin{array}{l}\text { Community } \\
\text { and } \\
\text { Hospital }\end{array}$ & NA & 50.1 & 17.0 & 32.9 & - & 45.9 & - \\
\hline 97 & Borobia & $\begin{array}{l}2020-05- \\
06\end{array}$ & Spain & 2,226 & Hospital & $\begin{array}{c}61 \\
(46-78)\end{array}$ & 52.0 & 7.1 & - & - & - & - \\
\hline 98 & Giacomelli & $\begin{array}{l}2020-05- \\
06\end{array}$ & Italy & 233 & Hospital & $\begin{array}{c}61 \\
(50-72)\end{array}$ & 31.9 & - & - & 30.0 & 70.0 & - \\
\hline 99 & Shah & $\begin{array}{l}2020-05- \\
06\end{array}$ & USA & 316 & Hospital & $\begin{array}{c}63 \\
(43-72)\end{array}$ & 48.1 & 16.5 & 17.7 & - & 42.1 & - \\
\hline 100 & Kolin & $\begin{array}{l}2020-05- \\
05\end{array}$ & UK & 502,536 & $\begin{array}{l}\text { Community } \\
\text { and } \\
\text { Hospital }\end{array}$ & $\begin{array}{c}56.5 \\
(48-64)\end{array}$ & 54.4 & 10.5 & 34.4 & - & 54.4 & - \\
\hline 101 & Lubetzky & $\begin{array}{l}2020-05- \\
08\end{array}$ & USA & 54 & Hospital & $\begin{array}{c}57 \\
(29-83)\end{array}$ & 62.0 & - & - & 22.2 & - & - \\
\hline 102 & Goyal & $\begin{array}{l}2020-04- \\
17\end{array}$ & USA & 393 & Hospital & $\begin{array}{c}62.2 \\
(49-74)\end{array}$ & 39.3 & 5.1 & - & - & - & - \\
\hline 103 & Feng & $\begin{array}{l}2020-04- \\
10\end{array}$ & China & 476 & Hospital & $\begin{array}{c}53 \\
(40-64)\end{array}$ & 43.1 & 9.2 & - & - & - & - \\
\hline 104 & Yao & $\begin{array}{l}2020-04- \\
24\end{array}$ & China & 108 & Hospital & $\begin{array}{c}52 \\
(37-58)\end{array}$ & 60.2 & 3.7 & - & - & - & - \\
\hline 105 & Sami & $\begin{array}{l}2020-05- \\
19\end{array}$ & Iran & 490 & Hospital & $\begin{array}{c}56.6 \\
(41-71)\end{array}$ & 39.0 & 14.1 & - & - & - & 85.9 \\
\hline 106 & Almazeedi & $\begin{array}{l}2020-05- \\
15\end{array}$ & Kuwait & 1,096 & Hospital & $\begin{array}{c}41 \\
(25-57)\end{array}$ & 19.0 & 4.0 & - & - & - & 96.0 \\
\hline 107 & Carillo-Vega & $\begin{array}{l}2020-05- \\
14\end{array}$ & Mexico & 10,544 & $\begin{array}{l}\text { Community } \\
\text { and } \\
\text { Hospital }\end{array}$ & $\begin{array}{c}46.5^{\wedge} \\
(30-62)\end{array}$ & 42.3 & 8.9 & - & - & - & - \\
\hline 108 & Yanover & $\begin{array}{l}2020-05- \\
13\end{array}$ & Israel & 4,353 & $\begin{array}{l}\text { Community } \\
\text { and } \\
\text { Hospital }\end{array}$ & $\begin{array}{c}35 \\
(22-54)\end{array}$ & 44.5 & 11.8 & 3.0 & - & 85.2 & - \\
\hline 109 & Hamer & $\begin{array}{l}2020-05- \\
13\end{array}$ & UK & 387,109 & Hospital & $\begin{array}{c}56.2 \\
(48-64)\end{array}$ & 55.1 & 9.7 & 34.8 & - & 55.5 & - \\
\hline 110 & Regina & $\begin{array}{l}2020-05- \\
14\end{array}$ & Switzerland & 200 & Hospital & $\begin{array}{c}70 \\
(55-81)\end{array}$ & 40.0 & 4.5 & - & - & - & - \\
\hline 111 & de Lusignan & $\begin{array}{l}2020-05- \\
. .\end{array}$ & UK & 3,802 & $\begin{array}{l}\text { Community } \\
\text { and }\end{array}$ & 58 & 57.6 & 10.9 & 46.1 & - & 29.6 & - \\
\hline
\end{tabular}




\begin{tabular}{|c|c|c|c|c|c|c|c|c|c|c|c|}
\hline & 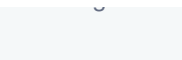 & 15 & & & Hospital & $(34-73)$ & & & & & \\
\hline 112 & Targher & $\begin{array}{l}2020-05- \\
13\end{array}$ & China & 339 & Hospital & $\begin{array}{l}48.4^{\wedge} \\
(\mathrm{NA})\end{array}$ & 52.8 & 8.3 & - & - & - \\
\hline 113 & Valenti & $\begin{array}{l}2020-05- \\
18\end{array}$ & Italy & 789 & Community & $\begin{array}{l}40.7^{\wedge} \\
\text { (NA) }\end{array}$ & 35.0 & 25.9 & - & - & - \\
\hline 114 & Feuth & $\begin{array}{l}2020-05- \\
18\end{array}$ & Finland & 28 & Hospital & $\begin{array}{c}56 \\
(47-72)\end{array}$ & 46.0 & 10.7 & 28.6 & - & 60.7 \\
\hline 115 & $\mathrm{Ge}$ & $\begin{array}{l}2020-05- \\
18\end{array}$ & China & 51 & Hospital & $\begin{array}{c}70 \\
(58-79)\end{array}$ & 27.5 & 13.7 & - & - & - \\
\hline 116 & Parrotta & $\begin{array}{l}2020-05- \\
18\end{array}$ & USA & 76 & $\begin{array}{l}\text { Community } \\
\text { and } \\
\text { Hospital }\end{array}$ & $\begin{array}{r}44.9 \\
(13-71)\end{array}$ & 61.8 & 2.6 & 26.3 & - & 68.4 \\
\hline 117 & Shekhar & $\begin{array}{l}2020-05- \\
18\end{array}$ & USA & 50 & Hospital & $\begin{array}{c}55.5 \\
(20-85)\end{array}$ & 54.0 & 48.0 & - & - & - \\
\hline 118 & Mejia-Vilet & $\begin{array}{l}2020-05- \\
16\end{array}$ & Mexico & 329 & Hospital & $\begin{array}{c}49 \\
(41-60)\end{array}$ & 36.0 & - & - & 7.0 & - \\
\hline 119 & Chen, Jiang & $\begin{array}{l}2020-05- \\
16\end{array}$ & China & 135 & Hospital & NA & 42.2 & - & - & 9.6 & - \\
\hline 120 & Li, Chen & $\begin{array}{l}2020-05- \\
16\end{array}$ & China & 1,008 & Hospital & $\begin{array}{c}55 \\
(44-65)\end{array}$ & 43.6 & 5.7 & - & - & - \\
\hline 27 & Rimland & $\begin{array}{l}2020-05- \\
19\end{array}$ & USA & 11 & Hospital & $\begin{array}{c}59 \\
(48-65)\end{array}$ & 18.2 & 9.1 & - & - & - \\
\hline 121 & Palaiodimos & $\begin{array}{l}2020-05- \\
15\end{array}$ & USA & 200 & Hospital & $\begin{array}{c}64 \\
(50- \\
73.5)\end{array}$ & 51.0 & - & - & 32.5 & 67.5 \\
\hline 122 & Ip & $\begin{array}{l}2020-05- \\
25\end{array}$ & USA & 2,512 & Hospital & $\begin{array}{c}64 \\
(52-76)\end{array}$ & 37.6 & 3.1 & 17.8 & - & 64.5 \\
\hline 123 & Heili-Frades & $\begin{array}{l}2020-05- \\
25\end{array}$ & Spain & 4,712 & Hospital & $\begin{array}{c}62 \\
(47-77)\end{array}$ & 50.5 & 4.9 & 17.4 & - & - \\
\hline 124 & $\begin{array}{l}\text { Vaquero- } \\
\text { Roncero }\end{array}$ & $\begin{array}{l}2020-05- \\
24\end{array}$ & Spain & 146 & Hospital & $\begin{array}{c}66^{\wedge} \\
(59-72)\end{array}$ & 32.2 & - & - & 6.8 & - \\
\hline 125 & Kim, Garg & $\begin{array}{l}2020-05- \\
22\end{array}$ & USA & 2,491 & Hospital & $\begin{array}{c}62 \\
(50-75)\end{array}$ & 46.8 & 6.0 & 25.8 & - & - \\
\hline 126 & Wu & $\begin{array}{l}2020-05- \\
21\end{array}$ & Italy & 174 & Hospital & $\begin{array}{c}61.2^{\wedge} \\
(50-71)\end{array}$ & 30.5 & - & - & 33.3 & - \\
\hline 127 & Shi, Zhao & $\begin{array}{l}2020-05- \\
20\end{array}$ & China & 101 & Hospital & $\begin{array}{c}71 \\
(59-80)\end{array}$ & 40.6 & - & - & 5.0 & - \\
\hline 128 & Al-Hindawi & $\begin{array}{l}2020-05- \\
20\end{array}$ & UK & 31 & Hospital & $(\mathrm{NA})^{61}$ & 12.9 & 3.2 & 71.0 & - & 25.8 \\
\hline 129 & Basse & $\begin{array}{l}2020-05- \\
19\end{array}$ & France & 141 & Hospital & $\begin{array}{c}62 \\
(52-72)\end{array}$ & 72.0 & 17.7 & - & - & - \\
\hline 130 & Freites & $\begin{array}{l}2020-05- \\
19\end{array}$ & Spain & 123 & Hospital & $\begin{array}{l}59.88^{\wedge} \\
(44-74)\end{array}$ & 69.9 & 3.3 & - & - & - \\
\hline 131 & Alshami & $\begin{array}{l}2020-05- \\
19\end{array}$ & $\begin{array}{l}\text { Saudi } \\
\text { Arabia }\end{array}$ & 128 & $\begin{array}{l}\text { Quarantine } \\
\text { Centre }\end{array}$ & $\begin{array}{c}39.6^{\wedge} \\
(24-55)\end{array}$ & 53.9 & 15.6 & 2.3 & - & - \\
\hline 132 & Berumen & $\begin{array}{l}2020-05- \\
26\end{array}$ & Mexico & 102,875 & Hospital & NA & 49.1 & - & - & 9.6 & - \\
\hline 133 & Gianfrancesco & 2020-05- & Multiole & 600 & $\begin{array}{l}\text { Community } \\
\text { and }\end{array}$ & 56 & 71.0 & - & - & 21.5 & 64.8 \\
\hline
\end{tabular}




\begin{tabular}{|c|c|c|c|c|c|c|c|c|c|c|c|c|}
\hline & & 29 & & & Hospital & $(45-67)$ & & & & & & \\
\hline 134 & Li, Long & $\begin{array}{l}2020-05- \\
28\end{array}$ & China & 145 & Not Stated & $\begin{array}{c}49^{\wedge} \\
(13-80)\end{array}$ & 61.0 & - & - & 5.5 & - & - \\
\hline 135 & Batty & $\begin{array}{l}2020-06- \\
17\end{array}$ & UK & 908 & Hospital & $\begin{array}{l}57.27^{\wedge} \\
(48-66)\end{array}$ & 44.3 & 11.2 & - & - & - & - \\
\hline 136 & Israel & $\begin{array}{l}2020-06- \\
01\end{array}$ & Israel & 24,906 & $\begin{array}{l}\text { Community } \\
\text { and } \\
\text { Hospital }\end{array}$ & $\begin{array}{c}40 \\
(27-59)\end{array}$ & 48.7 & 16.8 & 12.7 & - & 70.5 & - \\
\hline 137 & del Valle & $\begin{array}{l}2020-05- \\
30\end{array}$ & USA & 1,484 & Hospital & $\begin{array}{c}62 \\
(52-72)\end{array}$ & 40.6 & 5.5 & 23.3 & - & - & - \\
\hline 138 & Chaudhry & $\begin{array}{l}2020-05- \\
29\end{array}$ & USA & 40 & $\begin{array}{l}\text { Community } \\
\text { and } \\
\text { Hospital }\end{array}$ & $\begin{array}{l}52 \\
(45.5- \\
61)\end{array}$ & 60.0 & - & - & 15.0 & - & - \\
\hline 139 & Louis & $\begin{array}{l}2020-05- \\
28\end{array}$ & USA & 22 & Hospital & $\begin{array}{r}66.5^{\wedge} \\
(55-77)\end{array}$ & 36.4 & - & - & 45.5 & - & - \\
\hline 140 & Soto-Mota & $\begin{array}{l}2020-06- \\
05\end{array}$ & Mexico & 400 & Hospital & NA & 30.0 & - & - & 12.0 & - & - \\
\hline 141 & Garibaldi & $\begin{array}{l}2020-05- \\
26\end{array}$ & USA & 832 & Hospital & $\begin{array}{c}63 \\
(49-75)\end{array}$ & 47.0 & 5.5 & 22.6 & - & - & - \\
\hline 142 & Docherty & $\begin{array}{l}2020-05- \\
22\end{array}$ & Multiple & 20,133 & Hospital & $\begin{array}{c}72.9 \\
(58-82)\end{array}$ & 40.0 & 4.2 & 21.7 & - & 44.5 & - \\
\hline 143 & Boulware & $\begin{array}{l}2020-06- \\
03\end{array}$ & Multiple & 821 & Community & $\begin{array}{c}40 \\
(33-50)\end{array}$ & 51.6 & 3.3 & - & - & - & - \\
\hline 144 & Kuderer & $\begin{array}{l}2020-05- \\
28\end{array}$ & Multiple & 928 & $\begin{array}{l}\text { Community } \\
\text { and } \\
\text { Hospital }\end{array}$ & $\begin{array}{c}66 \\
(57-76)\end{array}$ & 50.0 & 4.6 & 35.1 & - & 50.5 & - \\
\hline 145 & Romao & $\begin{array}{l}2020-06- \\
08\end{array}$ & Portugal & 34 & Community & $\begin{array}{c}41^{\wedge} \\
(26-66)\end{array}$ & 67.7 & - & - & 26.5 & - & - \\
\hline 146 & Giannouchos & $\begin{array}{l}2020-06- \\
07\end{array}$ & Mexico & 236,439 & $\begin{array}{l}\text { Community } \\
\text { and } \\
\text { Hospital }\end{array}$ & $\begin{array}{c}42.5^{\wedge} \\
(25-59)\end{array}$ & 49.1 & 9.1 & - & - & - & 90.9 \\
\hline 147 & Ramlall & $\begin{array}{l}2020-06- \\
06\end{array}$ & USA & 11,116 & $\begin{array}{l}\text { Community } \\
\text { and } \\
\text { Hospital }\end{array}$ & $\begin{array}{c}52 \\
(34.7- \\
69.5)\end{array}$ & 55.2 & - & - & 26.8 & 73.2 & - \\
\hline 148 & Wang, Oekelen & $\begin{array}{l}2020-06- \\
05\end{array}$ & USA & 58 & $\begin{array}{l}\text { Community } \\
\text { and } \\
\text { Hospital }\end{array}$ & $(\mathrm{NA})^{67}$ & 48.0 & - & - & 36.2 & - & - \\
\hline 149 & Perrone & $\begin{array}{l}2020-06- \\
05\end{array}$ & Italy & 1,189 & Hospital & NA & 21.2 & - & - & 21.9 & - & - \\
\hline 150 & Sharma & $\begin{array}{l}2020-06- \\
05\end{array}$ & India & 501 & Hospital & $\begin{array}{r}35.1^{\wedge} \\
(18-51)\end{array}$ & 36.0 & - & - & 4.2 & - & - \\
\hline 151 & Eugen-Olsen & $\begin{array}{l}2020-06- \\
02\end{array}$ & Denmark & 407 & Hospital & $\begin{array}{c}64 \\
(47-77)\end{array}$ & 57.7 & 20.6 & 36.9 & - & 39.6 & - \\
\hline 152 & $\begin{array}{l}\text { Martinez- } \\
\text { Portilla }\end{array}$ & $\begin{array}{l}2020-06- \\
02\end{array}$ & Mexico & 224 & $\begin{array}{l}\text { Community } \\
\text { and } \\
\text { Hospital }\end{array}$ & $\begin{array}{c}29 \\
(26-33)\end{array}$ & 100.0 & - & - & 3.1 & - & - \\
\hline 153 & $\begin{array}{l}\text { Raisi- } \\
\text { Estabragh }\end{array}$ & $\begin{array}{l}2020-06- \\
02\end{array}$ & UK & 4,510 & Hospital & NA & 48.8 & - & - & 51.8 & - & - \\
\hline 154 & Luo & $\begin{array}{l}2020-06- \\
02\end{array}$ & China & 625 & Hospital & ${ }{ }^{46}$ & 47.7 & 3.0 & - & - & - & - \\
\hline
\end{tabular}


09

156 Cen

157 Klang

158 Maraschini

159 Wang, Zhong

2020-06-

12

2020-06-

12

UK

428,199

2020-06-

12

France

2020-06-

12

UK

1,737

2020-06-

$$
11
$$

UK

4,510

163 Hultcrantz

2020-06-

11

164 Rajter

2020-06-

10

165 Lan

166 Zeng

167 Suleyman

68 Chen, Yu

169 Garassino

2020-06-

09

2020-06-

16

2020-06-

16

2020-06-

16

2020-06-

$12^{\text {Multiple } 200}$

70 HernandezGarduno

2020-06

11

Mexico

32,583

2020-06- UK

20

UK

6,309

172 Siso-Almirall

$2020-06$
20

Spain

$173 \mathrm{Gu}$

2020-06-

18

USA

5,698

174 Kibler

France

\begin{tabular}{l|l|l}
\hline $2020-06-$ & France & 702 \\
16 &
\end{tabular}

$(29-44)$

\begin{tabular}{|c|c|c|c|c|}
\hline Hospital & $\begin{array}{c}61 \\
(49-68)\end{array}$ & 51.0 & - & - \\
\hline Hospital & NA & 61.8 & - & - \\
\hline Hospital & $\begin{array}{r}32.5^{\wedge} \\
(27-38)\end{array}$ & 100.0 & - & 9.6 \\
\hline $\begin{array}{l}\text { Community } \\
\text { and } \\
\text { Hospital }\end{array}$ & NA & 45.1 & 3.6 & 17.1 \\
\hline ommunity & & & & \\
\hline
\end{tabular}

and

NA

54.9

44.4

80.8

Community

and

\begin{tabular}{l|l|l|l} 
NA & 44.7 & 6.7 & 31.6
\end{tabular}

Hospital

Hospital

$\begin{array}{ll}63.4^{\wedge} \quad 30.4 & -\end{array}$

(NA)

Community

\begin{tabular}{l|l|l|l|l|} 
and & 70.5 & 51.2 & 13.0 & 38.1
\end{tabular}

48.

Hospital (NA)

Community

and 68

Hospital

\begin{tabular}{c|c}
68 & 46.0 \\
$(41-91)$
\end{tabular}$\quad-$

72.4

\begin{tabular}{l|l} 
Hospital $\quad 59.6^{\wedge}$ \\
\hline
\end{tabular}

\begin{tabular}{|c|c|c|c}
\hline $59.6^{\wedge}$ & 45.5 & 5.7 & 10.7
\end{tabular}

74.6

Community $\begin{gathered}49^{\wedge} \\ (34-63)\end{gathered} \mid 47$.

Hospital $\quad 60.3^{\wedge}$

Hospital

(46-74)

47.8

Hospital

$57.5^{\wedge}$

$(40-74)$

$55.9-$

\begin{tabular}{l|c|c|c|c} 
Hospital & $\begin{array}{c}59 \\
(45-68)\end{array}$ & 50.0 & 2.4 & 3.6
\end{tabular}

Community 68

$\begin{array}{lllll}\text { and } & (61.8- & 30.0 & 24.0 & 55.5\end{array}$

Hospital 75)

Community

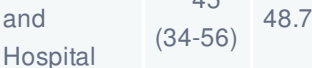

88.8

Community

and $46.5^{\wedge}$

Hospital

(31-61)

38.3

66.3

26.8

Community

and $56.7^{\wedge}$

Hospital

(38-74) 50.

Community

and $47^{\wedge}$

Hospital

\begin{tabular}{c|c|c}
$47^{\wedge}$ & 62.0 & 7.0
\end{tabular}

50.8

\begin{tabular}{l|c|c|c|c|}
\hline and & $82^{\wedge}$ & 56.0 & 3.7 & - \\
\hline Hospital & $(75-88)$ & & &
\end{tabular}
59.5

94.0

55.0

.5

โॅ八 


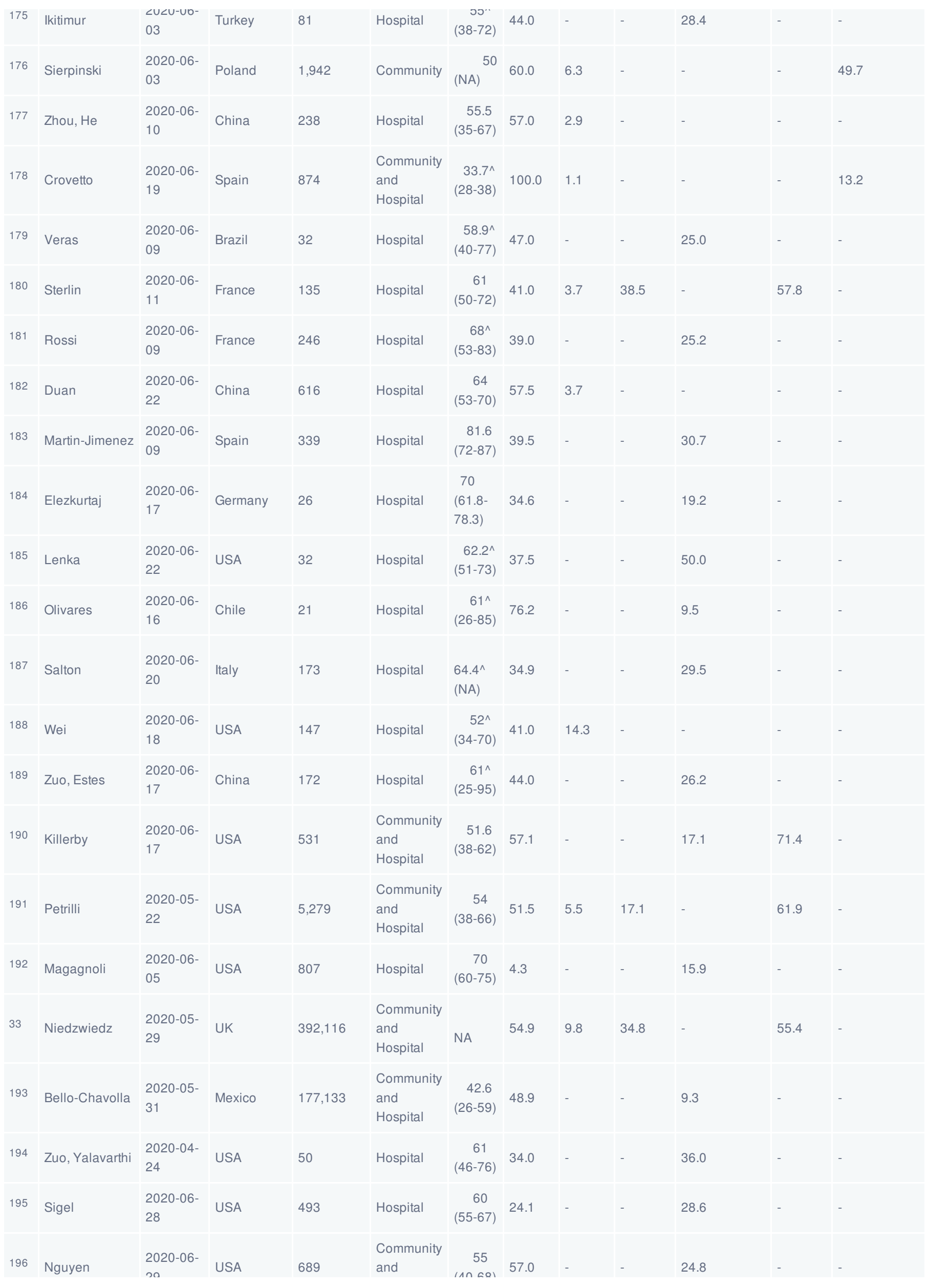




\begin{tabular}{|c|c|c|c|c|c|c|c|c|c|c|c|}
\hline & & $\angle \sigma$ & & & Hospital & $(+u-v 0)$ & & & & & \\
\hline 197 & de Melo & $\begin{array}{l}2020-06- \\
29\end{array}$ & Brazil & 181 & Hospital & $\begin{array}{r}55.3^{\wedge} \\
(34-76)\end{array}$ & 60.8 & 9.9 & 12.2 & - & 38.1 \\
\hline 198 & Auvinen & $\begin{array}{l}2020-06- \\
29\end{array}$ & Finland & 61 & Hospital & $\begin{array}{c}53 \\
(41-67)\end{array}$ & 36.0 & 18.0 & 27.9 & - & 54.1 \\
\hline 199 & Souza & $\begin{array}{l}2020-06- \\
28\end{array}$ & Brazil & 8,443 & Hospital & NA & 53.0 & - & - & 1.7 & - \\
\hline 200 & Mendy & $\begin{array}{l}2020-06- \\
27\end{array}$ & USA & 689 & $\begin{array}{l}\text { Community } \\
\text { and } \\
\text { Hospital }\end{array}$ & $\begin{array}{l}49.5 \\
(35.2- \\
67.5)\end{array}$ & 47.0 & - & - & 24.7 & - \\
\hline 201 & Pongpirul & $\begin{array}{l}2020-06- \\
26\end{array}$ & Thailand & 193 & Hospital & $\begin{array}{c}37 \\
(29-53)\end{array}$ & 41.5 & - & - & 15.0 & 66.3 \\
\hline 202 & Jin, Gu & $\begin{array}{l}2020-06- \\
25\end{array}$ & China & 6 & Hospital & $\begin{array}{r}60.5^{\wedge} \\
(51-75)\end{array}$ & 33.3 & 33.3 & - & - & - \\
\hline 203 & Favara & $\begin{array}{l}2020-05- \\
23\end{array}$ & UK & 70 & $\begin{array}{l}\text { Community } \\
\text { and } \\
\text { Hospital }\end{array}$ & $\begin{array}{c}41 \\
(23-64)\end{array}$ & 87.1 & 10.0 & - & - & - \\
\hline 204 & Fisman & $\begin{array}{l}2020-06- \\
23\end{array}$ & Canada & 21,922 & $\begin{array}{l}\text { Community } \\
\text { and } \\
\text { Hospital }\end{array}$ & NA & 57.0 & - & - & 2.3 & - \\
\hline 205 & Madariaga & $\begin{array}{l}2020-06- \\
23\end{array}$ & USA & 103 & $\begin{array}{l}\text { Community } \\
\text { and } \\
\text { Hospital }\end{array}$ & $\begin{array}{r}41.8^{\wedge} \\
(27-55)\end{array}$ & 48.5 & - & - & 25.2 & 74.8 \\
\hline 206 & Senkal & $\begin{array}{l}2020-07- \\
07\end{array}$ & Turkey & 611 & Hospital & $\begin{array}{c}57^{\wedge} \\
(18-98)\end{array}$ & 40.6 & 11.3 & - & - & - \\
\hline 207 & Mohamud & $\begin{array}{l}2020-07- \\
02\end{array}$ & USA & 6 & Hospital & $\begin{array}{r}65.8^{\wedge} \\
(55-78)\end{array}$ & 16.7 & - & - & 16.7 & - \\
\hline 208 & Magleby & $\begin{array}{l}2020-06- \\
30\end{array}$ & USA & 678 & Hospital & $\begin{array}{c}68 \\
(50-81)\end{array}$ & 38.9 & - & - & 28.6 & - \\
\hline 209 & Kimmig & $\begin{array}{l}2020-07- \\
06\end{array}$ & USA & 111 & Hospital & $\begin{array}{c}63^{\wedge} \\
(48-78)\end{array}$ & 44.1 & 7.2 & 36.0 & - & 56.8 \\
\hline 210 & $\begin{array}{l}\text { Bello-Chavolla, } \\
\text { Antonio-Villa }\end{array}$ & $\begin{array}{l}2020-07- \\
04\end{array}$ & Mexico & 60,121 & $\begin{array}{l}\text { Community } \\
\text { and } \\
\text { Hospital }\end{array}$ & $\begin{array}{c}45.5^{\wedge} \\
(29-61)\end{array}$ & 47.0 & - & - & 10.5 & - \\
\hline 211 & Zacharioudakis & $\begin{array}{l}2020-07- \\
04\end{array}$ & USA & 314 & Hospital & $\begin{array}{c}64 \\
(54-72)\end{array}$ & 34.7 & - & - & 22.8 & - \\
\hline 212 & Antonio-Villa & $\begin{array}{l}2020-07- \\
04\end{array}$ & Mexico & 34,263 & $\begin{array}{l}\text { Community } \\
\text { and } \\
\text { Hospital }\end{array}$ & $\begin{array}{c}40^{\wedge} \\
(29-50)\end{array}$ & 62.9 & 9.7 & - & - & - \\
\hline 213 & Patel & $\begin{array}{l}2020-07- \\
03\end{array}$ & USA & 129 & Hospital & $\begin{array}{r}60.8^{\wedge} \\
(47-74)\end{array}$ & 45.0 & 37.2 & - & - & - \\
\hline 214 & Merzon & $\begin{array}{l}2020-07- \\
03\end{array}$ & Israel & 7,807 & $\begin{array}{l}\text { Community } \\
\text { and } \\
\text { Hospital }\end{array}$ & $\begin{array}{l}46.2^{\wedge} \\
(\mathrm{NA})\end{array}$ & 58.6 & - & - & 16.2 & - \\
\hline 34 & Trubiano & $\begin{array}{l}2020-07- \\
02\end{array}$ & Australia & 2,935 & $\begin{array}{l}\text { Community } \\
\text { and } \\
\text { Hospital }\end{array}$ & $\begin{array}{c}39 \\
(29-53)\end{array}$ & 63.5 & - & - & 8.8 & - \\
\hline 215 & Fan & $\begin{array}{l}2020-07- \\
11\end{array}$ & UK & 1,425 & $\begin{array}{l}\text { Community } \\
\text { and } \\
\text { Hospital }\end{array}$ & NA & 46.7 & 12.2 & 40.1 & - & 46.9 \\
\hline 216 & $\begin{array}{l}\text { Shi, } \\
\text { Resurreccion }\end{array}$ & $\begin{array}{l}2020-07- \\
11\end{array}$ & UK & 1,521 & $\begin{array}{l}\text { Community } \\
\text { and } \\
\text { Hospital }\end{array}$ & $\begin{array}{l}61.5^{\wedge} \\
(57- \\
66.8)\end{array}$ & 45.9 & - & - & 54.9 & - \\
\hline
\end{tabular}




\begin{tabular}{|c|c|c|c|c|c|c|c|c|c|c|}
\hline 217 & Maucourant & $\begin{array}{l}2020-07- \\
10\end{array}$ & Sweden & 27 & Hospital & $\begin{array}{c}57 \\
(18-78)\end{array}$ & 22.2 & 11.1 & 25.9 & - \\
\hline 218 & Elmunzer & $\begin{array}{l}2020-07- \\
09\end{array}$ & Multiple & 1,992 & Hospital & $\begin{array}{c}60^{\wedge} \\
(43-76)\end{array}$ & 43.0 & 6.3 & 28.6 & - \\
\hline 219 & Alizadehsani & $\begin{array}{l}2020-07- \\
09\end{array}$ & Iran & 319 & Hospital & $\begin{array}{l}45.48^{\wedge} \\
(26-63)\end{array}$ & 55.5 & - & - & 0.3 \\
\hline 220 & Xie & $\begin{array}{l}2020-07- \\
07\end{array}$ & China & 619 & Hospital & NA & 52.0 & - & - & 8.2 \\
\hline 36 & Merkely & $\begin{array}{l}2020-07- \\
17\end{array}$ & Hungary & 10,474 & Community & $\begin{array}{r}48.7^{\wedge} \\
(30-66)\end{array}$ & 53.6 & 28.0 & 20.5 & - \\
\hline 221 & Fox & $\begin{array}{l}2020-07- \\
17\end{array}$ & UK & 55 & $\begin{array}{l}\text { Community } \\
\text { and } \\
\text { Hospital }\end{array}$ & $\begin{array}{c}63 \\
(23-88)\end{array}$ & 31.0 & 1.8 & 10.9 & - \\
\hline 61 & Zhang, Cao & $\begin{array}{l}2020-07- \\
14\end{array}$ & China & 289 & Hospital & $\begin{array}{c}57 \\
(22-88)\end{array}$ & 46.6 & 3.5 & 6.2 & - \\
\hline 222 & $\begin{array}{l}\text { Martinez-- } \\
\text { Resendez }\end{array}$ & $\begin{array}{l}2020-07- \\
20\end{array}$ & Mexico & 8 & Hospital & $\begin{array}{c}57 \\
(48-69)\end{array}$ & 25.0 & - & - & 12.5 \\
\hline 223 & Hoertel & $\begin{array}{l}2020-07- \\
20\end{array}$ & France & 12,612 & Hospital & $\begin{array}{r}58.7^{\wedge} \\
(39-77)\end{array}$ & 49.6 & - & - & 9.3 \\
\hline 224 & Mcgrail & $\begin{array}{l}2020-07- \\
19\end{array}$ & USA & 209 & Hospital & $\begin{array}{l}62.5 \\
\text { (NA) }\end{array}$ & 38.8 & - & - & 18.7 \\
\hline 225 & Pandolfi & $\begin{array}{l}2020-07- \\
17\end{array}$ & Italy & 33 & Hospital & $\begin{array}{c}62 \\
(52-65)\end{array}$ & 21.1 & 3.0 & 24.2 & - \\
\hline 28 & Girardeau & $\begin{array}{l}2020-07- \\
17\end{array}$ & France & 10 & Community & $\begin{array}{c}30 \\
(29-33)\end{array}$ & 50.0 & 40.0 & 10.0 & - \\
\hline 226 & Kurashima & $\begin{array}{l}2020-07- \\
17\end{array}$ & Japan & 53 & Hospital & $\begin{array}{r}62.9^{\wedge} \\
(49-76)\end{array}$ & 35.8 & - & - & 50.9 \\
\hline 227 & Zhan & $\begin{array}{l}2020-07- \\
16\end{array}$ & China & 75 & Hospital & $\begin{array}{c}57 \\
(25-75)\end{array}$ & 48.0 & - & - & 12.0 \\
\hline 228 & Omrani & $\begin{array}{l}2020-07- \\
16\end{array}$ & Qatar & 1,409 & $\begin{array}{l}\text { Community } \\
\text { and } \\
\text { Hospital }\end{array}$ & $\begin{array}{c}39 \\
(30-50)\end{array}$ & 17.2 & - & - & 9.2 \\
\hline 229 & Gupta & $\begin{array}{l}2020-07- \\
16\end{array}$ & USA & 496 & Hospital & $\begin{array}{c}70 \\
(60-78)\end{array}$ & 46.0 & - & - & 7.3 \\
\hline 92 & Shi, Zuo & $\begin{array}{l}2020-07- \\
15\end{array}$ & USA & 172 & Hospital & $\begin{array}{l}61.48^{\wedge} \\
(25-96)\end{array}$ & 44.0 & - & - & 26.2 \\
\hline 230 & Hussein & $\begin{array}{l}2020-07- \\
15\end{array}$ & USA & 502 & Hospital & $\begin{array}{r}60.9^{\wedge} \\
(45-76)\end{array}$ & 52.0 & 9.0 & 22.1 & - \\
\hline 231 & Bian & $\begin{array}{l}2020-07- \\
15\end{array}$ & China & 28 & Hospital & $\begin{array}{c}56^{\wedge} \\
(42-67)\end{array}$ & 42.9 & 7.1 & - & - \\
\hline 232 & Eiros & $\begin{array}{l}2020-07- \\
14\end{array}$ & Spain & 139 & $\begin{array}{l}\text { Community } \\
\text { and } \\
\text { Hospital }\end{array}$ & $\begin{array}{c}52 \\
(41-57)\end{array}$ & 72.0 & 4.3 & 50.4 & - \\
\hline 233 & Marcos & $\begin{array}{l}2020-07- \\
14\end{array}$ & Spain & 918 & Hospital & $\begin{array}{r}72.8^{\wedge} \\
(58-87)\end{array}$ & 42.2 & 6.1 & - & 15.3 \\
\hline 234 & $\begin{array}{l}\text { Hoertel, } \\
\text { Sanchez-Rico }\end{array}$ & $\begin{array}{l}2020-07- \\
14\end{array}$ & France & 7,345 & Hospital & NA & 49.3 & 8.5 & - & - \\
\hline 235 & Soares & $\begin{array}{l}2020-07- \\
16\end{array}$ & Brazil & 10,713 & $\begin{array}{l}\text { Community } \\
\text { and }\end{array}$ & NA & 55.0 & 2.0 & - & - \\
\hline
\end{tabular}




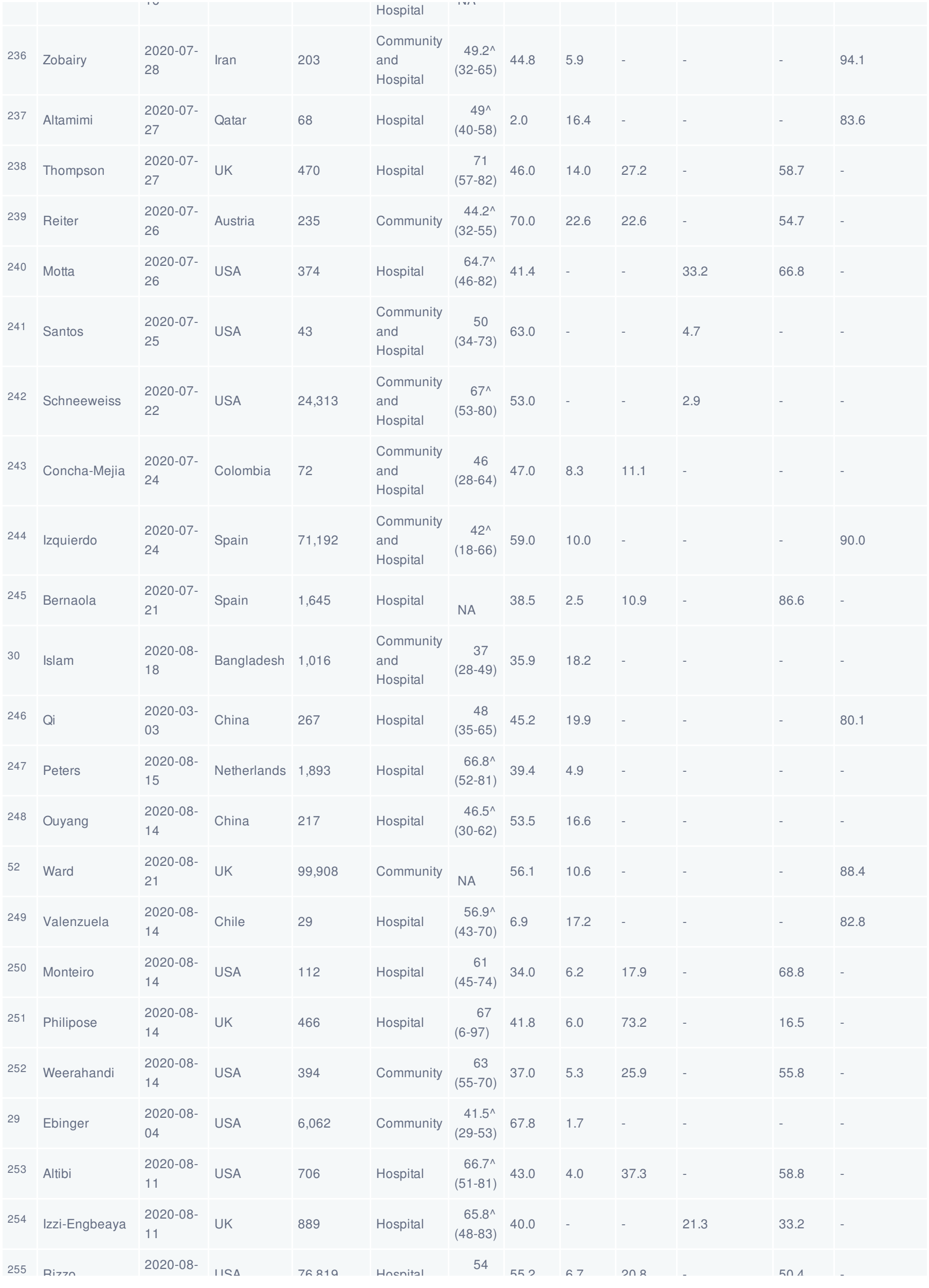




\begin{tabular}{|c|c|c|c|c|c|c|c|c|c|c|c|c|}
\hline & $\because u<<v$ & 11 & חיט & , ט, & & $(38-67)$ & uv.c & u., & cu.v & & טv.T & \\
\hline 256 & Dashti & $\begin{array}{l}2020-08- \\
04\end{array}$ & USA & 4,140 & $\begin{array}{l}\text { Community } \\
\text { and } \\
\text { Hospital }\end{array}$ & $\begin{array}{c}52 \\
(36-65)\end{array}$ & 55.0 & - & - & 28.4 & 51.6 & - \\
\hline 257 & Morshed & $\begin{array}{l}2020-08- \\
02\end{array}$ & Bangladesh & 103 & Community & $\begin{array}{c}37 \\
(31-53)\end{array}$ & 28.2 & 31.1 & - & - & - & 68.9 \\
\hline 258 & Jun & $\begin{array}{l}2020-08- \\
01\end{array}$ & USA & 3,086 & Hospital & $\begin{array}{c}66 \\
(56-77)\end{array}$ & 40.9 & 3.7 & 21.3 & - & 52.8 & - \\
\hline 259 & Higuchi & $\begin{array}{l}2020-07- \\
30\end{array}$ & Japan & 57 & Hospital & $\begin{array}{c}52 \\
(35-70)\end{array}$ & 43.9 & 12.3 & 29.8 & - & 57.9 & - \\
\hline 260 & Zhou, Sun & $\begin{array}{l}2020-07- \\
29\end{array}$ & China & 144 & Hospital & $\begin{array}{c}47 \\
(38-56)\end{array}$ & 46.5 & 9.0 & - & - & - & 91.0 \\
\hline 261 & Salerno & $\begin{array}{l}2020-08- \\
22\end{array}$ & USA & 15,920 & Hospital & $\begin{array}{c}49 \\
(30-65)\end{array}$ & 57.0 & - & - & 36.8 & 55.9 & - \\
\hline 262 & Kumar & $\begin{array}{l}2020-07- \\
29\end{array}$ & India & 91 & Hospital & $\begin{array}{c}47^{\wedge} \\
(41-52)\end{array}$ & 21.0 & 44.0 & - & - & - & - \\
\hline 263 & $\mathrm{Hao}$ & $\begin{array}{l}2020-06- \\
01\end{array}$ & China & 788 & Hospital & $\begin{array}{c}46 \\
(35-56)\end{array}$ & 48.4 & 6.9 & - & - & - & - \\
\hline 264 & Iversen & $\begin{array}{l}2020-08- \\
03\end{array}$ & Denmark & 28,792 & $\begin{array}{l}\text { Community } \\
\text { and } \\
\text { Hospital }\end{array}$ & $\begin{array}{r}44.4^{\wedge} \\
(31-57)\end{array}$ & 78.9 & 16.0 & 6.5 & - & 76.8 & - \\
\hline 265 & Hippisley-Cox & $\begin{array}{l}2020-07- \\
13\end{array}$ & UK & $8,275,949$ & $\begin{array}{l}\text { Community } \\
\text { and } \\
\text { Hospital }\end{array}$ & $\begin{array}{r}48.5^{\wedge} \\
(30-66)\end{array}$ & 50.3 & 17.2 & 21.4 & - & 57.3 & - \\
\hline 266 & Fillmore & $\begin{array}{l}2020-08- \\
24\end{array}$ & USA & 22,914 & $\begin{array}{l}\text { Community } \\
\text { and } \\
\text { Hospital }\end{array}$ & NA & - & 37.5 & 40.7 & - & 15.5 & - \\
\hline 267 & Rashid & $\begin{array}{l}2020-08- \\
22\end{array}$ & UK & 517 & Hospital & $\begin{array}{r}72.8^{\wedge} \\
(59-86)\end{array}$ & 31.9 & 9.9 & 29.0 & - & 29.4 & - \\
\hline 268 & Pan & $\begin{array}{l}2020-08- \\
22\end{array}$ & USA & 12,084 & $\begin{array}{l}\text { Community } \\
\text { and } \\
\text { Hospital }\end{array}$ & $\begin{array}{r}45.5^{\wedge} \\
(27-63)\end{array}$ & 54.3 & - & - & 17.5 & - & - \\
\hline 269 & Alkurt & $\begin{array}{l}2020-08- \\
20\end{array}$ & Turkey & 932 & $\begin{array}{l}\text { Community } \\
\text { and } \\
\text { Hospital }\end{array}$ & $\begin{array}{r}34.8^{\wedge} \\
(25-44)\end{array}$ & 64.4 & 24.5 & - & - & - & - \\
\hline 270 & Zhao, Chen & $\begin{array}{l}2020-07- \\
30\end{array}$ & USA & 641 & Hospital & ${ }^{(N A)}{ }^{60}$ & 40.1 & 21.7 & - & - & - & - \\
\hline 271 & Holman & $\begin{array}{l}2020-08- \\
13\end{array}$ & UK & 10,989 & $\begin{array}{l}\text { Community } \\
\text { and } \\
\text { Hospital }\end{array}$ & NA & 38.8 & 5.5 & 42.6 & - & 49.0 & - \\
\hline 272 & Qu & $\begin{array}{l}2020-07- \\
29\end{array}$ & China & 246 & Hospital & $\begin{array}{r}53.6^{\wedge} \\
(38-68)\end{array}$ & 53.3 & 42.3 & - & - & - & - \\
\hline 273 & Chand & $\begin{array}{l}2020-08- \\
19\end{array}$ & USA & 300 & Hospital & $\begin{array}{r}58.2^{\wedge} \\
(45-70)\end{array}$ & 39.3 & 22.3 & - & - & - & - \\
\hline 274 & Oliveira & $\begin{array}{l}2020-08- \\
31\end{array}$ & USA & 131 & Hospital & $\begin{array}{l}61 \\
(49.5- \\
71.5)\end{array}$ & 64.9 & - & - & 17.6 & 26.7 & - \\
\hline 275 & Hussein, Galal & $\begin{array}{l}2020-09- \\
01\end{array}$ & Egypt & 444 & Community & $\begin{array}{r}33.1^{\wedge} \\
(21-45)\end{array}$ & 56.8 & 13.1 & 9.0 & - & 77.9 & - \\
\hline 276 & Vilar-Garcia & $\begin{array}{l}2020-09- \\
01\end{array}$ & Spain & $7,699,568$ & $\begin{array}{l}\text { Community } \\
\text { and } \\
\text { Hospital }\end{array}$ & $\begin{array}{c}43 \\
(24-59)\end{array}$ & 50.9 & 17.1 & - & - & - & - \\
\hline & & & & & Conmmunitv & & & & & & & \\
\hline
\end{tabular}




\begin{tabular}{|c|c|c|c|c|c|c|c|c|c|c|c|c|}
\hline 277 & Ibarra, Nava & $\begin{array}{l}2020-09- \\
01\end{array}$ & Mexico & 416,546 & $\begin{array}{l}\text { and } \\
\text { Hospital }\end{array}$ & NA & 46.9 & 7.4 & - & - & - & - \\
\hline 278 & Ibrahim & $\begin{array}{l}2020-08- \\
27\end{array}$ & USA & 38 & Hospital & $\begin{array}{c}63^{\wedge} \\
(51-75)\end{array}$ & 47.0 & 10.5 & - & - & - & - \\
\hline 279 & Rubio-Rivas & $\begin{array}{l}2020-09- \\
01\end{array}$ & Spain & 186 & Hospital & $\begin{array}{r}64.3^{\wedge} \\
(51-77)\end{array}$ & 30.6 & 4.3 & 20.4 & - & 75.3 & - \\
\hline 280 & Mamtani & $\begin{array}{l}2020-09- \\
02\end{array}$ & USA & 403 & Hospital & $\begin{array}{c}55^{\wedge} \\
(41-68)\end{array}$ & 32.3 & 9.7 & 12.7 & - & 68.5 & - \\
\hline 281 & Ren & $\begin{array}{l}2020-09- \\
02\end{array}$ & China & 432 & Hospital & NA & 57.9 & 10.0 & - & - & 90.0 & - \\
\hline 282 & Yoo & $\begin{array}{l}2020-08- \\
31\end{array}$ & USA & 4,840 & Hospital & $\begin{array}{l}66.4 \\
(54.9- \\
77.8)\end{array}$ & 43.5 & 4.4 & 21.4 & - & 53.3 & - \\
\hline 283 & Mutambudzi & $\begin{array}{l}2020-09- \\
03\end{array}$ & UK & 120,075 & $\begin{array}{l}\text { Community } \\
\text { and } \\
\text { Hospital }\end{array}$ & NA & 54.2 & 11.7 & 26.4 & - & 61.9 & - \\
\hline 284 & Yan & $\begin{array}{l}2020-09- \\
07\end{array}$ & China & 578 & Hospital & $\begin{array}{c}49.2^{\wedge} \\
(35-63)\end{array}$ & 49.3 & 9.2 & - & - & - & - \\
\hline 285 & $\begin{array}{l}\text { Mancilla- } \\
\text { Galindo }\end{array}$ & $\begin{array}{l}2020-09- \\
08\end{array}$ & Mexico & 183,779 & $\begin{array}{l}\text { Community } \\
\text { and } \\
\text { Hospital }\end{array}$ & $\begin{array}{c}45^{\wedge} \\
(28-61)\end{array}$ & 46.0 & 7.6 & - & - & - & - \\
\hline 286 & Ullah & $\begin{array}{l}2020-09- \\
08\end{array}$ & UK & 212 & $\begin{array}{l}\text { Community } \\
\text { and } \\
\text { Hospital }\end{array}$ & $\begin{array}{l}66.7 \\
(54.2- \\
80.5)\end{array}$ & 44.8 & 11.3 & 48.1 & - & 37.7 & - \\
\hline 256 & Dashti & $\begin{array}{l}2020-09- \\
13\end{array}$ & USA & 12,347 & $\begin{array}{l}\text { Community } \\
\text { and } \\
\text { Hospital }\end{array}$ & $\begin{array}{c}47 \\
(32-62)\end{array}$ & 53.3 & 4.6 & 15.9 & - & 57.1 & - \\
\hline 287 & Nicholson & $\begin{array}{l}2020-09- \\
17\end{array}$ & USA & 1,042 & Hospital & $\begin{array}{c}64 \\
(53-75)\end{array}$ & 43.2 & 8.3 & 22.2 & - & 37.1 & - \\
\hline 288 & Ariza & $\begin{array}{l}2020-09- \\
18\end{array}$ & Colombia & 351 & $\begin{array}{l}\text { Community } \\
\text { and } \\
\text { Hospital }\end{array}$ & $\begin{array}{l}30.5 \\
\text { (NA) }\end{array}$ & 54.0 & 6.8 & - & - & - & 93.2 \\
\hline 37 & Carrat & $\begin{array}{l}2020-09- \\
18\end{array}$ & France & 14,628 & Community & NA & 60.3 & 12.0 & 40.8 & - & 45.6 & - \\
\hline 289 & Zhu & $\begin{array}{l}2020-09- \\
21\end{array}$ & China & 432 & $\begin{array}{l}\text { Community } \\
\text { and } \\
\text { Hospital }\end{array}$ & $\begin{array}{c}49 \\
(35-60)\end{array}$ & 47.9 & 14.4 & - & - & - & - \\
\hline 290 & Sun & $\begin{array}{l}2020-08- \\
16\end{array}$ & USA & 323 & $\begin{array}{l}\text { Community } \\
\text { and } \\
\text { Hospital }\end{array}$ & NA & 57.6 & - & - & 39.3 & - & 60.7 \\
\hline 291 & Kalan & $\begin{array}{l}2020-05- \\
01\end{array}$ & Iran & 193 & Hospital & $\begin{array}{r}52.6^{\wedge} \\
(37-67)\end{array}$ & 36.3 & 7.3 & - & - & 85.0 & - \\
\hline 292 & Burrell & $\begin{array}{l}2020-09- \\
16\end{array}$ & Australia & 204 & Hospital & $\begin{array}{c}63.5 \\
(53-72)\end{array}$ & 31.4 & - & - & 13.2 & - & 82.8 \\
\hline 293 & ISARIC & $\begin{array}{l}2020-09- \\
03\end{array}$ & Multiple & 81,705 & Hospital & (NA) ${ }^{72}$ & 43.0 & - & - & 5.2 & 36.4 & - \\
\hline 294 & Meini & $\begin{array}{l}2020-09- \\
23\end{array}$ & Italy & 461 & Hospital & NA & 51.2 & 10.4 & 25.8 & - & 63.8 & - \\
\hline
\end{tabular}

Note. - Age not provided for total sample; ^ Denotes mean (SD). * This study was rated as 'poor' quality as the manuscript only presents data for current (but not former) smokers despite having obtained complete smoking status, thus resulting in 
$>20 \%$ missing data on smoking status.

\section{Smoking prevalence by country}

Unadjusted smoking prevalence compared with overall estimates for national adult smoking prevalence split by country and study setting is presented in Figure $2 a$ and $2 b$. Lower than expected current smoking prevalence was generally observed. Former smoking prevalence was more similar to expected prevalence when reported. National smoking prevalence estimates used for comparison are presented in Supplementary table 3.

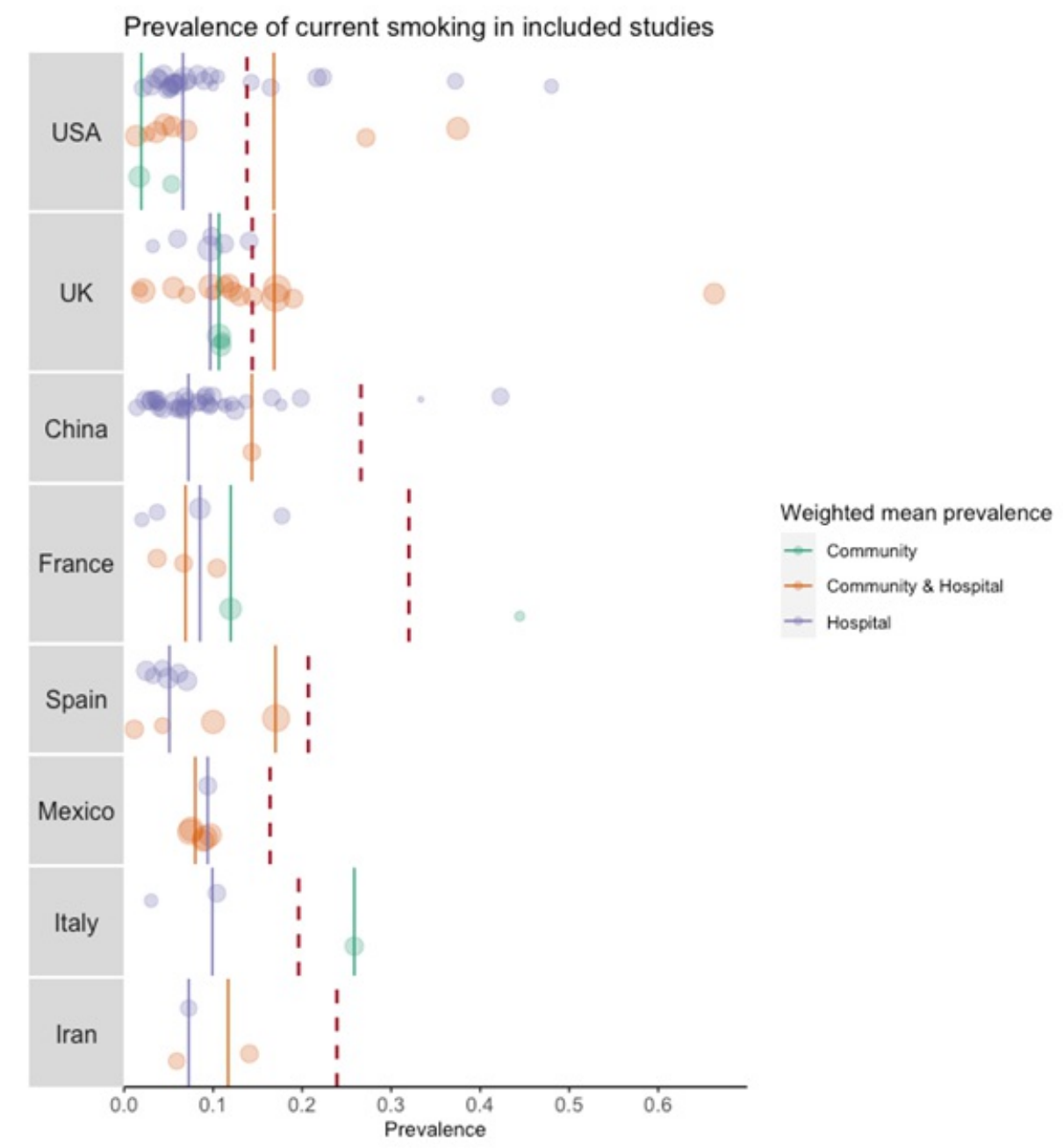

Figure 2a. Weighted mean prevalence of current smoking in included studies, split by country (solid lines). The circles represent individual studies, with colour corresponding to study setting (i.e. community, community and hospital, hospital) and size corresponding to relative study sample size. For comparison, national current smoking prevalence is shown by the dashed red lines. Countries with three or more eligible studies are shown. 


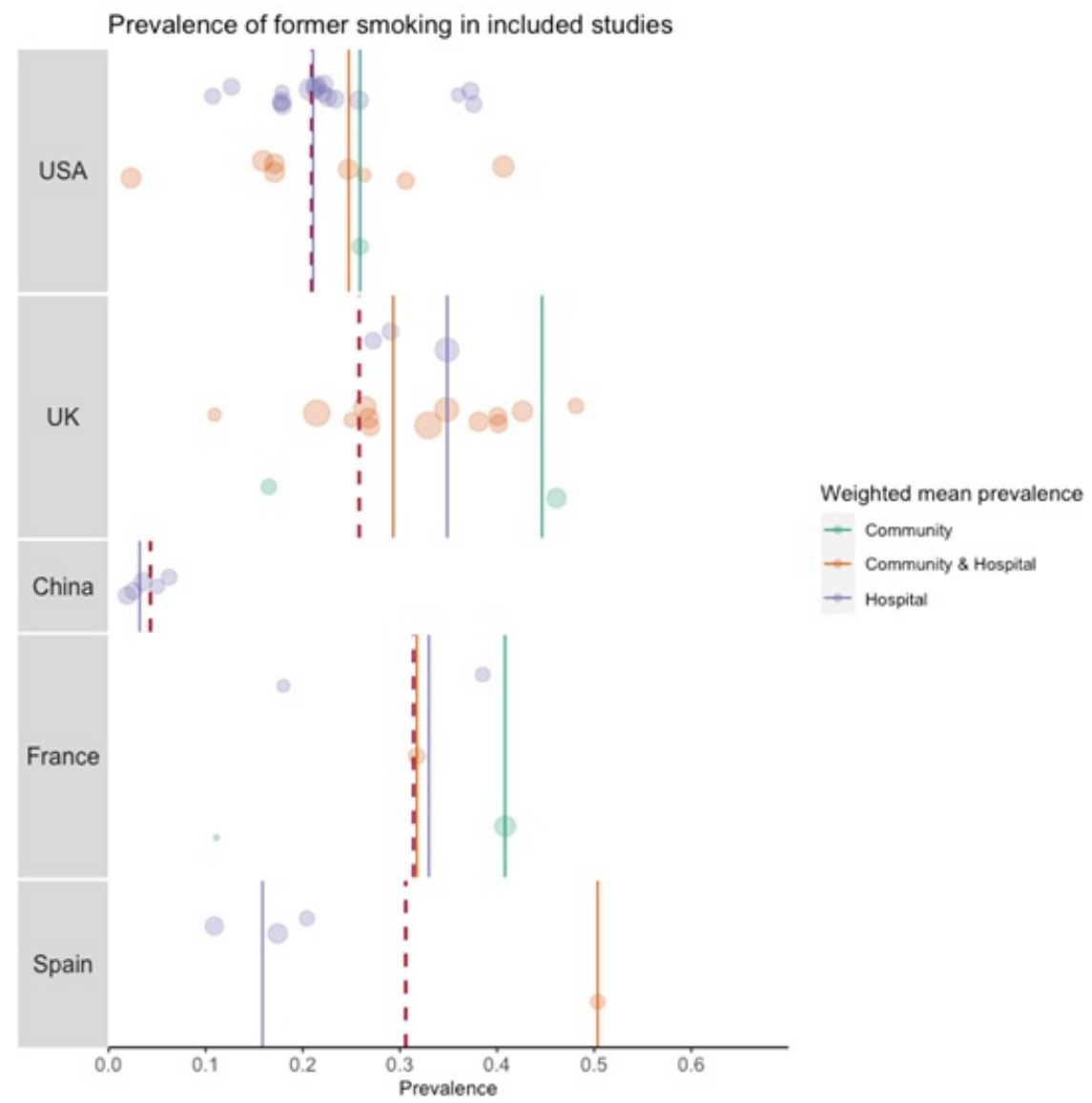

Figure 2b. Prevalence of former smoking in included studies, split by country (solid lines). The circles represent individual studies, with colour corresponding to study setting (i.e. community, community and hospital, hospital) and size corresponding to relative study sample size. For comparison, national former smoking prevalence is shown by dashed red lines. Countries with three or more eligible studies are shown.

\section{SARS-CoV-2 testing by smoking status}

Three studies provided data on access to SARS-CoV-2 diagnostic testing for those meeting local testing criteria by smoking status. In a cohort study of US military veterans aged $54-75^{31}$, current smokers were more likely to receive a test: $42.3 \%(1,603 / 3,789)$ of the sample were current smokers compared with $23.8 \%$ of all veterans aged $50+$ years using any tobacco product between 2010-2015 $5^{32}$. In the UK Biobank cohort ${ }^{33}$, a multivariable analysis showed former $(R R=1.29$, $95 \% \mathrm{Cl}=1.14-1.45, p<.001)$ and current $(\mathrm{RR}=1.44,95 \% \mathrm{Cl}=1.20-1.71, p<.001)$ compared with never smokers to be more likely to receive a test. In an Australian rapid assessment screening clinic for COVID-19 ${ }^{34}, 9.4 \%(397 / 4,226)$ of the self-referred sample (subsequently assessed by a healthcare professional to decide on testing) were current smokers. Of these self-referrals, healthcare professionals decided that current compared with former or never smokers were less likely to require a test $(\mathrm{RR}=0.93,95 \% \mathrm{Cl}=0.86-1.0, p=0.045)$.

\section{SARS-CoV-2 infection by smoking status}


Fifty studies provided data on SARS-CoV-2 infection for people meeting local testing criteria by smoking status (see Table 2). Meta-analyses were performed for two 'good' and 17 'fair' quality studies (see Figure 4 and 5). Current smokers were at reduced risk of testing positive for SARS-CoV-2 compared with never smokers $(R R=0.72,95 \% \mathrm{Crl}=0.57-0.89, \tau=0.40$, $95 \% \mathrm{Cl}=0.25-0.61)$. The probability of current smokers being at reduced risk of infection compared with never smokers (RR $\leq 0.9$ ) was $98 \%$. Former compared with never smokers were at increased risk of testing positive, but data were inconclusive $(\mathrm{RR}=1.02,95 \% \mathrm{Crl}=0.92-1.13, \tau=0.18,95 \% \mathrm{Cl}=0.11-0.27)$ and favoured there being no important association. The probability of former smokers being at increased risk of infection ( $R R \geq 1.1)$ compared with never smokers was $7 \%$. Results were materially unchanged in the two sensitivity analyses (see Supplementary figure S2).

Table 2. SARS-CoV-2 infection by smoking status.

\begin{tabular}{|c|c|c|c|c|c|c|c|c|c|c|c|}
\hline \multirow[b]{2}{*}{ Author } & \multirow[b]{2}{*}{$\begin{array}{l}\text { Total } \\
\text { population } \\
\text { tested }\end{array}$} & \multicolumn{6}{|c|}{ SARS-CoV-2 negative } & \multicolumn{4}{|c|}{ SARS-CoV-2 positive } \\
\hline & & N (\%) & $\begin{array}{l}\text { Current } \\
\text { smoker } \\
(\%)\end{array}$ & $\begin{array}{l}\text { Former } \\
\text { smoker } \\
(\%)\end{array}$ & $\begin{array}{l}\text { Current/former } \\
\text { smoker (\%) }\end{array}$ & $\begin{array}{l}\text { Never } \\
\text { smoker } \\
(\%)\end{array}$ & $\begin{array}{l}\text { Not } \\
\text { stated } \\
(\%)\end{array}$ & N (\%) & $\begin{array}{l}\text { Current } \\
\text { smoker } \\
(\%)\end{array}$ & $\begin{array}{l}\text { Former } \\
\text { smoker } \\
(\%)\end{array}$ & $\begin{array}{l}\text { Current/former } \\
\text { smoker (\%) }\end{array}$ \\
\hline Rentsch & 3528 & $\begin{array}{l}2974 \\
(84.30 \%)\end{array}$ & $\begin{array}{l}1444 \\
(48.55 \%)\end{array}$ & $\begin{array}{l}704 \\
(23.67 \%)\end{array}$ & - & $\begin{array}{l}826 \\
(27.77 \%)\end{array}$ & - & $\begin{array}{l}554 \\
(15.70 \%)\end{array}$ & $\begin{array}{l}159 \\
(28.70 \%)\end{array}$ & $\begin{array}{l}179 \\
(32.31 \%)\end{array}$ & - \\
\hline Fontanet & 661 & $\begin{array}{l}490 \\
(74.13 \%)\end{array}$ & $\begin{array}{l}64 \\
(13.06 \%)\end{array}$ & - & - & $\begin{array}{l}426 \\
(86.94 \%)\end{array}$ & - & $\begin{array}{l}171 \\
(25.87 \%)\end{array}$ & $\begin{array}{l}5 \\
(2.92 \%)\end{array}$ & - & - \\
\hline Cho & 1331 & $\begin{array}{l}793 \\
(59.58 \%)\end{array}$ & $\begin{array}{l}142 \\
(17.91 \%)\end{array}$ & $\begin{array}{l}214 \\
(26.99 \%)\end{array}$ & - & $\begin{array}{l}437 \\
(55.11 \%)\end{array}$ & - & $\begin{array}{l}538 \\
(40.42 \%)\end{array}$ & $\begin{array}{l}111 \\
(20.63 \%)\end{array}$ & $\begin{array}{l}145 \\
(26.95 \%)\end{array}$ & - \\
\hline Shah & 243 & $\begin{array}{l}212 \\
(87.24 \%)\end{array}$ & $\begin{array}{l}52 \\
(24.53 \%)\end{array}$ & $\begin{array}{l}47 \\
(22.17 \%)\end{array}$ & - & $\begin{array}{l}113 \\
(53.30 \%)\end{array}$ & - & $\begin{array}{l}29 \\
(11.93 \%)\end{array}$ & $\begin{array}{l}0 \\
(0.00 \%)\end{array}$ & $\begin{array}{l}9 \\
(31.03 \%)\end{array}$ & - \\
\hline Kolin & 1474 & $\begin{array}{l}805 \\
(54.61 \%)\end{array}$ & $\begin{array}{l}141 \\
(17.52 \%)\end{array}$ & $\begin{array}{l}307 \\
(38.14 \%)\end{array}$ & - & $\begin{array}{l}354 \\
(43.98 \%)\end{array}$ & $3(0.37 \%)$ & $\begin{array}{l}669 \\
(45.39 \%)\end{array}$ & $\begin{array}{l}72 \\
(10.76 \%)\end{array}$ & $\begin{array}{l}285 \\
(42.60 \%)\end{array}$ & - \\
\hline de Lusignan & 3291 & $\begin{array}{l}2740 \\
(83.26 \%)\end{array}$ & $\begin{array}{l}366 \\
(13.36 \%)\end{array}$ & $\begin{array}{l}1450 \\
(52.92 \%)\end{array}$ & - & $\begin{array}{l}924 \\
(33.72 \%)\end{array}$ & - & $\begin{array}{l}551 \\
(16.74 \%)\end{array}$ & $\begin{array}{l}47 \\
(8.53 \%)\end{array}$ & $\begin{array}{l}303 \\
(54.99 \%)\end{array}$ & - \\
\hline Valenti & 789 & $\begin{array}{l}689 \\
(87.33 \%)\end{array}$ & $\begin{array}{l}197 \\
(28.59 \%)\end{array}$ & - & - & - & $\begin{array}{l}492 \\
(71.41 \%)\end{array}$ & $\begin{array}{l}40 \\
(5.07 \%)\end{array}$ & $\begin{array}{l}7 \\
(17.50 \%)\end{array}$ & - & - \\
\hline Parrotta & 76 & $\begin{array}{l}39 \\
(51.32 \%)\end{array}$ & $\begin{array}{l}1 \\
(2.56 \%)\end{array}$ & $\begin{array}{l}10 \\
(25.64 \%)\end{array}$ & - & $\begin{array}{l}27 \\
(69.23 \%)\end{array}$ & $1(2.56 \%)$ & $\begin{array}{l}37 \\
(48.68 \%)\end{array}$ & $\begin{array}{l}1 \\
(2.70 \%)\end{array}$ & $\begin{array}{l}10 \\
(27.03 \%)\end{array}$ & - \\
\hline Berumen & 102875 & $\begin{array}{l}71353 \\
(69.36 \%)\end{array}$ & - & - & 7173 (10.05\%) & $\begin{array}{l}64180 \\
(89.95 \%)\end{array}$ & - & $\begin{array}{l}31522 \\
(30.64 \%)\end{array}$ & - & - & 2748 (8.72\%) \\
\hline Israel & 24906 & $\begin{array}{l}20755 \\
(83.33 \%)\end{array}$ & $\begin{array}{l}3783 \\
(18.23 \%)\end{array}$ & $\begin{array}{l}2671 \\
(12.87 \%)\end{array}$ & - & $\begin{array}{l}14301 \\
(68.90 \%)\end{array}$ & - & $\begin{array}{l}41151 \\
(165.23 \%)\end{array}$ & $\begin{array}{l}406 \\
(0.99 \%)\end{array}$ & $\begin{array}{l}483 \\
(1.17 \%)\end{array}$ & - \\
\hline del Valle & 1108 & $\begin{array}{l}143 \\
(12.91 \%)\end{array}$ & $\begin{array}{l}27 \\
(18.88 \%)\end{array}$ & $\begin{array}{l}53 \\
(37.06 \%)\end{array}$ & - & - & $\begin{array}{l}63 \\
(44.06 \%)\end{array}$ & $\begin{array}{l}965 \\
(87.09 \%)\end{array}$ & $\begin{array}{l}55 \\
(5.70 \%)\end{array}$ & $\begin{array}{l}293 \\
(30.36 \%)\end{array}$ & - \\
\hline Romao & 34 & $\begin{array}{l}20 \\
(58.82 \%)\end{array}$ & - & - & $5(25.00 \%)$ & - & $\begin{array}{l}15 \\
(75.00 \%)\end{array}$ & $\begin{array}{l}14 \\
(41.18 \%)\end{array}$ & - & - & 4 (28.57\%) \\
\hline Ramlall & 11116 & $\begin{array}{l}4723 \\
(42.49 \%)\end{array}$ & - & - & - & - & - & $\begin{array}{l}6393 \\
(57.51 \%)\end{array}$ & - & - & $\begin{array}{l}1643.001 \\
(25.70 \%)\end{array}$ \\
\hline Sharma & 501 & $\begin{array}{l}267 \\
(53.29 \%)\end{array}$ & - & - & $1(0.37 \%)$ & - & $\begin{array}{l}266 \\
(99.63 \%)\end{array}$ & $\begin{array}{l}234 \\
(46.71 \%)\end{array}$ & - & - & 20 (8.55\%) \\
\hline Eugen-Olsen & 407 & $\begin{array}{l}290 \\
(71.25 \%)\end{array}$ & $\begin{array}{l}76 \\
(26.21 \%)\end{array}$ & $\begin{array}{l}104 \\
(35.86 \%)\end{array}$ & - & $\begin{array}{l}102 \\
(35.17 \%)\end{array}$ & - & $\begin{array}{l}117 \\
(28.75 \%)\end{array}$ & $\begin{array}{l}8 \\
(6.84 \%)\end{array}$ & $\begin{array}{l}46 \\
(39.32 \%)\end{array}$ & - \\
\hline $\begin{array}{l}\text { Raisi- } \\
\text { Estabragh }\end{array}$ & 4510 & $\begin{array}{l}3184 \\
(70.60 \%)\end{array}$ & - & - & 1653 (51.92\%) & - & $\begin{array}{l}1531 \\
(48.08 \%)\end{array}$ & $\begin{array}{l}1326 \\
(29.40 \%)\end{array}$ & - & - & 683 (51.51\%) \\
\hline
\end{tabular}




\begin{tabular}{|c|c|c|c|c|c|c|c|c|c|c|c|}
\hline Houlihan & 177 & $\begin{array}{l}97 \\
(54.80 \%)\end{array}$ & $\begin{array}{l}14 \\
(14.43 \%)\end{array}$ & $\begin{array}{l}14 \\
(14.43 \%)\end{array}$ & - & $\begin{array}{l}69 \\
(71.13 \%)\end{array}$ & - & $\begin{array}{l}80 \\
(45.20 \%)\end{array}$ & $\begin{array}{l}7 \\
(8.75 \%)\end{array}$ & $\begin{array}{l}19 \\
(23.75 \%)\end{array}$ & - \\
\hline McQueenie & 428199 & $\begin{array}{l}424355 \\
(99.10 \%)\end{array}$ & - & - & $\begin{array}{l}189299 \\
(44.61 \%)\end{array}$ & $\begin{array}{l}235056 \\
(55.39 \%)\end{array}$ & - & $\begin{array}{l}1311 \\
(0.31 \%)\end{array}$ & - & - & 669 (51.03\%) \\
\hline Woolford & 4474 & $\begin{array}{l}3161 \\
(70.65 \%)\end{array}$ & $\begin{array}{l}441 \\
(13.95 \%)\end{array}$ & $\begin{array}{l}1194 \\
(37.77 \%)\end{array}$ & - & $\begin{array}{l}1526 \\
(48.28 \%)\end{array}$ & - & $\begin{array}{l}1313 \\
(29.35 \%)\end{array}$ & $\begin{array}{l}145 \\
(11.04 \%)\end{array}$ & $\begin{array}{l}525 \\
(39.98 \%)\end{array}$ & - \\
\hline Lan & 104 & $\begin{array}{l}83 \\
(79.81 \%)\end{array}$ & - & - & $24(28.92 \%)$ & - & $\begin{array}{l}59 \\
(71.08 \%)\end{array}$ & $\begin{array}{l}21 \\
(20.19 \%)\end{array}$ & - & - & $1(4.76 \%)$ \\
\hline $\begin{array}{l}\text { Hernandez- } \\
\text { Garduno }\end{array}$ & 32583 & $\begin{array}{l}20279 \\
(62.24 \%)\end{array}$ & - & - & $2399(11.83 \%)$ & $\begin{array}{l}17861 \\
(88.08 \%)\end{array}$ & - & $\begin{array}{l}12304 \\
(37.76 \%)\end{array}$ & - & - & 1191 (9.68\%) \\
\hline Govind & 6215 & $\begin{array}{l}6207 \\
(99.87 \%)\end{array}$ & $\begin{array}{l}4104 \\
(66.12 \%)\end{array}$ & $\begin{array}{l}1669 \\
(26.89 \%)\end{array}$ & - & $\begin{array}{l}342 \\
(5.51 \%)\end{array}$ & - & $\begin{array}{l}102 \\
(1.64 \%)\end{array}$ & $\begin{array}{l}78 \\
(76.47 \%)\end{array}$ & $\begin{array}{l}20 \\
(19.61 \%)\end{array}$ & - \\
\hline Gu & 4699 & $\begin{array}{l}3815 \\
(81.19 \%)\end{array}$ & $\begin{array}{l}360 \\
(9.44 \%)\end{array}$ & $\begin{array}{l}1142 \\
(29.93 \%)\end{array}$ & - & $\begin{array}{l}2313 \\
(60.63 \%)\end{array}$ & - & $\begin{array}{l}884 \\
(18.81 \%)\end{array}$ & $\begin{array}{l}40 \\
(4.52 \%)\end{array}$ & $\begin{array}{l}264 \\
(29.86 \%)\end{array}$ & - \\
\hline Kibler & 702 & $\begin{array}{l}680 \\
(96.87 \%)\end{array}$ & $\begin{array}{l}25 \\
(3.68 \%)\end{array}$ & - & - & - & $\begin{array}{l}655 \\
(96.32 \%)\end{array}$ & $\begin{array}{l}22 \\
(3.13 \%)\end{array}$ & $\begin{array}{l}1 \\
(4.55 \%)\end{array}$ & - & - \\
\hline Petrilli & 10620 & $\begin{array}{l}5341 \\
(50.29 \%)\end{array}$ & $\begin{array}{l}3454 \\
(64.67 \%)\end{array}$ & $\begin{array}{l}816 \\
(15.28 \%)\end{array}$ & - & $\begin{array}{l}541 \\
(10.13 \%)\end{array}$ & $\begin{array}{l}530 \\
(9.92 \%)\end{array}$ & $\begin{array}{l}5279 \\
(49.71 \%)\end{array}$ & $\begin{array}{l}3268 \\
(61.91 \%)\end{array}$ & $\begin{array}{l}902 \\
(17.09 \%)\end{array}$ & - \\
\hline $\begin{array}{l}\text { Bello- } \\
\text { Chavolla }\end{array}$ & 150200 & $\begin{array}{l}98567 \\
(65.62 \%)\end{array}$ & - & - & $9624(9.76 \%)$ & - & $\begin{array}{l}88943 \\
(90.24 \%)\end{array}$ & $\begin{array}{l}51633 \\
(34.38 \%)\end{array}$ & - & - & $4366(8.46 \%)$ \\
\hline Auvinen & 61 & $\begin{array}{l}33 \\
(54.10 \%)\end{array}$ & $\begin{array}{l}10 \\
(30.30 \%)\end{array}$ & $\begin{array}{l}8 \\
(24.24 \%)\end{array}$ & - & $\begin{array}{l}15 \\
(45.45 \%)\end{array}$ & - & $\begin{array}{l}28 \\
(45.90 \%)\end{array}$ & $\begin{array}{l}1 \\
(3.57 \%)\end{array}$ & $\begin{array}{l}9 \\
(32.14 \%)\end{array}$ & - \\
\hline Favara & 70 & $\begin{array}{l}55 \\
(78.57 \%)\end{array}$ & $\begin{array}{l}5 \\
(9.09 \%)\end{array}$ & - & - & - & $\begin{array}{l}50 \\
(90.91 \%)\end{array}$ & $\begin{array}{l}15 \\
(21.43 \%)\end{array}$ & $\begin{array}{l}2 \\
(13.33 \%)\end{array}$ & - & - \\
\hline Antonio-Villa & 34263 & $\begin{array}{l}23338 \\
(68.11 \%)\end{array}$ & $\begin{array}{l}2293 \\
(9.83 \%)\end{array}$ & - & - & - & $\begin{array}{l}21045 \\
(90.17 \%)\end{array}$ & $\begin{array}{l}10925 \\
(31.89 \%)\end{array}$ & $\begin{array}{l}1023 \\
(9.36 \%)\end{array}$ & - & - \\
\hline Merzon & 7807 & $\begin{array}{l}7025 \\
(89.98 \%)\end{array}$ & - & - & $1136(16.17 \%)$ & - & $\begin{array}{l}5889 \\
(83.83 \%)\end{array}$ & $\begin{array}{l}782 \\
(10.02 \%)\end{array}$ & - & - & $127(16.24 \%)$ \\
\hline Trubiano & 2676 & $\begin{array}{l}2827 \\
(105.64 \%)\end{array}$ & - & - & $256(9.06 \%)$ & - & $\begin{array}{l}2586 \\
(91.48 \%)\end{array}$ & $\begin{array}{l}108 \\
(4.04 \%)\end{array}$ & - & - & $3(2.78 \%)$ \\
\hline $\begin{array}{l}\text { Shi, } \\
\text { Resurreccion }\end{array}$ & 1521 & $\begin{array}{l}1265 \\
(83.17 \%)\end{array}$ & - & - & $681(53.83 \%)$ & - & $\begin{array}{l}584 \\
(46.17 \%)\end{array}$ & $\begin{array}{l}256 \\
(16.83 \%)\end{array}$ & - & - & $154(60.16 \%)$ \\
\hline Riley & 120620 & $\begin{array}{l}120461 \\
(99.87 \%)\end{array}$ & $\begin{array}{l}2594 \\
(2.15 \%)\end{array}$ & - & - & $\begin{array}{l}19914 \\
(16.53 \%)\end{array}$ & $\begin{array}{l}97953 \\
(81.32 \%)\end{array}$ & $\begin{array}{l}159 \\
(0.13 \%)\end{array}$ & $\begin{array}{l}3 \\
(1.89 \%)\end{array}$ & - & - \\
\hline Alizadehsani & 319 & $\begin{array}{l}196 \\
(61.44 \%)\end{array}$ & - & - & - & - & $\begin{array}{l}196 \\
(100.00 \%)\end{array}$ & $\begin{array}{l}123 \\
(38.56 \%)\end{array}$ & - & - & $1(0.81 \%)$ \\
\hline Merkely & 10474 & $\begin{array}{l}10336 \\
(98.68 \%)\end{array}$ & $\begin{array}{l}2904 \\
(28.10 \%)\end{array}$ & $\begin{array}{l}2107 \\
(20.39 \%)\end{array}$ & - & $\begin{array}{l}5310 \\
(51.37 \%)\end{array}$ & $\begin{array}{l}15 \\
(0.15 \%)\end{array}$ & $\begin{array}{l}70 \\
(0.67 \%)\end{array}$ & $\begin{array}{l}16 \\
(22.86 \%)\end{array}$ & $\begin{array}{l}15 \\
(21.43 \%)\end{array}$ & - \\
\hline Mcgrail & 209 & $\begin{array}{l}118 \\
(56.46 \%)\end{array}$ & - & - & $31(26.27 \%)$ & - & $\begin{array}{l}87 \\
(73.73 \%)\end{array}$ & $\begin{array}{l}91 \\
(43.54 \%)\end{array}$ & - & - & $8(8.79 \%)$ \\
\hline Izquierdo & 71192 & $\begin{array}{l}\text { NA( } \\
\text { NA\%) }\end{array}$ & - & - & - & - & - & $\begin{array}{l}1006 \\
(1.41 \%)\end{array}$ & $\begin{array}{l}111 \\
(11.03 \%)\end{array}$ & - & - \\
\hline Ward & 99908 & $\begin{array}{l}94416 \\
(94.50 \%)\end{array}$ & $\begin{array}{l}10202 \\
(10.81 \%)\end{array}$ & - & - & - & $\begin{array}{l}84214 \\
(89.19 \%)\end{array}$ & $\begin{array}{l}5492 \\
(5.50 \%)\end{array}$ & $\begin{array}{l}433 \\
(7.88 \%)\end{array}$ & - & - \\
\hline Ebinger & 6062 & $\begin{array}{l}5850 \\
(96.50 \%)\end{array}$ & $\begin{array}{l}99 \\
(1.69 \%)\end{array}$ & - & - & - & $\begin{array}{l}5668 \\
(96.89 \%)\end{array}$ & $\begin{array}{l}212 \\
(3.50 \%)\end{array}$ & $\begin{array}{l}3 \\
(1.42 \%)\end{array}$ & - & - \\
\hline Salerno & 15920 & $\begin{array}{l}14753 \\
(92.67 \%)\end{array}$ & - & - & 5517 (37.40\%) & $\begin{array}{l}8278 \\
(56.11 \%)\end{array}$ & $\begin{array}{l}958 \\
(6.49 \%)\end{array}$ & $\begin{array}{l}1167 \\
(7.33 \%)\end{array}$ & - & - & 339 (29.05\%) \\
\hline Iversen & 28792 & $\begin{array}{l}27629 \\
(95.96 \%)\end{array}$ & $\begin{array}{l}4430 \\
(16.03 \%)\end{array}$ & $\begin{array}{l}1799 \\
(6.51 \%)\end{array}$ & - & $\begin{array}{l}21217 \\
(76.79 \%)\end{array}$ & $\begin{array}{l}246 \\
(0.89 \%)\end{array}$ & $\begin{array}{l}1163 \\
(4.04 \%)\end{array}$ & $\begin{array}{l}177 \\
(15.22 \%)\end{array}$ & $\begin{array}{l}78 \\
(6.71 \%)\end{array}$ & - \\
\hline Hippislev- & & NAI & & & & & & 19486 & 1354 & 5715 & \\
\hline
\end{tabular}




\begin{tabular}{|c|c|c|c|c|c|c|c|c|c|c|c|}
\hline Cox & 8275949 & NA\%) & - & - & - & - & - & $(0.24 \%)$ & $(6.95 \%)$ & $(29.33 \%)$ & - \\
\hline Fillmore & 22914 & $\begin{array}{l}21120 \\
(92.17 \%)\end{array}$ & $\begin{array}{l}8137 \\
(38.53 \%)\end{array}$ & $\begin{array}{l}8416 \\
(39.85 \%)\end{array}$ & - & $\begin{array}{l}3227 \\
(15.28 \%)\end{array}$ & $\begin{array}{l}1340 \\
(6.34 \%)\end{array}$ & $\begin{array}{l}1794 \\
(7.83 \%)\end{array}$ & $\begin{array}{l}452 \\
(25.20 \%)\end{array}$ & $\begin{array}{l}899 \\
(50.11 \%)\end{array}$ & - \\
\hline Alkurt & 119 & $\begin{array}{l}\text { NA ( } \\
\text { NA\%) }\end{array}$ & - & - & - & - & - & $\begin{array}{l}119 \\
(100.00 \%)\end{array}$ & $\begin{array}{l}14 \\
(11.76 \%)\end{array}$ & - & - \\
\hline Ariza & 351 & $\begin{array}{l}322 \\
(91.74 \%)\end{array}$ & $\begin{array}{l}21 \\
(6.52 \%)\end{array}$ & - & - & - & $\begin{array}{l}301 \\
(93.48 \%)\end{array}$ & $\begin{array}{l}29 \\
(8.26 \%)\end{array}$ & $\begin{array}{l}3 \\
(10.34 \%)\end{array}$ & - & - \\
\hline Carrat & 14393 & $\begin{array}{l}13426 \\
(93.28 \%)\end{array}$ & $\begin{array}{l}1652 \\
(12.30 \%)\end{array}$ & $\begin{array}{l}5620 \\
(41.86 \%)\end{array}$ & - & $\begin{array}{l}6154 \\
(45.84 \%)\end{array}$ & - & $\begin{array}{l}967 \\
(6.72 \%)\end{array}$ & $\begin{array}{l}98 \\
(10.13 \%)\end{array}$ & $\begin{array}{l}353 \\
(36.50 \%)\end{array}$ & - \\
\hline Meini & 461 & $\begin{array}{l}243 \\
(52.71 \%)\end{array}$ & $\begin{array}{l}39 \\
(16.05 \%)\end{array}$ & $\begin{array}{l}66 \\
(27.16 \%)\end{array}$ & - & $\begin{array}{l}138 \\
(56.79 \%)\end{array}$ & - & $\begin{array}{l}218 \\
(47.29 \%)\end{array}$ & $\begin{array}{l}9 \\
(4.13 \%)\end{array}$ & $\begin{array}{l}53 \\
(24.31 \%)\end{array}$ & - \\
\hline & & & & & & & & & & & 1 \\
\hline
\end{tabular}

Note. Niedzwiedz et al. reported on SARS-CoV-2 infection by smoking status in multivariable analyses but did not present raw data.

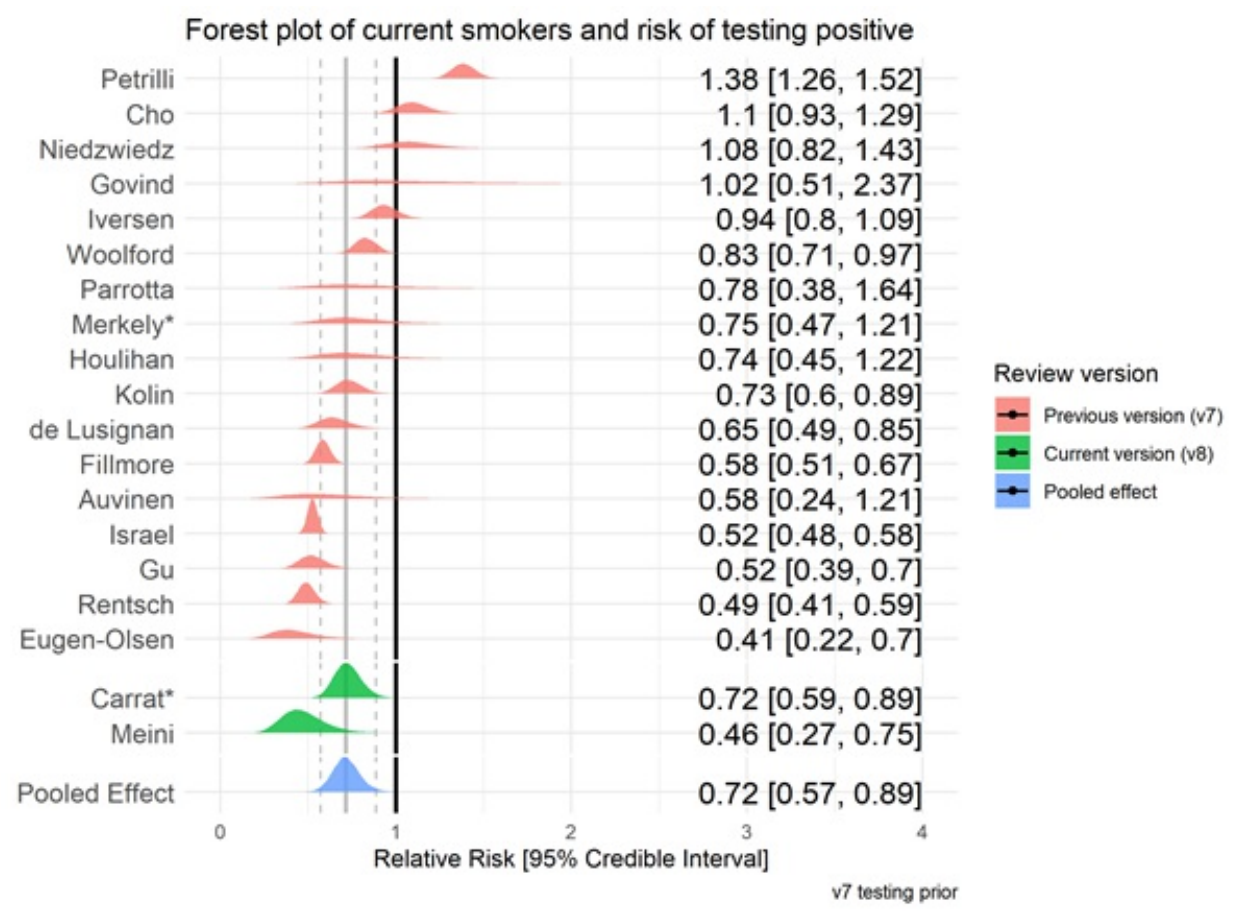

Figure 3. Forest plot for risk of testing positive for SARS-CoV-2 in current vs. never smokers. * Indicates 'good' quality studies. Prior from previous version (v7) RR $=0.74$. 


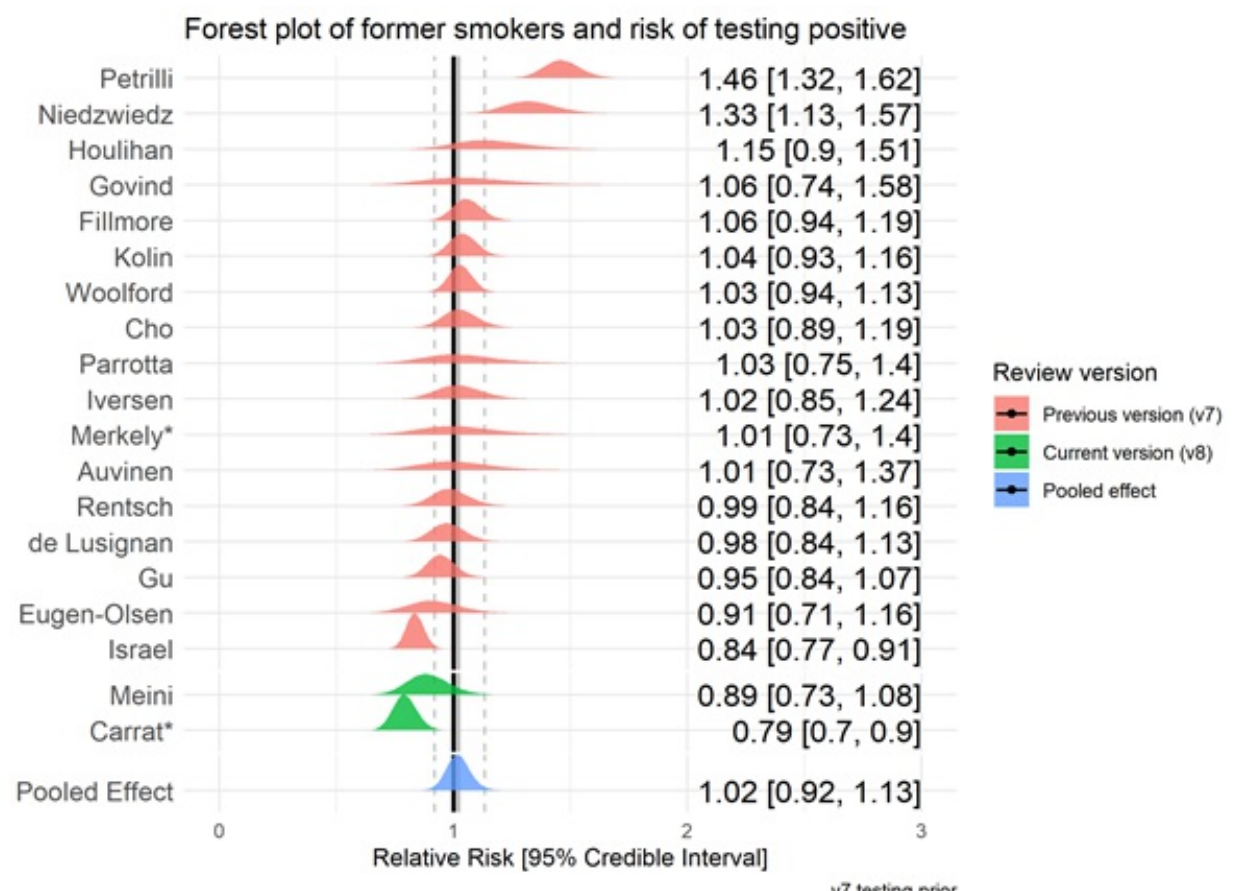

Figure 4. Forest plot for risk of testing positive for SARS-CoV-2 in former vs. never smokers. * Indicates 'good' quality studies. Prior from previous version (v7) RR =1.05.

\section{Hospitalisation for COVID-19 by smoking status}

Thirty-one studies examined hospitalisation for COVID-19 disease, stratified by smoking status (see Table 3). Metaanalyses were performed for eight 'fair' quality studies (see Figure 6 and 7 ). Current ( $R R=1.06, C r l=0.82-1.35, \tau=0.27$, $95 \% \mathrm{Cl}=0.08-0.55)$ and former $(\mathrm{RR}=1.19, \mathrm{Crl}=1.03-1.43, \tau=0.17,95 \% \mathrm{Cl}=0.06-0.37)$ compared with never smokers were at increased risk of hospitalisation with COVID-19. However, data for current smokers were inconclusive and favoured there being no important association. The probability of current and former smokers being at increased risk of hospitalisation ( $R R \geq 1.1$ ) compared with never smokers was $35 \%$ and $89 \%$, respectively. Results were materially unchanged in two sensitivity analyses (see Supplementary figure S3).

Table 3. Hospitalisation with COVID-19 by smoking status.

\begin{tabular}{|c|c|c|c|c|c|c|c|c|c|c|c|c|}
\hline \multirow[b]{2}{*}{ Author } & \multirow[b]{2}{*}{$\begin{array}{l}\text { Population } \\
\text { with } \\
\text { outcome }\end{array}$} & \multicolumn{7}{|c|}{ Community } & \multicolumn{4}{|c|}{ Hospitalised } \\
\hline & & N (\%) & $\begin{array}{l}\text { Current } \\
\text { smoker } \\
(\%)\end{array}$ & $\begin{array}{l}\text { Former } \\
\text { smoker } \\
(\%)\end{array}$ & $\begin{array}{l}\text { Current/former } \\
\text { smoker (\%) }\end{array}$ & $\begin{array}{l}\text { Never } \\
\text { smoker } \\
(\%)\end{array}$ & $\begin{array}{l}\text { Never/unknown } \\
\text { smoker (\%) }\end{array}$ & $\begin{array}{l}\text { Not } \\
\text { stated } \\
(\%)\end{array}$ & N (\%) & $\begin{array}{l}\text { Current } \\
\text { smoker } \\
(\%)\end{array}$ & $\begin{array}{l}\text { Former } \\
\text { smoker } \\
(\%)\end{array}$ & $\begin{array}{l}\text { Cur } \\
\text { sme }\end{array}$ \\
\hline Rentsch & 554 & $\begin{array}{l}269 \\
(48 \%)\end{array}$ & $\begin{array}{l}69 \\
(25.65 \%)\end{array}$ & $\begin{array}{l}90 \\
(33.46 \%)\end{array}$ & - & $\begin{array}{l}110 \\
(40.89 \%)\end{array}$ & - & - & $\begin{array}{l}285 \\
(51 \%)\end{array}$ & $\begin{array}{l}90 \\
(31.58 \%)\end{array}$ & $\begin{array}{l}89 \\
(31.23 \%)\end{array}$ & - \\
\hline $\begin{array}{l}\text { Chow (US } \\
\text { CDC) }\end{array}$ & 6637 & $\begin{array}{l}5143 \\
(77 \%)\end{array}$ & $\begin{array}{l}61 \\
(1.19 \%)\end{array}$ & $\begin{array}{l}80 \\
(1.56 \%)\end{array}$ & - & - & - & $\begin{array}{l}5002 \\
(97.26 \%)\end{array}$ & $\begin{array}{l}1494 \\
(22 \%)\end{array}$ & $\begin{array}{l}27 \\
(1.81 \%)\end{array}$ & $\begin{array}{l}78 \\
(5.22 \%)\end{array}$ & - \\
\hline Argenziano & 1000 & $\begin{array}{l}151 \\
(15 \%)\end{array}$ & $\begin{array}{l}14 \\
(9.27 \%)\end{array}$ & $\begin{array}{l}18 \\
(11.92 \%)\end{array}$ & - & $\begin{array}{l}119 \\
(78.81 \%)\end{array}$ & - & - & $\begin{array}{l}849 \\
(84 \%)\end{array}$ & $\begin{array}{l}35 \\
(4.12 \%)\end{array}$ & $\begin{array}{l}161 \\
(18.96 \%)\end{array}$ & - \\
\hline Lubetzky & 54 & $\begin{array}{l}15 \\
(27 \%)\end{array}$ & - & - & $4(26.67 \%)$ & - & - & $\begin{array}{l}11 \\
(73.33 \%)\end{array}$ & $\begin{array}{l}39 \\
(72 \%)\end{array}$ & - & - & $8(2$ \\
\hline
\end{tabular}




\begin{tabular}{|c|c|c|c|c|c|c|c|c|c|c|c|c|}
\hline Carillo-Vega & 9946 & $\begin{array}{l}3922 \\
(39 \%)\end{array}$ & $\begin{array}{l}408 \\
(10.40 \%)\end{array}$ & - & - & - & - & $\begin{array}{l}3514 \\
(89.60 \%)\end{array}$ & $\begin{array}{l}6024 \\
(60 \%)\end{array}$ & $\begin{array}{l}486 \\
(8.07 \%)\end{array}$ & - & - \\
\hline Yanover & 4353 & $\begin{array}{l}4180 \\
(96 \%)\end{array}$ & $\begin{array}{l}484 \\
(11.58 \%)\end{array}$ & $\begin{array}{l}118 \\
(2.82 \%)\end{array}$ & - & $\begin{array}{l}3578 \\
(85.60 \%)\end{array}$ & - & - & $\begin{array}{l}173 \\
(3 \%)\end{array}$ & $\begin{array}{l}30 \\
(17.34 \%)\end{array}$ & $\begin{array}{l}11 \\
(6.36 \%)\end{array}$ & - \\
\hline Hamer & 387109 & $\begin{array}{l}386349 \\
(99 \%)\end{array}$ & $\begin{array}{l}37333 \\
(9.66 \%)\end{array}$ & $\begin{array}{l}134542 \\
(34.82 \%)\end{array}$ & - & $\begin{array}{l}214474 \\
(55.51 \%)\end{array}$ & - & - & $\begin{array}{l}760 \\
(0 \%)\end{array}$ & $\begin{array}{l}93 \\
(12.24 \%)\end{array}$ & $\begin{array}{l}313 \\
(41.18 \%)\end{array}$ & - \\
\hline Heili-Frades & 4712 & $\begin{array}{l}1973 \\
(41 \%)\end{array}$ & $\begin{array}{l}121 \\
(6.13 \%)\end{array}$ & $\begin{array}{l}222 \\
(11.25 \%)\end{array}$ & - & - & $1630(82.62 \%)$ & $\begin{array}{l}1630 \\
(82.62 \%)\end{array}$ & $\begin{array}{l}2739 \\
(58 \%)\end{array}$ & $\begin{array}{l}112 \\
(4.09 \%)\end{array}$ & $\begin{array}{l}598 \\
(21.83 \%)\end{array}$ & - \\
\hline Freites & 123 & $\begin{array}{l}69 \\
(56 \%)\end{array}$ & $\begin{array}{l}1 \\
(1.45 \%)\end{array}$ & - & - & - & - & $\begin{array}{l}68 \\
(98.55 \%)\end{array}$ & $\begin{array}{l}54 \\
(43 \%)\end{array}$ & $\begin{array}{l}3 \\
(5.56 \%)\end{array}$ & - & - \\
\hline Berumen & 102875 & $\begin{array}{l}18832 \\
(18 \%)\end{array}$ & - & - & $1546(8.21 \%)$ & - & $17286(91.79 \%)$ & - & $\begin{array}{l}12690 \\
(12 \%)\end{array}$ & - & - & 120 \\
\hline Gianfrancesco & 600 & $\begin{array}{l}323 \\
(53 \%)\end{array}$ & - & - & $61(18.89 \%)$ & - & - & $\begin{array}{l}262 \\
(81.11 \%)\end{array}$ & $\begin{array}{l}277 \\
(46 \%)\end{array}$ & - & - & 68( \\
\hline Chaudhry & 40 & $\begin{array}{l}19 \\
(47 \%)\end{array}$ & - & - & $0(0.00 \%)$ & - & - & $\begin{array}{l}19 \\
(100.00 \%)\end{array}$ & $\begin{array}{l}21 \\
(52 \%)\end{array}$ & - & - & $6(2$ \\
\hline Giannouchos & 89756 & $\begin{array}{l}58485 \\
(65 \%)\end{array}$ & $\begin{array}{l}4679 \\
(8.00 \%)\end{array}$ & - & - & - & $53806(92.00 \%)$ & - & $\begin{array}{l}31271 \\
(34 \%)\end{array}$ & $\begin{array}{l}2721 \\
(8.70 \%)\end{array}$ & - & - \\
\hline $\begin{array}{l}\text { Wang, } \\
\text { Oekelen }\end{array}$ & 57 & $\begin{array}{l}22 \\
(38 \%)\end{array}$ & - & - & $6(27.27 \%)$ & - & - & $\begin{array}{l}16 \\
(72.73 \%)\end{array}$ & $\begin{array}{l}36 \\
(63 \%)\end{array}$ & - & - & 15( \\
\hline Miyara & 470 & $\begin{array}{l}132 \\
(28 \%)\end{array}$ & $\begin{array}{l}14 \\
(10.61 \%)\end{array}$ & $\begin{array}{l}41 \\
(31.06 \%)\end{array}$ & - & $\begin{array}{l}77 \\
(58.33 \%)\end{array}$ & - & - & $\begin{array}{l}338 \\
(71 \%)\end{array}$ & $\begin{array}{l}18 \\
(5.33 \%)\end{array}$ & $\begin{array}{l}111 \\
(32.84 \%)\end{array}$ & - \\
\hline Suleyman & 463 & $\begin{array}{l}108 \\
(23 \%)\end{array}$ & - & - & $23(21.30 \%)$ & - & - & $\begin{array}{l}85 \\
(78.70 \%)\end{array}$ & $\begin{array}{l}355 \\
(76 \%)\end{array}$ & - & - & 137 \\
\hline Garassino & 196 & $\begin{array}{l}48 \\
(24 \%)\end{array}$ & $\begin{array}{l}10 \\
(20.83 \%)\end{array}$ & $\begin{array}{l}27 \\
(56.25 \%)\end{array}$ & - & $\begin{array}{l}11 \\
(22.92 \%)\end{array}$ & - & - & $\begin{array}{l}152 \\
(77 \%)\end{array}$ & $\begin{array}{l}38 \\
(25.00 \%)\end{array}$ & $\begin{array}{l}84 \\
(55.26 \%)\end{array}$ & - \\
\hline Siso-Almirall & 260 & $\begin{array}{l}119 \\
(45 \%)\end{array}$ & - & - & $31(26.05 \%)$ & - & - & $\begin{array}{l}88 \\
(73.95 \%)\end{array}$ & $\begin{array}{l}141 \\
(54 \%)\end{array}$ & - & - & 50( \\
\hline $\mathrm{Gu}$ & 884 & $\begin{array}{l}511 \\
(57 \%)\end{array}$ & $\begin{array}{l}30 \\
(5.87 \%)\end{array}$ & $\begin{array}{l}126 \\
(24.66 \%)\end{array}$ & - & $\begin{array}{l}355 \\
(69.47 \%)\end{array}$ & - & - & $\begin{array}{l}373 \\
(42 \%)\end{array}$ & $\begin{array}{l}10 \\
(2.68 \%)\end{array}$ & $\begin{array}{l}138 \\
(37.00 \%)\end{array}$ & - \\
\hline Killerby & 531 & $\begin{array}{l}311 \\
(58 \%)\end{array}$ & - & - & 37 (11.90\%) & $\begin{array}{l}222 \\
(71.38 \%)\end{array}$ & - & $\begin{array}{l}52 \\
(16.72 \%)\end{array}$ & $\begin{array}{l}220 \\
(41 \%)\end{array}$ & - & - & 54( \\
\hline Petrilli & 5279 & $\begin{array}{l}2538 \\
(48 \%)\end{array}$ & $\begin{array}{l}147 \\
(5.79 \%)\end{array}$ & $\begin{array}{l}337 \\
(13.28 \%)\end{array}$ & - & $\begin{array}{l}1678 \\
(66.12 \%)\end{array}$ & - & $\begin{array}{l}376 \\
(14.81 \%)\end{array}$ & $\begin{array}{l}2741 \\
(51 \%)\end{array}$ & $\begin{array}{l}141 \\
(5.14 \%)\end{array}$ & $\begin{array}{l}565 \\
(20.61 \%)\end{array}$ & - \\
\hline Nguyen & 689 & $\begin{array}{l}333 \\
(48 \%)\end{array}$ & - & - & 57 (17.12\%) & - & - & $\begin{array}{l}276 \\
(82.88 \%)\end{array}$ & $\begin{array}{l}356 \\
(51 \%)\end{array}$ & - & - & 114 \\
\hline Mendy & 689 & $\begin{array}{l}473 \\
(68 \%)\end{array}$ & - & - & 84 (17.76\%) & - & - & $\begin{array}{l}389 \\
(82.24 \%)\end{array}$ & $\begin{array}{l}216 \\
(31 \%)\end{array}$ & - & - & 86( \\
\hline Soares & 10713 & $\begin{array}{l}9561 \\
(89 \%)\end{array}$ & $\begin{array}{l}132 \\
(1.38 \%)\end{array}$ & - & - & - & 9429 (98.62\%) & - & $\begin{array}{l}1152 \\
(10 \%)\end{array}$ & $\begin{array}{l}77 \\
(6.68 \%)\end{array}$ & - & - \\
\hline Zobairy & 203 & $\begin{array}{l}65 \\
(32 \%)\end{array}$ & $\begin{array}{l}1 \\
(1.54 \%)\end{array}$ & - & - & - & $64(98.46 \%)$ & - & $\begin{array}{l}138 \\
(67 \%)\end{array}$ & $\begin{array}{l}11 \\
(7.97 \%)\end{array}$ & - & - \\
\hline Izquierdo & 1006 & $\begin{array}{l}743 \\
(73 \%)\end{array}$ & $\begin{array}{l}52 \\
(7.00 \%)\end{array}$ & - & - & - & 691 (93.00\%) & - & $\begin{array}{l}263 \\
(26 \%)\end{array}$ & $\begin{array}{l}16 \\
(6.08 \%)\end{array}$ & - & - \\
\hline Rizzo & 76819 & $\begin{array}{l}60039 \\
(78 \%)\end{array}$ & $\begin{array}{l}3931 \\
(6.55 \%)\end{array}$ & $\begin{array}{l}11379 \\
(18.95 \%)\end{array}$ & - & $\begin{array}{l}30042 \\
(50.04 \%)\end{array}$ & - & $\begin{array}{l}14687 \\
(24.46 \%)\end{array}$ & $\begin{array}{l}16780 \\
(21 \%)\end{array}$ & $\begin{array}{l}1254 \\
(7.47 \%)\end{array}$ & $\begin{array}{l}4585 \\
(27.32 \%)\end{array}$ & - \\
\hline Dashti & 4140 & $\begin{array}{l}2759 \\
(66 \%)\end{array}$ & - & - & 600 (21.75\%) & $\begin{array}{l}1541 \\
(55.85 \%)\end{array}$ & - & $\begin{array}{l}618 \\
(22.40 \%)\end{array}$ & $\begin{array}{l}1381 \\
(33 \%)\end{array}$ & - & - & 577 \\
\hline Pan & 12084 & $\begin{array}{l}8548 \\
(70 \%)\end{array}$ & - & - & $1263(14.78 \%)$ & - & - & $\begin{array}{l}7285 \\
(85.22 \%)\end{array}$ & $\begin{array}{l}3536 \\
(29 \%)\end{array}$ & - & - & 874 \\
\hline
\end{tabular}




\begin{tabular}{|c|c|c|c|c|c|c|c|c|c|}
\hline Vilar-Garcia & 328892 & $\begin{array}{l}291254 \\
(88 \%)\end{array}$ & $\begin{array}{l}64792 \\
(22.25 \%)\end{array}$ & - & - & $\begin{array}{l}226462 \\
(77.75 \%)\end{array}$ & $\begin{array}{l}37638 \\
(11 \%)\end{array}$ & $\begin{array}{l}9526 \\
(25.31 \%)\end{array}$ & - \\
\hline Ibarra-Nava & 416546 & $\begin{array}{l}302693 \\
(72 \%)\end{array}$ & $\begin{array}{l}26773 \\
(8.84 \%)\end{array}$ & - & - & $\begin{array}{l}275920 \\
(91.16 \%)\end{array}$ & $\begin{array}{l}113853 \\
(27 \%)\end{array}$ & $\begin{array}{l}8875 \\
(7.80 \%)\end{array}$ & - \\
\hline Dashti & 12347 & $\begin{array}{l}8946 \\
(72 \%)\end{array}$ & $\begin{array}{l}353 \\
(3.95 \%)\end{array}$ & $\begin{array}{l}1099 \\
(12.28 \%)\end{array}$ & $\begin{array}{l}5133 \\
(57.38 \%)\end{array}$ & $\begin{array}{l}2361 \\
(26.39 \%)\end{array}$ & $\begin{array}{l}3401 \\
(27 \%)\end{array}$ & $\begin{array}{l}210 \\
(6.17 \%)\end{array}$ & $\begin{array}{l}860 \\
(25.29 \%)\end{array}$ \\
\hline
\end{tabular}

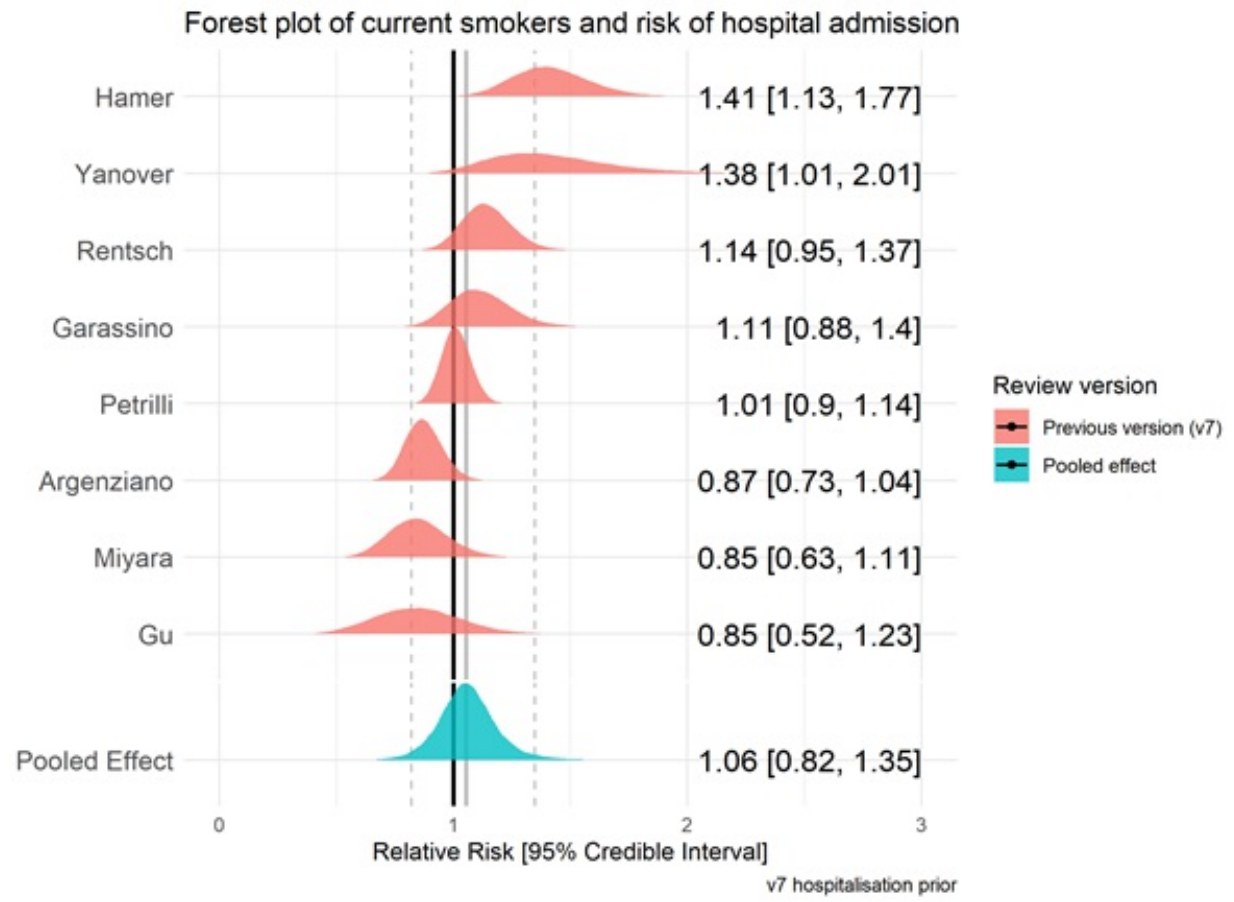

Figure 5. Forest plot for risk of hospitalisation in current vs. never smokers. Prior from previous version (v7) RR $=1.06$

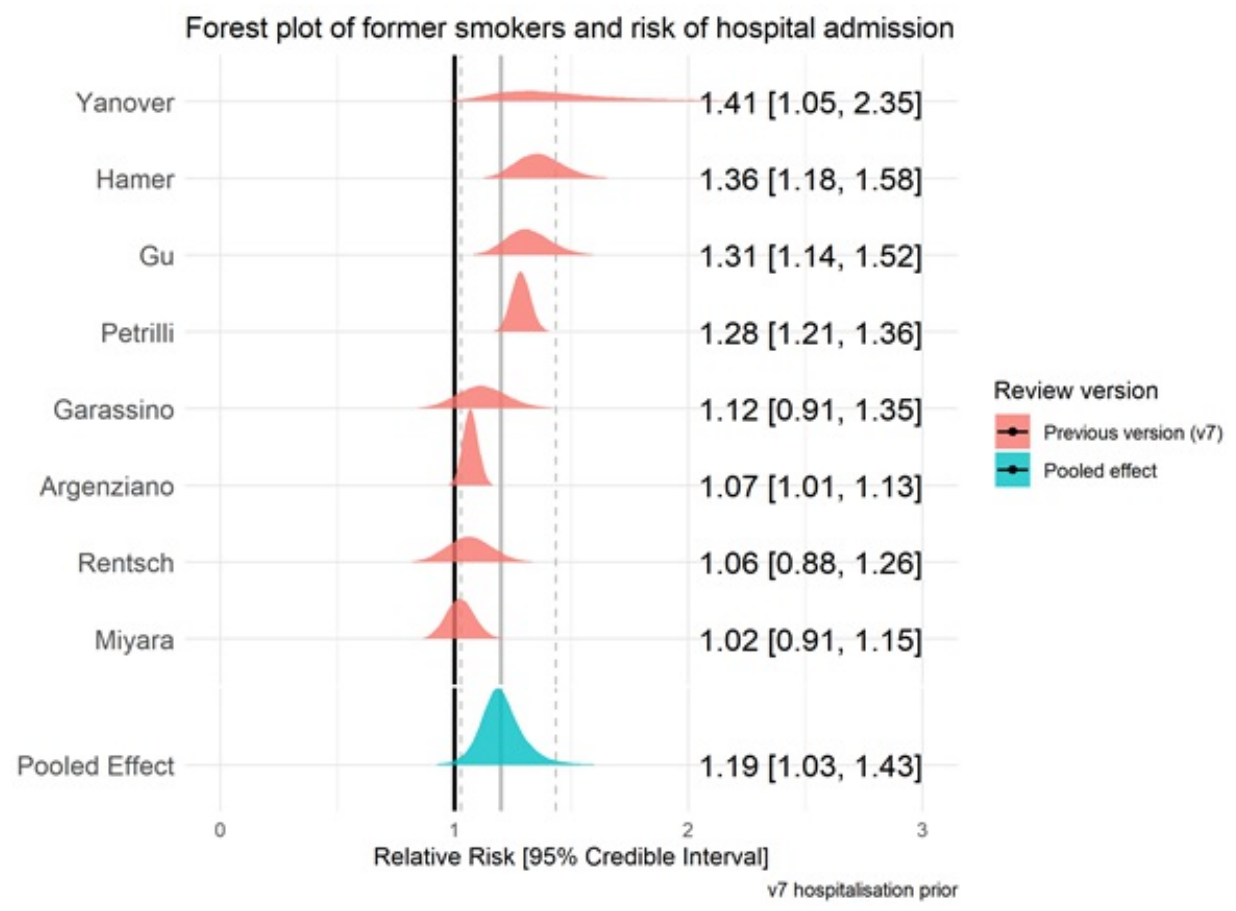


Figure 6. Forest plot for risk of hospitalisation in former vs. never smokers. Prior from previous version (v7) RR =1.2

\section{Disease severity by smoking status}

Sixty-three studies reported disease severity in hospitalised patients stratified by smoking status (see Table 4). Severe (as opposed to non-severe) disease was broadly defined as requiring intensive treatment unit (ITU) admission, requiring oxygen as a hospital inpatient or in-hospital death. Meta-analyses were performed for eight 'fair' quality studies (see Figure 8 and 9). Current $(\mathrm{RR}=1.26, \mathrm{Crl}=0.85-1.96, \tau=0.34,95 \% \mathrm{Cl}=0.01-0.86)$ and former $(\mathrm{RR}=1.52, \mathrm{Crl}=1.12-2.05, \tau=$ $0.29,95 \% \mathrm{Cl}=0.47-0.66)$ compared with never smokers were at increased risk of greater disease severity; data for current smokers were inconclusive but favoured there being a small but important association. The probability of current and former smokers having increased risk of greater disease severity $(R R \geq 1.1)$ compared with never smokers was $79 \%$ and $98 \%$, respectively. Results were materially unchanged in two sensitivity analyses (see Supplementary figure S4).

Table 4. Disease severity by smoking status.

\begin{tabular}{|c|c|c|c|c|c|c|c|c|c|c|c|c|}
\hline \multirow[b]{2}{*}{ Author } & \multirow[b]{2}{*}{$\begin{array}{l}\text { Population } \\
\text { with } \\
\text { severity }\end{array}$} & \multicolumn{7}{|c|}{ Non severe disease } & \multicolumn{4}{|c|}{ Severe disease } \\
\hline & & $\mathrm{N}(\%)$ & $\begin{array}{l}\text { Current } \\
\text { smoker } \\
(\%)\end{array}$ & $\begin{array}{l}\text { Former } \\
\text { smoker } \\
(\%)\end{array}$ & $\begin{array}{l}\text { Current/former } \\
\text { smoker (\%) }\end{array}$ & $\begin{array}{l}\text { Never } \\
\text { smoker } \\
(\%)\end{array}$ & $\begin{array}{l}\text { Never/unknown } \\
\text { smoker (\%) }\end{array}$ & $\begin{array}{l}\text { Not } \\
\text { stated } \\
(\%)\end{array}$ & $\mathrm{N}(\%)$ & $\begin{array}{l}\text { Current } \\
\text { smoker } \\
(\%)\end{array}$ & $\begin{array}{l}\text { Former } \\
\text { smoker } \\
(\%)\end{array}$ & $\begin{array}{l}\text { C } \\
\text { si }\end{array}$ \\
\hline Guan, Ni & 1085 & $\begin{array}{l}913 \\
(84 \%)\end{array}$ & $\begin{array}{l}108 \\
(11.83 \%)\end{array}$ & $\begin{array}{l}12 \\
(1.31 \%)\end{array}$ & - & $\begin{array}{l}793 \\
(86.86 \%)\end{array}$ & - & - & $\begin{array}{l}172 \\
(15 \%)\end{array}$ & $\begin{array}{l}29 \\
(16.86 \%)\end{array}$ & $\begin{array}{l}9 \\
(5.23 \%)\end{array}$ & - \\
\hline Zhang, Dong & 9 & $\begin{array}{l}3 \\
(33 \%)\end{array}$ & $0(0.00 \%)$ & $\begin{array}{l}3 \\
(100.00 \%)\end{array}$ & - & $\begin{array}{l}0 \\
(0.00 \%)\end{array}$ & - & - & $\begin{array}{l}6 \\
(66 \%)\end{array}$ & $\begin{array}{l}2 \\
\text { (33.33\%) }\end{array}$ & $\begin{array}{l}4 \\
(66.67 \%)\end{array}$ & - \\
\hline Wan & 9 & $\begin{array}{l}8 \\
(88 \%)\end{array}$ & $\begin{array}{l}8 \\
(100.00 \%)\end{array}$ & $0(0.00 \%)$ & - & $\begin{array}{l}0 \\
(0.00 \%)\end{array}$ & - & - & $\begin{array}{l}1 \\
(11 \%)\end{array}$ & $\begin{array}{l}1 \\
(100.00 \%)\end{array}$ & $\begin{array}{l}0 \\
(0.00 \%)\end{array}$ & - \\
\hline $\begin{array}{l}\text { Huang, } \\
\text { Wang }\end{array}$ & 3 & $\begin{array}{l}3 \\
(100 \%)\end{array}$ & $\begin{array}{l}3 \\
(100.00 \%)\end{array}$ & $0(0.00 \%)$ & - & $\begin{array}{l}0 \\
(0.00 \%)\end{array}$ & - & - & $0(0 \%)$ & $0(-\%)$ & $0(-\%)$ & - \\
\hline Rentsch & 285 & $\begin{array}{l}168 \\
(58 \%)\end{array}$ & $\begin{array}{l}47 \\
(27.98 \%)\end{array}$ & $\begin{array}{l}53 \\
(31.55 \%)\end{array}$ & - & $\begin{array}{l}68 \\
(40.48 \%)\end{array}$ & - & - & $\begin{array}{l}117 \\
(41 \%)\end{array}$ & $\begin{array}{l}43 \\
(36.75 \%)\end{array}$ & $\begin{array}{l}36 \\
(30.77 \%)\end{array}$ & - \\
\hline $\mathrm{Hu}$ & 323 & $\begin{array}{l}151 \\
(46 \%)\end{array}$ & - & - & $12(7.95 \%)$ & - & 139 (92.05\%) & - & $\begin{array}{l}172 \\
(53 \%)\end{array}$ & - & - & 26 \\
\hline Wang, Pan & 125 & $\begin{array}{l}100 \\
(80 \%)\end{array}$ & - & - & $9(9.00 \%)$ & - & $91(91.00 \%)$ & - & $\begin{array}{l}25 \\
(20 \%)\end{array}$ & - & - & 7 \\
\hline Kim & 27 & $\begin{array}{l}21 \\
(77 \%)\end{array}$ & $\begin{array}{l}3 \\
(14.29 \%)\end{array}$ & - & - & - & $18(85.71 \%)$ & - & $\begin{array}{l}6 \\
(22 \%)\end{array}$ & $\begin{array}{l}2 \\
(33.33 \%)\end{array}$ & $\begin{array}{l}0 \\
(0.00 \%)\end{array}$ & - \\
\hline Shi, Yu & 474 & $\begin{array}{l}425 \\
(89 \%)\end{array}$ & - & - & 34 (8.00\%) & - & 391 (92.00\%) & - & $\begin{array}{l}49 \\
(10 \%)\end{array}$ & - & - & 6 \\
\hline Liao, Feng & 148 & $\begin{array}{l}92 \\
(62 \%)\end{array}$ & - & - & $5(5.43 \%)$ & - & - & $\begin{array}{l}87 \\
(94.57 \%)\end{array}$ & $\begin{array}{l}56 \\
(37 \%)\end{array}$ & $3(5.36 \%)$ & - & - \\
\hline Shi, Ren & 134 & $\begin{array}{l}88 \\
(65 \%)\end{array}$ & - & - & $8(9.09 \%)$ & - & - & $\begin{array}{l}80 \\
(90.91 \%)\end{array}$ & $\begin{array}{l}46 \\
(34 \%)\end{array}$ & - & - & 6 \\
\hline Hadjadj & 50 & $\begin{array}{l}15 \\
(30 \%)\end{array}$ & $1(6.67 \%)$ & $\begin{array}{l}2 \\
(13.33 \%)\end{array}$ & - & $\begin{array}{l}12 \\
(80.00 \%)\end{array}$ & - & - & $\begin{array}{l}35 \\
(70 \%)\end{array}$ & $0(0.00 \%)$ & $\begin{array}{l}7 \\
(20.00 \%)\end{array}$ & - \\
\hline \multirow[t]{2}{*}{ Zheng, Xiong } & 73 & $\begin{array}{l}43 \\
(58 \%)\end{array}$ & - & - & $6(13.95 \%)$ & $\begin{array}{l}37 \\
(86.05 \%)\end{array}$ & - & - & $\begin{array}{l}30 \\
(41 \%)\end{array}$ & - & - & 2 \\
\hline & & 26 & & & & & & 20 & 20 & & & \\
\hline
\end{tabular}




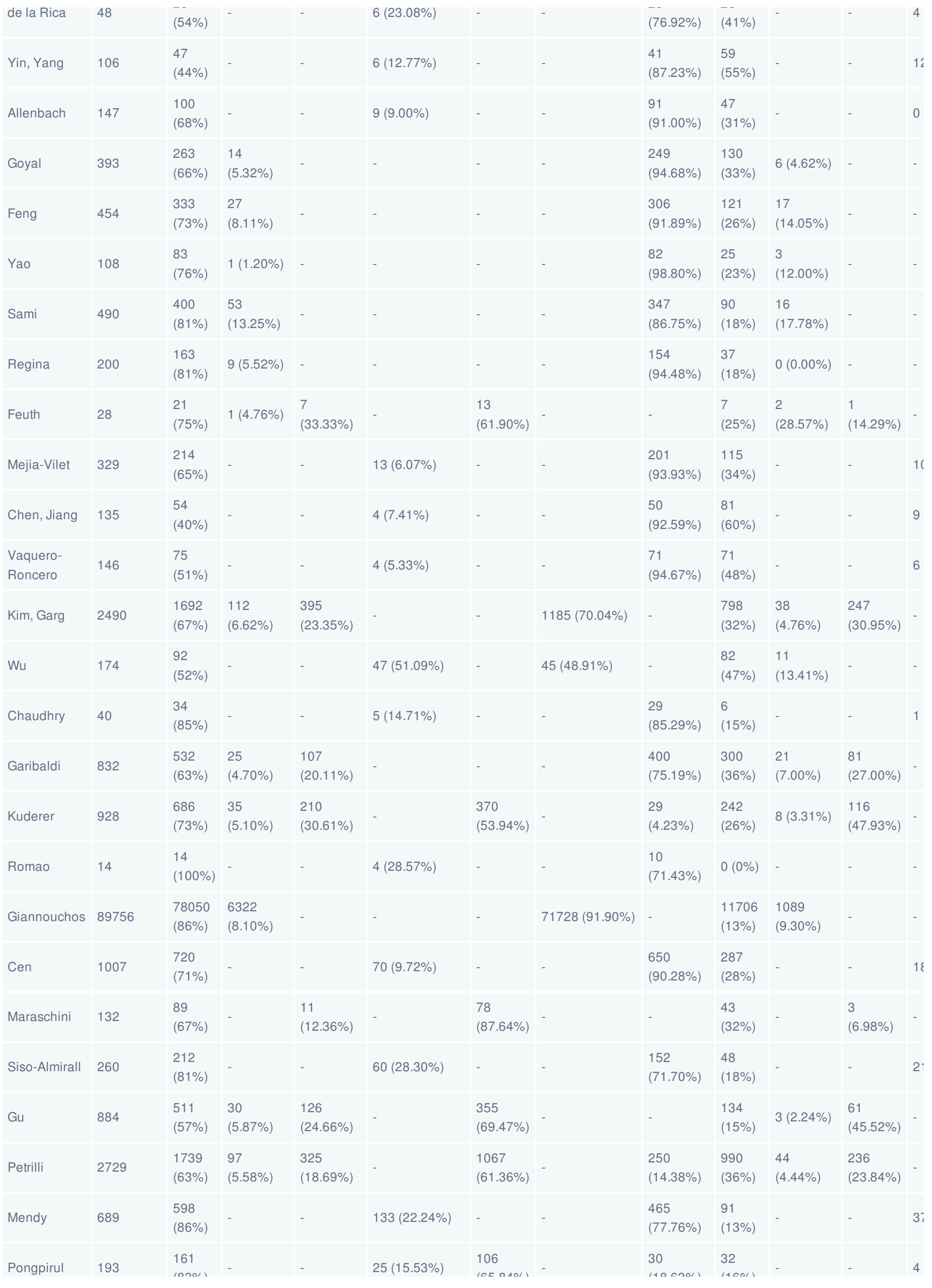




\begin{tabular}{|c|c|c|c|c|c|c|c|c|c|c|c|c|}
\hline & & $(00 \%)$ & & & & $(00.04 \%)$ & & $(10.00 \%)$ & $(10 \%)$ & & & \\
\hline Jin, Gu & 6 & $\begin{array}{l}2 \\
(33 \%)\end{array}$ & - & - & $0(0.00 \%)$ & - & - & $\begin{array}{l}4 \\
(200.00 \%)\end{array}$ & $\begin{array}{l}4 \\
(66 \%)\end{array}$ & - & - & 2 \\
\hline Senkal & 611 & $\begin{array}{l}446 \\
(73 \%)\end{array}$ & $\begin{array}{l}48 \\
(10.76 \%)\end{array}$ & - & - & - & - & $\begin{array}{l}398 \\
(89.24 \%)\end{array}$ & $\begin{array}{l}165 \\
(27 \%)\end{array}$ & $\begin{array}{l}21 \\
(12.73 \%)\end{array}$ & - & - \\
\hline Patel & 129 & $\begin{array}{l}89 \\
(68 \%)\end{array}$ & $\begin{array}{l}26 \\
(29.21 \%)\end{array}$ & - & - & - & 58 (65.17\%) & 5 (5.62\%) & $\begin{array}{l}40 \\
(31 \%)\end{array}$ & $\begin{array}{l}22 \\
(55.00 \%)\end{array}$ & - & - \\
\hline Maucourant & 27 & $\begin{array}{l}10 \\
(37 \%)\end{array}$ & $\begin{array}{l}1 \\
(10.00 \%)\end{array}$ & $\begin{array}{l}2 \\
(20.00 \%)\end{array}$ & - & $\begin{array}{l}2 \\
(20.00 \%)\end{array}$ & - & $\begin{array}{l}5 \\
(50.00 \%)\end{array}$ & $\begin{array}{l}17 \\
(62 \%)\end{array}$ & $\begin{array}{l}2 \\
(11.76 \%)\end{array}$ & $\begin{array}{l}5 \\
(29.41 \%)\end{array}$ & - \\
\hline Xie & 619 & $\begin{array}{l}469 \\
(75 \%)\end{array}$ & - & - & 32 (6.82\%) & - & - & $\begin{array}{l}437 \\
(93.18 \%)\end{array}$ & $\begin{array}{l}150 \\
(24 \%)\end{array}$ & - & - & 1 s \\
\hline Fox & 55 & $\begin{array}{l}30 \\
(54 \%)\end{array}$ & $1(3.33 \%)$ & $\begin{array}{l}4 \\
(13.33 \%)\end{array}$ & - & $\begin{array}{l}17 \\
(56.67 \%)\end{array}$ & - & $\begin{array}{l}8 \\
(26.67 \%)\end{array}$ & $\begin{array}{l}25 \\
(45 \%)\end{array}$ & $0(0.00 \%)$ & $\begin{array}{l}2 \\
(8.00 \%)\end{array}$ & - \\
\hline Zhang, Cao & 240 & $\begin{array}{l}162 \\
(67 \%)\end{array}$ & $2(1.23 \%)$ & $6(3.70 \%)$ & - & - & - & $\begin{array}{l}154 \\
(95.06 \%)\end{array}$ & $\begin{array}{l}78 \\
(32 \%)\end{array}$ & $4(5.13 \%)$ & $\begin{array}{l}4 \\
(5.13 \%)\end{array}$ & - \\
\hline Kurashima & 53 & $\begin{array}{l}10 \\
(18 \%)\end{array}$ & - & - & 3 (30.00\%) & - & - & $\begin{array}{l}7 \\
(70.00 \%)\end{array}$ & $\begin{array}{l}43 \\
(81 \%)\end{array}$ & - & - & 24 \\
\hline Zhan & 75 & $\begin{array}{l}\text { NA } \\
\text { (NA\%) }\end{array}$ & - & - & - & - & - & - & $\begin{array}{l}75 \\
(100 \%)\end{array}$ & - & - & 9 \\
\hline Omrani & 858 & $\begin{array}{l}806 \\
(93 \%)\end{array}$ & - & - & $121(15.01 \%)$ & - & - & $\begin{array}{l}685 \\
(84.99 \%)\end{array}$ & $\begin{array}{l}52 \\
(6 \%)\end{array}$ & - & - & 9 \\
\hline Marcos & 918 & $\begin{array}{l}555 \\
(60 \%)\end{array}$ & $\begin{array}{l}38 \\
(6.85 \%)\end{array}$ & - & 69 (12.43\%) & - & - & $\begin{array}{l}448 \\
(80.72 \%)\end{array}$ & $\begin{array}{l}363 \\
(39 \%)\end{array}$ & $\begin{array}{l}18 \\
(4.96 \%)\end{array}$ & - & $7^{\circ}$ \\
\hline $\begin{array}{l}\text { Hoertel, } \\
\text { Sanchez- } \\
\text { Rico }\end{array}$ & 7345 & $\begin{array}{l}6014 \\
(81 \%)\end{array}$ & $\begin{array}{l}433 \\
(7.20 \%)\end{array}$ & - & - & - & - & $\begin{array}{l}5581 \\
(92.80 \%)\end{array}$ & $\begin{array}{l}1331 \\
(18 \%)\end{array}$ & $\begin{array}{l}190 \\
(14.27 \%)\end{array}$ & - & - \\
\hline Qi & 267 & $\begin{array}{l}217 \\
(81 \%)\end{array}$ & $\begin{array}{l}22 \\
(10.14 \%)\end{array}$ & - & - & - & 195 (89.86\%) & - & $\begin{array}{l}50 \\
(18 \%)\end{array}$ & $\begin{array}{l}31 \\
(62.00 \%)\end{array}$ & - & - \\
\hline Monteiro & 112 & $\begin{array}{l}84 \\
(75 \%)\end{array}$ & $3(3.57 \%)$ & $\begin{array}{l}14 \\
(16.67 \%)\end{array}$ & - & $\begin{array}{l}63 \\
(75.00 \%)\end{array}$ & - & $4(4.76 \%)$ & $\begin{array}{l}28 \\
(25 \%)\end{array}$ & $\begin{array}{l}4 \\
(14.29 \%)\end{array}$ & $\begin{array}{l}6 \\
(21.43 \%)\end{array}$ & - \\
\hline Dashti & 1381 & $\begin{array}{l}619 \\
(44 \%)\end{array}$ & - & - & $239(38.61 \%)$ & $\begin{array}{l}292 \\
(47.17 \%)\end{array}$ & - & $\begin{array}{l}88 \\
(14.22 \%)\end{array}$ & $\begin{array}{l}762 \\
(55 \%)\end{array}$ & - & - & $3 i$ \\
\hline Morshed & 103 & $\begin{array}{l}87 \\
(84 \%)\end{array}$ & $\begin{array}{l}28 \\
(32.18 \%)\end{array}$ & - & - & - & 59 (67.82\%) & - & $\begin{array}{l}16 \\
(15 \%)\end{array}$ & $\begin{array}{l}4 \\
(25.00 \%)\end{array}$ & - & - \\
\hline Zhou, Sun & 144 & $\begin{array}{l}108 \\
(75 \%)\end{array}$ & $\begin{array}{l}11 \\
(10.19 \%)\end{array}$ & - & - & - & - & $\begin{array}{l}97 \\
(89.81 \%)\end{array}$ & $\begin{array}{l}36 \\
(25 \%)\end{array}$ & 2 (5.56\%) & - & - \\
\hline $\begin{array}{l}\text { Hippisley- } \\
\text { Cox }\end{array}$ & - & NA & - & - & - & - & - & - & 1286 & $\begin{array}{l}56 \\
(4.35 \%)\end{array}$ & $\begin{array}{l}427 \\
(33.20 \%)\end{array}$ & - \\
\hline Zhao, Chen & 641 & $\begin{array}{l}398 \\
(62 \%)\end{array}$ & $\begin{array}{l}87 \\
(21.86 \%)\end{array}$ & - & - & - & - & $\begin{array}{l}311 \\
(78.14 \%)\end{array}$ & $\begin{array}{l}195 \\
(30 \%)\end{array}$ & $\begin{array}{l}52 \\
(26.67 \%)\end{array}$ & - & - \\
\hline Qu & 246 & $\begin{array}{l}226 \\
(91 \%)\end{array}$ & $\begin{array}{l}90 \\
(39.82 \%)\end{array}$ & - & - & - & - & $\begin{array}{l}136 \\
(60.18 \%)\end{array}$ & $\begin{array}{l}20 \\
(8 \%)\end{array}$ & $\begin{array}{l}14 \\
(70.00 \%)\end{array}$ & - & - \\
\hline Ren & 432 & $\begin{array}{l}314 \\
(72 \%)\end{array}$ & $\begin{array}{l}26 \\
(8.28 \%)\end{array}$ & - & - & $\begin{array}{l}288 \\
(91.72 \%)\end{array}$ & - & - & $\begin{array}{l}118 \\
(27 \%)\end{array}$ & $\begin{array}{l}17 \\
(14.41 \%)\end{array}$ & - & - \\
\hline Yan & 578 & $\begin{array}{l}450 \\
(77 \%)\end{array}$ & $\begin{array}{l}31 \\
(6.89 \%)\end{array}$ & - & - & - & - & $\begin{array}{l}419 \\
(93.11 \%)\end{array}$ & $\begin{array}{l}128 \\
(22 \%)\end{array}$ & $\begin{array}{l}20 \\
(15.62 \%)\end{array}$ & - & - \\
\hline Nicholson & 1042 & $\begin{array}{l}550 \\
(52 \%)\end{array}$ & $\begin{array}{l}37 \\
(6.73 \%)\end{array}$ & $\begin{array}{l}106 \\
(19.27 \%)\end{array}$ & - & $\begin{array}{l}211 \\
(38.36 \%)\end{array}$ & - & $\begin{array}{l}196 \\
(35.64 \%)\end{array}$ & $\begin{array}{l}401 \\
(38 \%)\end{array}$ & $\begin{array}{l}41 \\
(10.22 \%)\end{array}$ & $\begin{array}{l}92 \\
(22.94 \%)\end{array}$ & - \\
\hline Zhu & 432 & $\begin{array}{l}285 \\
(65 \%)\end{array}$ & $\begin{array}{l}46 \\
(16.14 \%)\end{array}$ & - & - & - & - & $\begin{array}{l}239 \\
(83.86 \%)\end{array}$ & $\begin{array}{l}147 \\
(34 \%)\end{array}$ & $\begin{array}{l}16 \\
(10.88 \%)\end{array}$ & - & - \\
\hline
\end{tabular}


$(63 \%)$

85

Burrell

204

(41\%)
$(83.61 \%)$

$7(8.24 \%)$
$(9.02 \%)$

$(36 \%)$

$3(3.53 \%)$

119

(58\%)

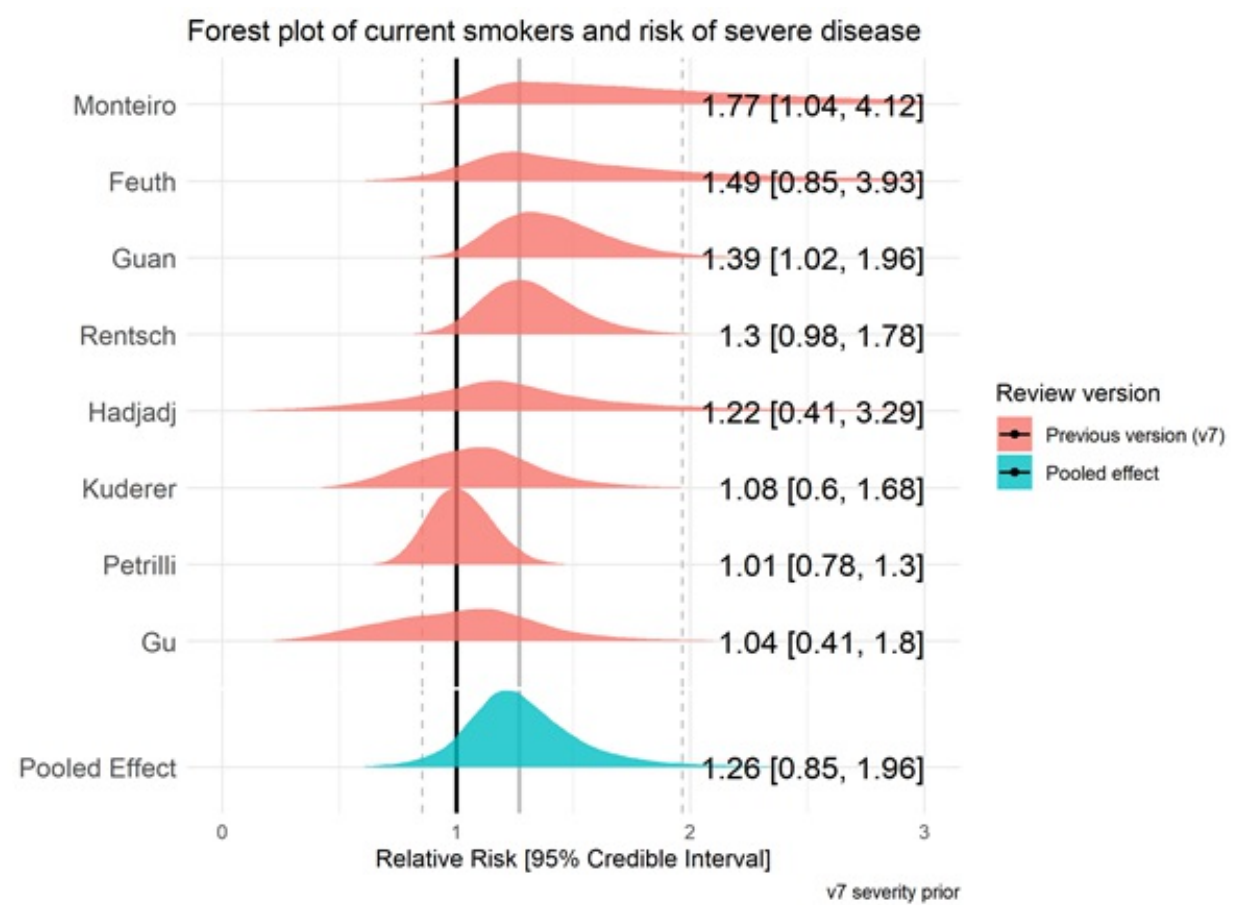

Figure 7. Forest plot for the risk of severe disease in current vs. never smokers. Prior from previous version (v7) RR $=1.25$

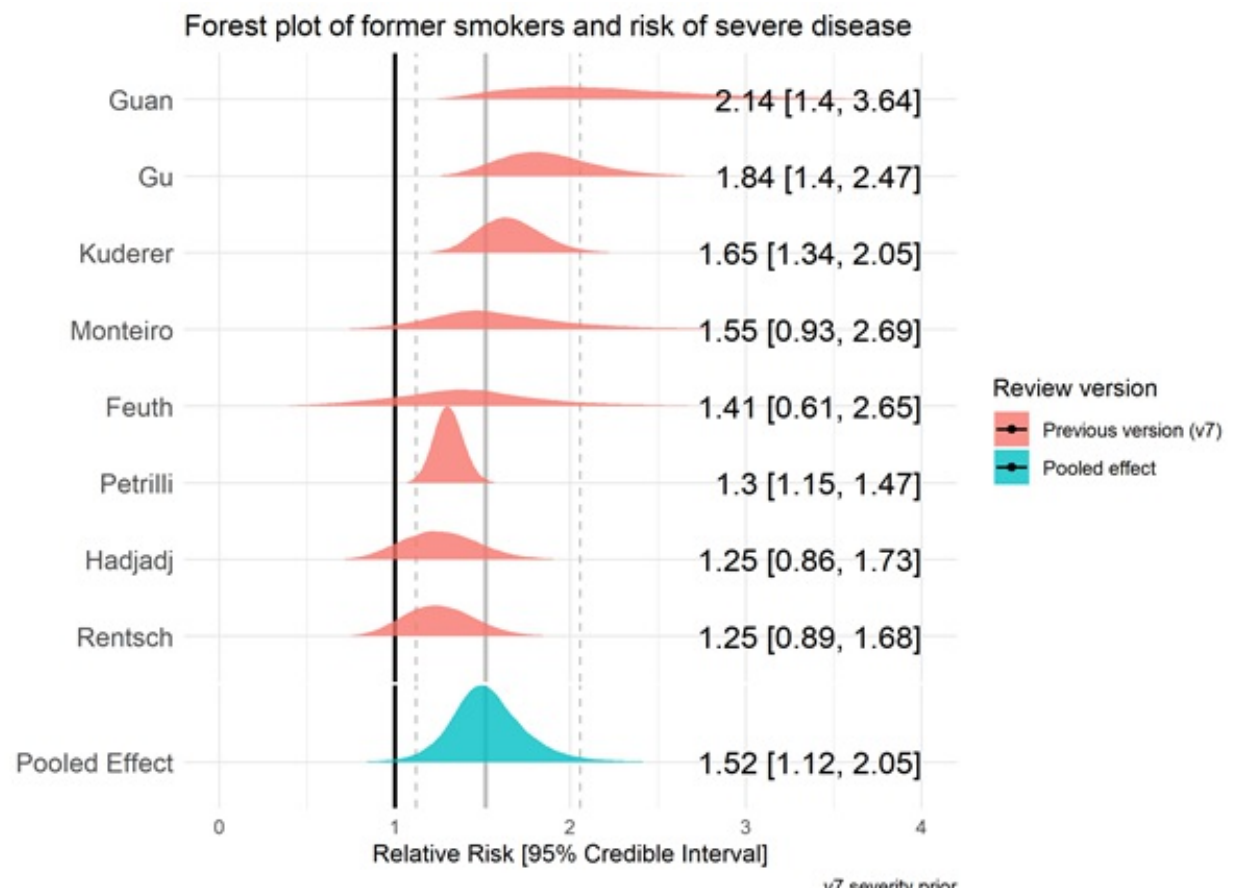

Figure 8. Forest plot for the risk of severe disease in former vs. never smokers. Prior from previous version (v7) $R R=1.52$ 


\section{Mortality by smoking status}

Fifty-eight studies reported mortality from COVID-19 by smoking status (see Table 5), with eleven 'fair' quality studies included in meta-analyses (see Figure 10 and 11). Current $(R R=1.10,95 \% \mathrm{Crl}=0.69-1.67, \tau=0.50,95 \% \mathrm{Cl}=0.17-0.96)$ and former $(\mathrm{RR}=1.35,95 \% \mathrm{Crl}=1.09-1.73, \tau=0.26,95 \% \mathrm{Cl}=0.04-0.52)$ compared with never smokers were at increased risk of in-hospital mortality from COVID-19. Data for current smokers were inconclusive and favoured there being no important association. The probability of current and former smokers being at greater risk of in-hospital mortality ( $R R \geq 1.1$ ) compared with never smokers was $51 \%$ and $97 \%$, respectively. Results were materially unchanged in two sensitivity analyses (see Supplementary figure S5).

Table 5. Mortality by smoking status.

\begin{tabular}{|c|c|c|c|c|c|c|c|c|c|c|c|c|}
\hline \multirow[b]{2}{*}{ Author } & \multirow[b]{2}{*}{$\begin{array}{l}\text { Population } \\
\text { with } \\
\text { mortality }\end{array}$} & \multicolumn{7}{|c|}{ Recovered } & \multicolumn{4}{|l|}{ Died } \\
\hline & & $\mathrm{N}(\%)$ & $\begin{array}{l}\text { Current } \\
\text { smoker } \\
(\%)\end{array}$ & $\begin{array}{l}\text { Former } \\
\text { smoker } \\
\text { (\%) }\end{array}$ & $\begin{array}{l}\text { Current/former } \\
\text { smoker (\%) }\end{array}$ & $\begin{array}{l}\text { Never } \\
\text { smoker } \\
(\%)\end{array}$ & $\begin{array}{l}\text { Never/unknown } \\
\text { smoker (\%) }\end{array}$ & $\begin{array}{l}\text { Not } \\
\text { stated } \\
(\%)\end{array}$ & $\mathrm{N}(\%)$ & $\begin{array}{l}\text { Current } \\
\text { smoker } \\
(\%)\end{array}$ & $\begin{array}{l}\text { Former } \\
\text { smoker } \\
(\%)\end{array}$ & $\begin{array}{l}\text { Curre } \\
\text { smok }\end{array}$ \\
\hline Chen & 274 & $\begin{array}{l}161 \\
(58 \%)\end{array}$ & $\begin{array}{l}5 \\
(3.11 \%)\end{array}$ & $\begin{array}{l}5 \\
(3.11 \%)\end{array}$ & - & - & - & $\begin{array}{l}151 \\
(93.79 \%)\end{array}$ & $\begin{array}{l}113 \\
(41 \%)\end{array}$ & $\begin{array}{l}7 \\
(6.19 \%)\end{array}$ & $\begin{array}{l}2 \\
(1.77 \%)\end{array}$ & - \\
\hline Zhou, Yu & 191 & $\begin{array}{l}137 \\
(71 \%)\end{array}$ & $\begin{array}{l}6 \\
(4.38 \%)\end{array}$ & - & - & - & - & $\begin{array}{l}131 \\
(95.62 \%)\end{array}$ & $\begin{array}{l}54 \\
(28 \%)\end{array}$ & $\begin{array}{l}5 \\
(9.26 \%)\end{array}$ & - & - \\
\hline Yang, Yu & 52 & $\begin{array}{l}20 \\
(38 \%)\end{array}$ & $\begin{array}{l}2 \\
(10.00 \%)\end{array}$ & - & - & - & 18 (90.00\%) & - & $\begin{array}{l}32 \\
(61 \%)\end{array}$ & - & - & - \\
\hline Borobia & 2226 & $\begin{array}{l}1766 \\
(79 \%)\end{array}$ & $\begin{array}{l}113 \\
(6.40 \%)\end{array}$ & - & - & - & - & $\begin{array}{l}1653 \\
(93.60 \%)\end{array}$ & $\begin{array}{l}460 \\
(20 \%)\end{array}$ & $\begin{array}{l}44 \\
(9.57 \%)\end{array}$ & - & - \\
\hline Giacomelli & 233 & $\begin{array}{l}185 \\
(79 \%)\end{array}$ & - & - & $53(28.65 \%)$ & $\begin{array}{l}132 \\
(71.35 \%)\end{array}$ & - & - & $\begin{array}{l}48 \\
(20 \%)\end{array}$ & - & - & $17(35$ \\
\hline Yao & 108 & $\begin{array}{l}96 \\
(88 \%)\end{array}$ & $\begin{array}{l}1 \\
(1.04 \%)\end{array}$ & - & - & - & - & $\begin{array}{l}95 \\
(98.96 \%)\end{array}$ & $\begin{array}{l}12 \\
(11 \%)\end{array}$ & $\begin{array}{l}3 \\
(25.00 \%)\end{array}$ & - & - \\
\hline Carillo-Vega & 9946 & $\begin{array}{l}8983 \\
(90 \%)\end{array}$ & $\begin{array}{l}795 \\
(8.85 \%)\end{array}$ & - & - & - & - & $\begin{array}{l}8188 \\
(91.15 \%)\end{array}$ & $\begin{array}{l}963 \\
(9 \%)\end{array}$ & $\begin{array}{l}99 \\
(10.28 \%)\end{array}$ & - & - \\
\hline Heng & 51 & $\begin{array}{l}39 \\
(76 \%)\end{array}$ & $\begin{array}{l}6 \\
(15.38 \%)\end{array}$ & - & - & - & - & $\begin{array}{l}33 \\
(84.62 \%)\end{array}$ & $\begin{array}{l}12 \\
(23 \%)\end{array}$ & $\begin{array}{l}1 \\
(8.33 \%)\end{array}$ & - & - \\
\hline Chen, Jiang & 135 & $\begin{array}{l}\text { NA } \\
\text { (NA\%) }\end{array}$ & - & - & - & - & - & - & $\begin{array}{l}31 \\
(22 \%)\end{array}$ & - & - & $4(12 . !$ \\
\hline Heili-Frades & 4712 & $\begin{array}{l}4086 \\
(86 \%)\end{array}$ & $\begin{array}{l}210 \\
(5.14 \%)\end{array}$ & $\begin{array}{l}659 \\
(16.13 \%)\end{array}$ & - & - & 3217 (78.73\%) & - & $\begin{array}{l}626 \\
(13 \%)\end{array}$ & $\begin{array}{l}23 \\
(3.67 \%)\end{array}$ & $\begin{array}{l}161 \\
(25.72 \%)\end{array}$ & - \\
\hline Kim, Garg & 2490 & $\begin{array}{l}2070 \\
(83 \%)\end{array}$ & $\begin{array}{l}128 \\
(6.18 \%)\end{array}$ & $\begin{array}{l}481 \\
(23.24 \%)\end{array}$ & - & - & 1461 (70.58\%) & - & $\begin{array}{l}420 \\
(16 \%)\end{array}$ & $\begin{array}{l}22 \\
(5.24 \%)\end{array}$ & $\begin{array}{l}161 \\
(38.33 \%)\end{array}$ & - \\
\hline Al-Hindawi & 31 & $\begin{array}{l}15 \\
(48 \%)\end{array}$ & $\begin{array}{l}0 \\
(0.00 \%)\end{array}$ & $\begin{array}{l}10 \\
(66.67 \%)\end{array}$ & - & $\begin{array}{l}5 \\
(33.33 \%)\end{array}$ & - & - & $\begin{array}{l}16 \\
(51 \%)\end{array}$ & $\begin{array}{l}1 \\
(6.25 \%)\end{array}$ & $\begin{array}{l}12 \\
(75.00 \%)\end{array}$ & - \\
\hline Louis & 22 & $\begin{array}{l}16 \\
(72 \%)\end{array}$ & - & - & 7 (43.75\%) & - & - & $\begin{array}{l}9 \\
(56.25 \%)\end{array}$ & $\begin{array}{l}6 \\
(27 \%)\end{array}$ & - & - & $3(50.1$ \\
\hline Soto-Mota & 400 & $\begin{array}{l}200 \\
(50 \%)\end{array}$ & - & - & $23(11.50 \%)$ & - & - & $\begin{array}{l}177 \\
(88.50 \%)\end{array}$ & $\begin{array}{l}200 \\
(50 \%)\end{array}$ & - & - & $25(12$ \\
\hline \multirow[t]{2}{*}{ Garibaldi } & 747 & $\begin{array}{l}634 \\
(84 \%)\end{array}$ & $\begin{array}{l}36 \\
(5.68 \%)\end{array}$ & $\begin{array}{l}129 \\
(20.35 \%)\end{array}$ & - & - & - & $\begin{array}{l}469 \\
(73.97 \%)\end{array}$ & $\begin{array}{l}113 \\
(15 \%)\end{array}$ & $\begin{array}{l}6 \\
(5.31 \%)\end{array}$ & $\begin{array}{l}36 \\
(31.86 \%)\end{array}$ & - \\
\hline & & 2100 & 270 & 1220 & & 1170 & & 1218 & 51ak & 011 & $125 n$ & \\
\hline
\end{tabular}




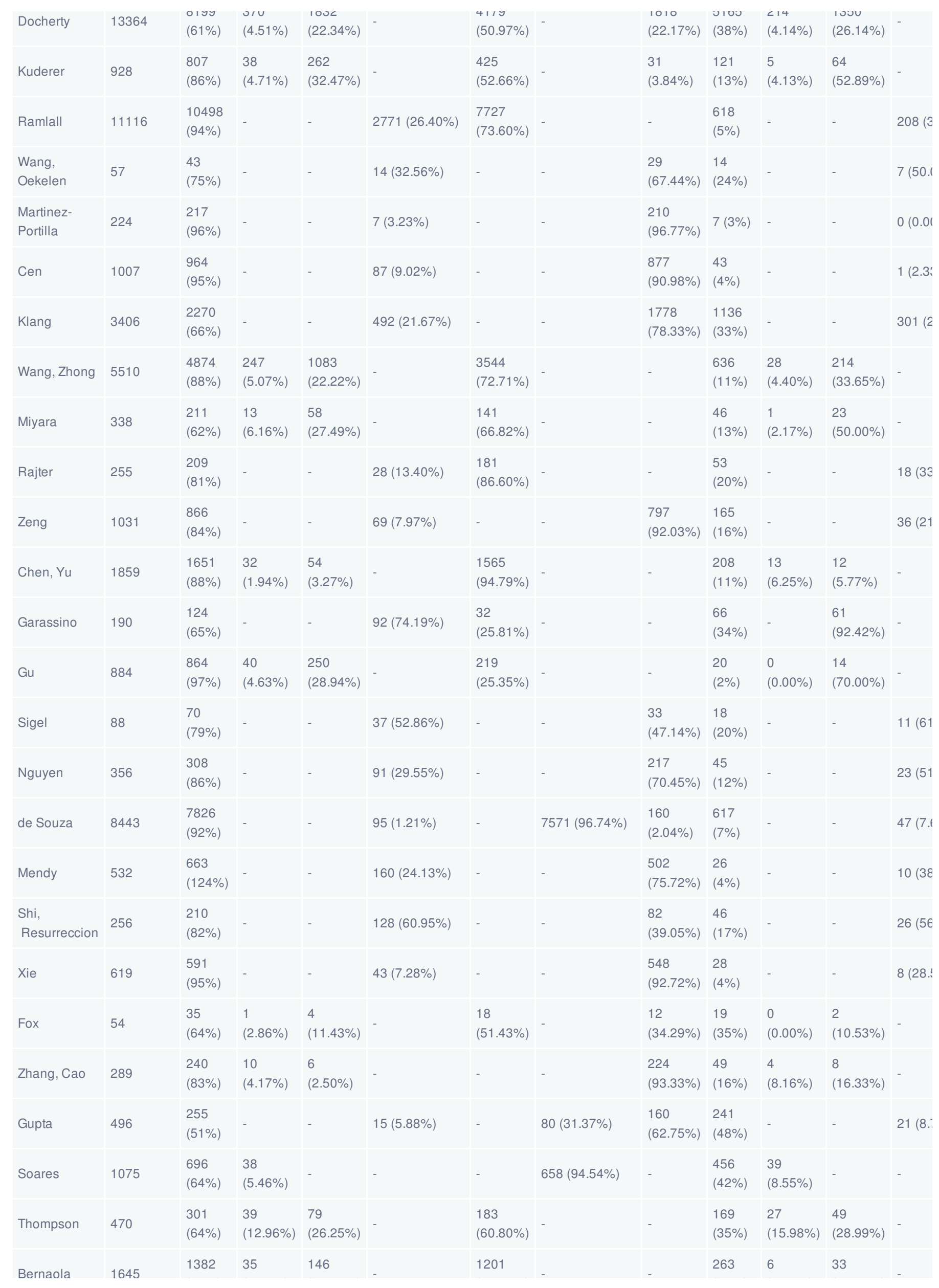




\begin{tabular}{|c|c|c|c|c|c|c|c|c|c|c|c|c|}
\hline & & $(84 \%)$ & $(2.53 \%)$ & $(10.56 \%)$ & & $(86.90 \%)$ & & & $(15 \%)$ & $(2.28 \%)$ & $(12.55 \%)$ & \\
\hline Islam & 654 & $\begin{array}{l}631 \\
(96 \%)\end{array}$ & $\begin{array}{l}103 \\
(16.32 \%)\end{array}$ & - & - & - & - & $\begin{array}{l}507 \\
(80.35 \%)\end{array}$ & $\begin{array}{l}23 \\
(3 \%)\end{array}$ & $\begin{array}{l}3 \\
(13.04 \%)\end{array}$ & - & - \\
\hline Philipose & 466 & $\begin{array}{l}267 \\
(57 \%)\end{array}$ & $\begin{array}{l}19 \\
(7.12 \%)\end{array}$ & $\begin{array}{l}204 \\
(76.40 \%)\end{array}$ & - & $\begin{array}{l}44 \\
(16.48 \%)\end{array}$ & - & - & $\begin{array}{l}199 \\
(42 \%)\end{array}$ & $\begin{array}{l}9 \\
(4.52 \%)\end{array}$ & $\begin{array}{l}137 \\
(68.84 \%)\end{array}$ & - \\
\hline Dashti & 4140 & $\begin{array}{l}3953 \\
(95 \%)\end{array}$ & - & - & $1068(27.02 \%)$ & $\begin{array}{l}2078 \\
(52.57 \%)\end{array}$ & - & $\begin{array}{l}804 \\
(20.34 \%)\end{array}$ & $\begin{array}{l}187 \\
(4 \%)\end{array}$ & - & - & 109 \\
\hline Fillmore & 1794 & $\begin{array}{l}1566 \\
(87 \%)\end{array}$ & $\begin{array}{l}408 \\
(26.05 \%)\end{array}$ & $\begin{array}{l}758 \\
(48.40 \%)\end{array}$ & - & $\begin{array}{l}279 \\
(17.82 \%)\end{array}$ & - & $\begin{array}{l}98 \\
(6.26 \%)\end{array}$ & $\begin{array}{l}228 \\
(12 \%)\end{array}$ & $\begin{array}{l}44 \\
(19.30 \%)\end{array}$ & $\begin{array}{l}141 \\
(61.84 \%)\end{array}$ & - \\
\hline Pan & 3536 & $\begin{array}{l}3302 \\
(93 \%)\end{array}$ & - & - & $862(26.11 \%)$ & - & - & $\begin{array}{l}2440 \\
(73.89 \%)\end{array}$ & $\begin{array}{l}234 \\
(6 \%)\end{array}$ & - & - & $82(35$ \\
\hline Zhao, Chen & 474 & $\begin{array}{l}398 \\
(83 \%)\end{array}$ & $\begin{array}{l}87 \\
(21.86 \%)\end{array}$ & - & - & - & - & $\begin{array}{l}311 \\
(78.14 \%)\end{array}$ & $\begin{array}{l}82 \\
(17 \%)\end{array}$ & $\begin{array}{l}36 \\
(43.90 \%)\end{array}$ & - & - \\
\hline Holman & 10989 & $\begin{array}{l}\text { NA } \\
\text { (NA\%) }\end{array}$ & - & - & - & - & - & - & $\begin{array}{l}10989 \\
(100 \%)\end{array}$ & $\begin{array}{l}609 \\
(5.54 \%)\end{array}$ & $\begin{array}{l}4684 \\
(42.62 \%)\end{array}$ & - \\
\hline Chand & 300 & $\begin{array}{l}143 \\
(47 \%)\end{array}$ & $\begin{array}{l}23 \\
(16.08 \%)\end{array}$ & - & - & - & - & $\begin{array}{l}120 \\
(83.92 \%)\end{array}$ & $\begin{array}{l}157 \\
(52 \%)\end{array}$ & $\begin{array}{l}44 \\
(28.03 \%)\end{array}$ & - & - \\
\hline Oliveira & 131 & $\begin{array}{l}105 \\
(80 \%)\end{array}$ & - & - & $16(15.24 \%)$ & - & $83(79.05 \%)$ & $\begin{array}{l}6 \\
(5.71 \%)\end{array}$ & $\begin{array}{l}26 \\
(19 \%)\end{array}$ & - & - & 7 (26.) \\
\hline Vilar-Garcia & 328892 & $\begin{array}{l}316605 \\
(96 \%)\end{array}$ & $\begin{array}{l}71215 \\
(22.49 \%)\end{array}$ & - & - & - & - & $\begin{array}{l}245390 \\
(77.51 \%)\end{array}$ & $\begin{array}{l}12287 \\
(3 \%)\end{array}$ & $\begin{array}{l}3103 \\
(25.25 \%)\end{array}$ & - & - \\
\hline Ibarra-Nava & 416546 & $\begin{array}{l}370038 \\
(88 \%)\end{array}$ & $\begin{array}{l}27001 \\
(7.30 \%)\end{array}$ & - & - & - & - & $\begin{array}{l}343037 \\
(92.70 \%)\end{array}$ & $\begin{array}{l}46508 \\
(11 \%)\end{array}$ & $\begin{array}{l}3817 \\
(8.21 \%)\end{array}$ & - & - \\
\hline Rubio-Rivas & 186 & $\begin{array}{l}147 \\
(79 \%)\end{array}$ & $\begin{array}{l}7 \\
(4.76 \%)\end{array}$ & $\begin{array}{l}32 \\
(21.77 \%)\end{array}$ & - & $\begin{array}{l}108 \\
(73.47 \%)\end{array}$ & - & - & $\begin{array}{l}39 \\
(20 \%)\end{array}$ & $\begin{array}{l}1 \\
(2.56 \%)\end{array}$ & $\begin{array}{l}6 \\
(15.38 \%)\end{array}$ & - \\
\hline Ren & 432 & $\begin{array}{l}289 \\
(66 \%)\end{array}$ & $\begin{array}{l}25 \\
(8.65 \%)\end{array}$ & - & - & $\begin{array}{l}264 \\
(91.35 \%)\end{array}$ & - & - & $\begin{array}{l}143 \\
(33 \%)\end{array}$ & $\begin{array}{l}18 \\
(12.59 \%)\end{array}$ & - & 125 (8 \\
\hline Ullah & 212 & $\begin{array}{l}158 \\
(74 \%)\end{array}$ & $\begin{array}{l}22 \\
(13.92 \%)\end{array}$ & $\begin{array}{l}67 \\
(42.41 \%)\end{array}$ & - & $\begin{array}{l}63 \\
(39.87 \%)\end{array}$ & - & $\begin{array}{l}6 \\
(3.80 \%)\end{array}$ & $\begin{array}{l}54 \\
(25 \%)\end{array}$ & $\begin{array}{l}2 \\
(3.70 \%)\end{array}$ & $\begin{array}{l}35 \\
(64.81 \%)\end{array}$ & - \\
\hline Dashti & 3401 & $\begin{array}{l}2892 \\
(85 \%)\end{array}$ & $\begin{array}{l}190 \\
(6.57 \%)\end{array}$ & $\begin{array}{l}689 \\
(23.82 \%)\end{array}$ & - & $\begin{array}{l}1756 \\
(60.72 \%)\end{array}$ & - & $\begin{array}{l}257 \\
(8.89 \%)\end{array}$ & $\begin{array}{l}509 \\
(14 \%)\end{array}$ & $\begin{array}{l}20 \\
(3.93 \%)\end{array}$ & $\begin{array}{l}171 \\
(33.60 \%)\end{array}$ & - \\
\hline Nicholson & 1040 & $\begin{array}{l}829 \\
(79 \%)\end{array}$ & $\begin{array}{l}70 \\
(8.44 \%)\end{array}$ & $\begin{array}{l}163 \\
(19.66 \%)\end{array}$ & - & $\begin{array}{l}320 \\
(38.60 \%)\end{array}$ & - & $\begin{array}{l}276 \\
(33.29 \%)\end{array}$ & $\begin{array}{l}211 \\
(20 \%)\end{array}$ & $\begin{array}{l}16 \\
(7.58 \%)\end{array}$ & $\begin{array}{l}68 \\
(32.23 \%)\end{array}$ & - \\
\hline Kalan & 193 & $\begin{array}{l}188 \\
(97 \%)\end{array}$ & $\begin{array}{l}14 \\
(7.45 \%)\end{array}$ & - & - & $\begin{array}{l}162 \\
(86.17 \%)\end{array}$ & - & $\begin{array}{l}12 \\
(6.38 \%)\end{array}$ & $5(2 \%)$ & $\begin{array}{l}0 \\
(0.00 \%)\end{array}$ & - & - \\
\hline 1 & & & & & & & & & & & & 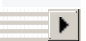 \\
\hline
\end{tabular}

Note. Solis et al. and the OpenSAFELY Collaborative reported on mortality by smoking status in a multivariable analysis but did not present raw data for both the exposure and outcome variables. 


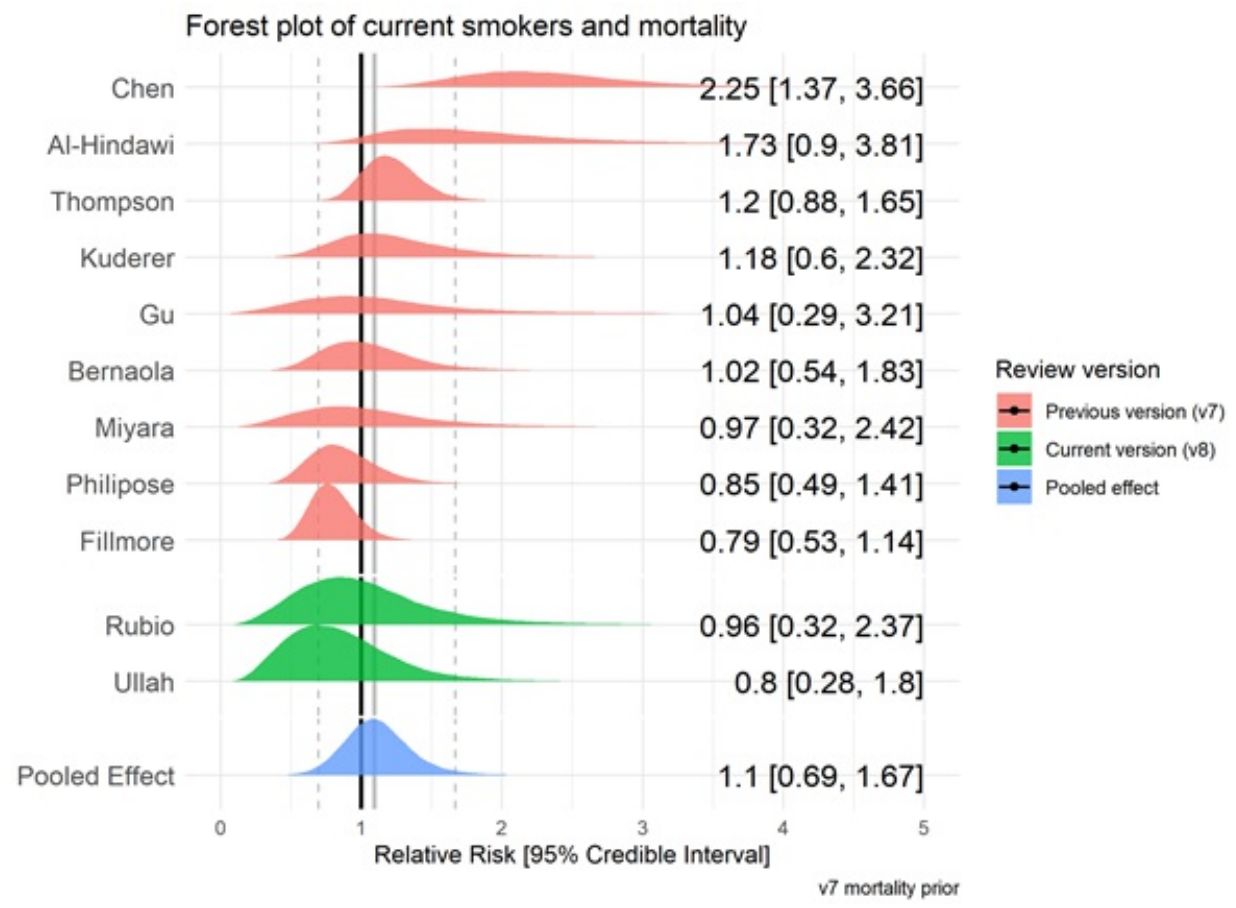

Figure 9. Forest plot for the risk of mortality in current vs. never smokers. Prior from previous version (v7) RR $=1.22$

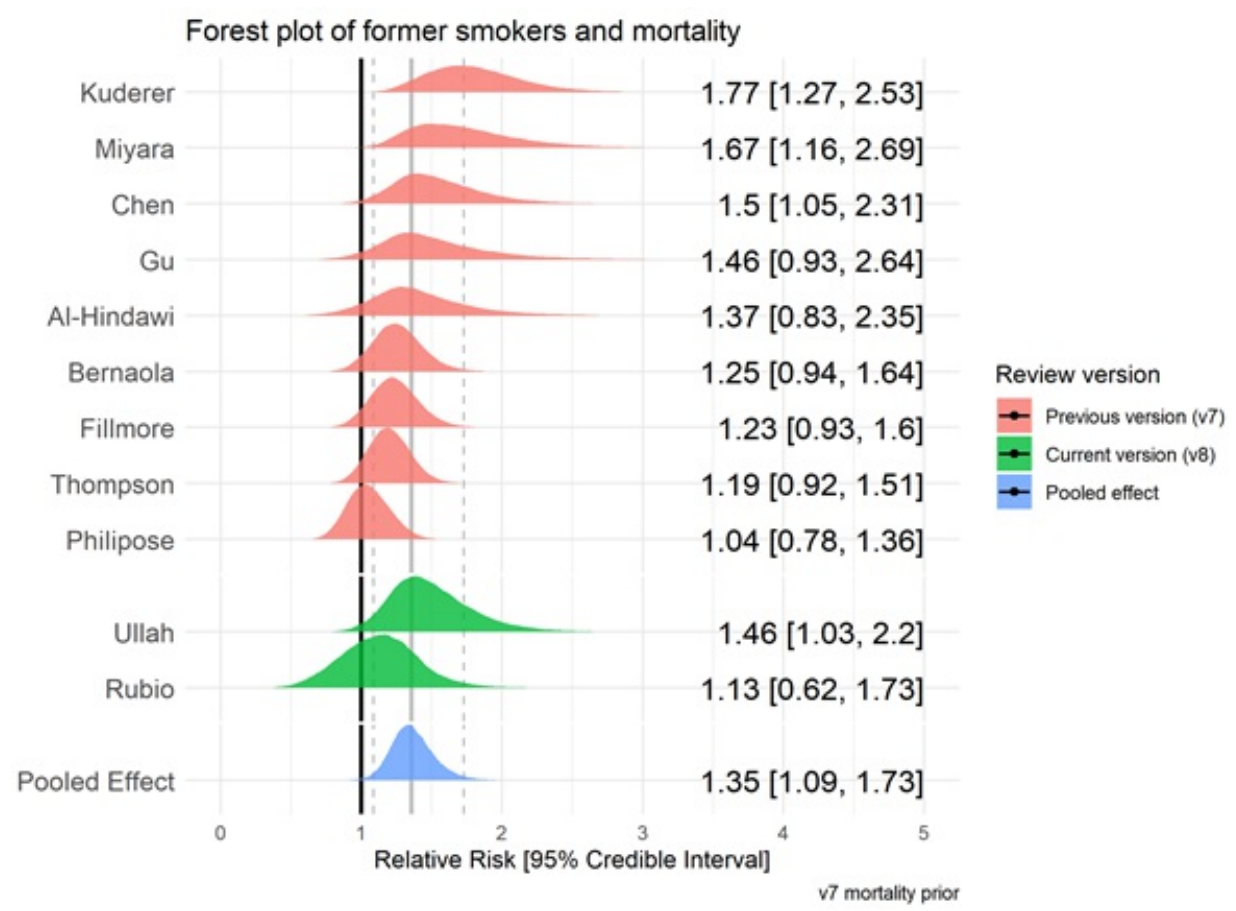

Figure 10. Forest plot for the risk of mortality in former smokers vs. never smokers. Prior from previous version (v7) RR $=1.39$

\section{Discussion}

This living rapid review found uncertainty in the majority of 256 studies arising from the recording of smoking status. Notwithstanding these uncertainties, compared with overall adult national prevalence estimates, recorded current smoking 
rates in most studies were lower than expected. In a subset of better-quality studies ( $n=19)$, current but not former smokers had a reduced risk of testing positive for SARS-CoV-2 but current smokers appeared somewhat more likely to present for testing and/or receive a test. Data for current smokers on the risk of hospitalisation, disease severity and mortality were inconclusive, and favoured there being no important associations with hospitalisation and mortality and a small but important increase in the risk of severe disease. Former smokers were at increased risk of hospitalisation, disease severity and mortality compared with never smokers.

\section{Issues complicating interpretation}

Interpretation of results from studies conducted during the first phase of the SARS-CoV-2 pandemic is complicated by several factors (see Figure 11):

1) Exposure to SARS-CoV-2 is heterogeneous with different subgroups at heightened risk of infection at different stages of the pandemic. This will likely introduce bias in studies assessing the rate of infection by smoking status conducted early on.

2) Current and former smokers may be more likely to meet local criteria for community testing due to increased prevalence of symptoms consistent with SARS-CoV-2 infection, such as cough, increased sputum production or altered sense of smell or taste ${ }^{35}$. Evidence from a small number of studies indicates that current smokers may be more likely to present for testing, hence increasing the denominator in comparisons with never smokers and potentially inflating the rate of negative tests in current smokers. Infection positivity rates estimated among random samples are more informative. We identified one population study conducted in Hungary reporting on seroprevalence and smoking status ${ }^{36}$; however, the response rate was fairly low at $58.8 \%$ and the current smoking rate was 10 percentage points below national prevalence estimates, thus questioning the representativeness of the final sample. Similarly, a second representative population survey with results from three regions in France ${ }^{37}$ reported a current smoking rate of more than 10 percentage points below national prevalence $(12 \% \text { vs. } 25 \% \text { daily smoking prevalence })^{38}$. Smoking status is being collected in at least two large representative infection and antibody surveys in the $\mathrm{UK}^{39,40}$; however, results stratified by smoking status have not yet been reported.

3) Testing for acute infection requires swabbing of the mucosal epithelium, which may be disrupted in current smokers, potentially altering the sensitivity of assays ${ }^{41}$.

4) Heated and humidified air may act to disrupt the ability of the virus to persist in the airway mucosa of smokers. There is some evidence that transient localised hyperthermia can inhibit replication of rhinoviruses, a non-enveloped virus that causes the common cold ${ }^{42}$. However, as SARS-CoV-2 is an enveloped virus ${ }^{43}$, it is unclear whether a similar protective effect against viral replication or invasion by heated and humidified air may occur.

5) Diagnostic criteria for SARS-CoV-2 infection and COVID-19 have changed during the course of the pandemic ${ }^{44}$. It was not possible to extract details on the specific RT-PCR technique or platforms used across the included studies due to reporting gaps. Different platforms have varying sensitivity and specificity to detect SARS-CoV-2 infection.

6) Most included studies relied on EHRs as the source of information on smoking status. Research shows large discrepancies between EHRs and actual behaviour ${ }^{45}$. Known failings of EHRs include implausible longitudinal changes, 
such as former smokers being recorded as never smokers at subsequent hospital visits ${ }^{45}$. Misreporting on the part of the patient (perhaps due to perceived stigmatisation) has also been observed, with biochemical measures showing higher rates of smoking compared with self-report in hospitalised patients in the US ${ }^{46}$. It is hence possible that under-reporting of current and former smoking status in hospitals occurred across the included studies.

7) Individuals with severe COVID-19 symptoms may have stopped smoking immediately before admission to hospital and may therefore not have been recorded as current smokers (i.e. reverse causality).

8) Smokers with COVID-19 may be less likely to receive a SARS-CoV-2 test or present to hospital due to lack of access to healthcare and may be more likely to die in the community from sudden complications (i.e. self-selection bias) and thus not be recorded.

9) If there is a protective effect of nicotine on COVID-19 disease outcomes, abrupt nicotine withdrawal upon hospitalisation may lead to worse outcomes ${ }^{12}$.

10) During periods of heightened demand of limited healthcare resources, current and former smokers with extensive comorbidities may have reduced priority for intensive care admission, thus leading to higher in-hospital mortality.

11) Given lack of knowledge of the disease progression and long-term outcomes of COVID-19, it is unclear whether studies conducted thus far in the pandemic have monitored patients for a sufficient time period to report complete survival outcomes or whether they are subject to early censoring.

12) Reasons for hospitalisation vary by country and time in the pandemic. For example, early cases may have been hospitalised for isolation and quarantine reasons and not due to medical necessity. It is plausible this may have skewed early data towards less severe cases. In addition, the observed association between former smoking and greater disease severity may be explained by collider bias ${ }^{47}$, where conditioning on a collider (e.g. testing or hospitalisation) by design or analysis may introduce a spurious association between current or former smoking (a potential cause of testing or hospitalisation) and SARS-CoV-2 infection/adverse outcomes from COVID-19 (potentially exacerbated by smoking) ${ }^{48}$. 13) The majority of included studies were conducted in hospital settings. It is plausible that a non-trivial proportion of patients were infected with SARS-CoV-2 while being an inpatient for a different medical reason. If so, this may have biased the hospitalised populations towards older and more frail groups, who are less likely to be smokers ${ }^{49}$.

Figure 11. A schematic of some of the interpretation issues for the association of smoking and SARS-CoV-2/COVID-19. * Indicates potential confounding with smoking status.

\section{Limitations}

This living rapid evidence review was limited by having a single reviewer extracting data with a second independently verifying the data extracted to minimise errors, restricting the search to one electronic database and one pre-print server and by not including at least three large population surveys due to their reliance on self-reported suspected or confirmed SARS-CoV-2 infection (which means they do not meet our eligibility criteria) ${ }^{35,50,51}$. We also did not include a large, UKbased, representative seroprevalence study ${ }^{52}$ in our meta-analyses as the odds of testing positive in former smokers was 
not reported. However, the odds of infection for current smokers $(\mathrm{OR}=0.64,95 \% \mathrm{Cl}=0.58-0.71)$ was in concordance with the pooled estimate in our meta-analysis. Population surveys - particularly with linked data on confirmed infection or antibodies - will be included in future review versions to help mitigate some of the limitations of healthcare based observational studies. The comparisons of current and former smoking prevalence in the included studies with national prevalence estimates did not adjust observed prevalence for the demographic profile of those tested/admitted to hospital. Other reviews focused on this comparison have applied adjustments for sex and age, and continue to find lower than expected prevalence - notwithstanding the issues complicating interpretation described above ${ }^{17}$.

\section{Implications for research, policy and practice}

Further scientific research is needed to resolve the mixed findings summarised in our review. First, clinical trials of the posited therapeutic effect of nicotine could have important implications both for smokers and for improved understanding of how the SARS-CoV-2 virus causes disease in humans. Such trials should focus on medicinal nicotine (as smoked tobacco is a dirty delivery mechanism that could mask beneficial effects) and potentially differentiate between different modes of delivery (i.e. inhaled vs. ingested) since this can affect pharmacokinetics ${ }^{53}$ and potential therapeutic effects. A second research priority would be a large, representative (randomly sampled) population survey with a validated assessment of smoking status which distinguishes between recent and long-term ex-smokers - ideally biochemically verified - and assesses seroprevalence and links to health records.

In the meantime, public-facing messages about the possible protective effect of smoking or nicotine are premature. In our view, until there is further research, the quality of the evidence does not justify the huge risk associated with a message likely to reach millions of people that a lethal activity, such as smoking, may protect against COVID-19. It continues to be appropriate to recommend smoking cessation and emphasise the role of alternative nicotine products to support smokers to stop as part of public health efforts during COVID-19. At the very least, smoking cessation reduces acute risks from cardiovascular disease and could reduce demands on the healthcare system ${ }^{54}$. GPs and other healthcare providers can play a crucial role - brief, high-quality and free online training is available at National Centre for Smoking Cessation and Training.

\section{Conclusion}

Across 256 studies, recorded smoking prevalence was generally lower than national prevalence estimates. Current smokers were at reduced risk of testing positive for SARS-CoV-2 and former smokers were at increased risk of hospitalisation, disease severity and mortality compared with never smokers.

\section{Acknowledgements}

An original short review for the Royal College of Physicians was converted to an extended living review after a request 
by Martin Dockrell, Tobacco Control Lead, Public Health England. All scientific decisions were made by the authors independently of funders and external organisations. The authors would like to thank Rosemary Koper for her assistance in running the electronic searches and data extraction.

\section{Declaration of conflicts of interest}

DS and OP have no conflicts of interest to declare. LS has received a research grant and honoraria for a talk and travel expenses from manufacturers of smoking cessation medications (Pfizer and Johnson \& Johnson). JB has received unrestricted research funding to study smoking cessation from companies who manufacture smoking cessation medications. All authors declare no financial links with tobacco companies or e-cigarette manufacturers or their representatives.

\section{Funding statement}

DS is supported by a PhD studentship from the UK Biotechnology and Biological Sciences Research Council [BB/M009513/1]. OP receives salary support from Cancer Research UK (C1417/A22962). JB, LS, \& OP are members of SPECTRUM, a UK Prevention Research Partnership Consortium (MR/S037519/1). UKPRP is an initiative funded by the UK Research and Innovation Councils, the Department of Health and Social Care (England) and the UK devolved administrations, and leading health research charities.

\section{Future review versions}

https://www.qeios.com/read/latest-UJR2AW

\section{Previous review versions}

Version 1: https://doi.org/10.32388/UJR2AW

Version 2: https://doi.org/10.32388/UJR2AW.3

Version 3: https://doi.org/10.32388/UJR2AW.4

Version 4: https://doi.org/10.32388/UJR2AW.5

Version 5: https://doi.org/10.32388/UJR2AW.6

Version 6: https://doi.org/10.32388/UJR2AW.7

Version 7: https://doi.org/10.1111/add.15276

\section{Data availability}

All data contributing to the current and future review versions are available here: https://doi.org/10.6084/m9.figshare.12756020

All code required to reproduce the current and future analyses are available here: https://doi.org/10.5281/zenodo.4002046

\section{References}


1 Guan W, Ni Z, Hu YY, et al. Clinical Characteristics of Coronavirus Disease 2019 in China. N Engl J Med 2020; : NEJMoa2002032.

2 Hoffmann M, Kleine-Weber H, Schroeder S, et al. SARS-CoV-2 Cell Entry Depends on ACE2 and TMPRSS2 and Is Blocked by a Clinically Proven Protease Inhibitor. Cell 2020; published online March 5. DOI:10.1016/j.cell.2020.02.052. 3 Brake SJ, Barnsley K, Lu W, McAlinden KD, Eapen MS, Sohal SS. Smoking Upregulates Angiotensin-Converting Enzyme-2 Receptor: A Potential Adhesion Site for Novel Coronavirus SARS-CoV-2 (Covid-19). J Clin Med 2020 Vol 9 Page 841 2020; 9: 841.

4 Cai G. Bulk and Single-Cell Transcriptomics Identify Tobacco-Use Disparity in Lung Gene Expression of ACE2, the Receptor of 2019-nCov. 2020; published online March 2. DOI:10.20944/PREPRINTS202002.0051.V3.

5 Oakes JM, Fuchs RM, Gardner JD, Lazartigues E, Yue X. Nicotine and the renin-angiotensin system. Am. J. Physiol. - Regul. Integr. Comp. Physiol. 2018; 315: R895-906.

6 Denholm JT, Gordon CL, Johnson PD, et al. Hospitalised adult patients with pandemic (H1N1) 2009 influenza in Melbourne, Australia. Med J Aust 2010; 192: 84-6.

7 Abadom TR, Smith AD, Tempia S, Madhi SA, Cohen C, Cohen AL. Risk factors associated with hospitalisation for influenza-associated severe acute respiratory illness in South Africa: A case-population study. Vaccine 2016; 34: 5649_ 55.

8 Almirall J, González CA, Balanzó X, Bolíbar I. Proportion of community-acquired pneumonia cases attributable to tobacco smoking. Chest 1999; 116: 375-9.

9 Feldman C, Anderson R. Cigarette smoking and mechanisms of susceptibility to infections of the respiratory tract and other organ systems. J. Infect. 2013; 67: 169-84.

10 Dye JA, Adler KB. Occasional review Effects of cigarette smoke on epithelial cells of the respiratory tract. Thorax 1994; 49: 825-34.

11 Vardavas CI, Nikitara K. COVID-19 and smoking: A systematic review of the evidence. Tob Induc Dis 2020; 18: 20.

12 Farsalinos K, Niaura R, Le Houezec J, et al. Editorial: Nicotine and SARS-CoV-2: COVID-19 may be a disease of the nicotinic cholinergic system. Toxicol Rep 2020; published online April. DOI:10.1016/j.toxrep.2020.04.012.

13 Emami A, Javanmardi F, Pirbonyeh N, Akbari A. Prevalence of Underlying Diseases in Hospitalized Patients with COVID-19: a Systematic Review and Meta-Analysis. Arch Acad Emerg Med 2020; 8: e35.

14 Alqahtani JS, Oyelade T, Aldhahir AM, et al. Prevalence, Severity and Mortality associated with COPD and Smoking in patients with COVID-19: A Rapid Systematic Review and Meta-Analysis. medRxiv 2020; : 2020.03.25.20043745.

15 Patanavanich R, Glantz SA. Smoking is Associated with COVID-19 Progression: A Meta-Analysis. medRxiv 2020. DOI:10.14171/j.2095-5944.sg.2014.02.004.

16 Berlin I, Thomas D, Le Faou A-L, Cornuz J. COVID-19 and Smoking. Nicotine Tob Res DOI:10.1093/NTR/NTAA059.

17 Farsalinos K, Barbouni A, Niaura R. Systematic review of the prevalence of current smoking among hospitalized COVID-19 patients in China: could nicotine be a therapeutic option? Intern Emerg Med 2020; published online May 9. DOI:10.1007/s11739-020-02355-7. 
18 Grundy* EJ, Suddek* T, Filippidis FT, Majeed A, Coronini-Cronberg S. Smoking, SARS-CoV-2 and COVID-19: A review of reviews considering implications for public health policy and practice. Tob Induc Dis 2020; 18. DOI:10.18332/tid/124788.

19 Elliott JH, Turner T, Clavisi O, et al. Living Systematic Reviews: An Emerging Opportunity to Narrow the EvidencePractice Gap. PLoS Med 2014; 11. DOl:10.1371/journal.pmed.1001603.

20 Tricco AC, Antony J, Zarin W, et al. A scoping review of rapid review methods. BMC Med 2015; $13: 224$.

21 Simons D, Brown J, Shahab L, Perski O. Smoking and COVID-19: Rapid evidence review for the Royal College of Physicians, London (UK). Qeios 2020; published online April 1. DOI:10.32388/VGJCUN.

22 Simons D, Shahab L, Brown J, Perski O. The association of smoking status with SARS-CoV-2 infection, hospitalisation and mortality from COVID-19: A living rapid evidence review with Bayesian meta-analyses (version 7). Addiction; n/a. DOI:10.1111/add.15276.

23 R Core Team. The R Project for Statistical Computing. 2013; : 1-12.

24 Bürkner P-C. Advanced Bayesian Multilevel Modeling with the R Package brms. ArXiv170511123 Stat2017; published online Oct 15. http://arxiv.org/abs/1705.11123 (accessed July 26, 2020).

25 Simons D, Shahab L, Brown J, Perski O. The association of smoking status with SARS-CoV-2 infection, hospitalisation and mortality from COVID-19: A living rapid evidence review (version 5). Qeios 2020; published online July 1. DOI:10.32388/UJR2AW.6.

26 Miyara M, Tubach F, Martinez V, et al. Low rate of daily smokers in patients with symptomatic COVID-19. medrxiv 2020; : 2020.06.10.20127514.

27 Rimland CA, Morgan CE, Bell GJ, et al. Clinical characteristics and early outcomes in patients with COVID-19 treated with tocilizumab at a United States academic center. medRxiv 2020; : 2020.05.13.20100404.

28 Yannick Girardeau, Yoan Gallous, Guillaume de Bonnecaze, et al. Confirmed central olfactory system lesions on brain MRI in COVID-19 patients with anosmia: a case-series | medRxiv. https://doi.org/10.1101/2020.07.08.20148692 (accessed Aug 25, 2020).

29 Ebinger J, Botwin GJ, Albert CM, et al. SARS-CoV-2 Seroprevalence Across a Diverse Cohort of Healthcare Workers. medRxiv 2020; : 2020.07.31.20163055.

30 Islam MZ, Riaz BK, Islam AS, et al. Risk factors associated with morbidity and mortality outcomes of COVID-19 patients on the 14th and 28th day of the disease course: a retrospective cohort study in Bangladesh. medRxiv 2020; : 2020.08.17.20176586.

31 Rentsch CT, Kidwai-Khan F, Tate JP, et al. Covid-19 Testing, Hospital Admission, and Intensive Care Among 2,026,227 United States Veterans Aged 54-75 Years. medRxiv 2020; : 2020.04.09.20059964.

32 Odani S. Tobacco Product Use Among Military Veterans — United States, 2010-2015. MMWR Morb Mortal Wkly Rep 2018; 67. DOI:10.15585/mmwr.mm6701a2.

33 Niedzwiedz CL, O'Donnell CA, Jani BD, et al. Ethnic and socioeconomic differences in SARS-CoV-2 infection: prospective cohort study using UK Biobank. BMC Med 2020; 18: 160.

34 Trubiano JA, Vogrin S, Smibert OC, et al. COVID-MATCH65 - A prospectively derived clinical decision rule for severe acute respiratory syndrome coronavirus 2. medRxiv 2020; : 2020.06.30.20143818. 
Hopkinson NS, Rossi NN, Moustafa JE-SSE, et al. Current tobacco smoking and risk from COVID-19 results from a population symptom app in over 2.4 million people. medrxiv 2020; 44: 2020.05.18.20105288.

36 Merkely B, Szabó AJ, Kosztin A, et al. Novel coronavirus epidemic in the Hungarian population, a cross-sectional nationwide survey to support the exit policy in Hungary. GeroScience 2020; published online July 17.

DOI:10.1007/s11357-020-00226-9.

37 Carrat F, Lamballerie X de, Rahib D, et al. Seroprevalence of SARS-CoV-2 among adults in three regions of France following the lockdown and associated risk factors: a multicohort study. medRxiv 2020; : 2020.09.16.20195693.

38 Andler R. BAISSE DE LA PRÉVALENCE DU TABAGISME QUOTIDIEN PARMI LES ADULTES : RÉSULTATS DU BAROMĖTRE DE SANTÉ PUBLIQUE FRANCE 2018 / REDUCTION OF DAILY SMOKING RATE AMONG ADULTS: RESULTS FROM THE 2018 SANTÉ PUBLIQUE FRANCE HEALTH BAROMETER. $2019 ;: 7$.

39 Major home testing programme for coronavirus will track levels of infection in the community - GOV.UK.

https://www.gov.uk/government/news/major-home-testing-programme-for-coronavirus-will-track-levels-of-infection-in-thecommunity (accessed May 22, 2020).

40 COVID-19 Infection Survey (CIS) - Office for National Statistics.

https://www.ons.gov.uk/surveys/informationforhouseholdsandindividuals/householdandindividualsurveys/covid19infection surveycis (accessed June 30, 2020).

41 de Lusignan S, Dorward J, Correa A, et al. Risk factors for SARS-CoV-2 among patients in the Oxford Royal College of General Practitioners Research and Surveillance Centre primary care network: a cross-sectional study. Lancet Infect Dis 2020; 0. DOI:10.1016/S1473-3099(20)30371-6.

42 Conti C, de Marco A, Mastromarino P, Tomao P, Santoro MG. Antiviral Effect of Hyperthermic Treatment in Rhinovirus Infection. Antimicrob Agents Chemother 1999; 43: 822-9.

43 Schoeman D, Fielding BC. Coronavirus envelope protein: current knowledge. Virol J 2019; 16: 69.

44 World Health Organisation. Laboratory testing for 2019 novel coronavirus (2019-nCoV) in suspected human cases. https://www.who.int/publications-detail-redirect/10665-331501 (accessed July 29, 2020).

45 Polubriaginof F, Salmasian H, Albert DA, Vawdrey DK. Challenges with Collecting Smoking Status in Electronic Health Records. AMIA Annu Symp Proc AMIA Symp 2017; 2017: 1392-400.

46 Benowitz NL, Schultz KE, Haller CA, Wu AHB, Dains KM, Jacob P. Prevalence of smoking assessed biochemically in an urban public hospital: a rationale for routine cotinine screening. Am J Epidemiol 2009; 170: 885-91.

47 Griffith G, Morris TT, Tudball M, et al. Collider bias undermines our understanding of COVID-19 disease risk and severity. medRxiv 2020; : 2020.05.04.20090506.

48 Murray E. Causation in smoking and COVID-19. Twitter. 2020.

https://twitter.com/EpiEllie/status/1258607277357006849?s=20.

49 Mangera Z, Lewis A, Hutchinson J, Searle L, Agrawal S. Smoking prevalence in UK hospital admissions from a national observational study. Eur Respir J 2017; 50. DOl:10.1183/1393003.congress-2017.PA1268.

50 Bowyer RCE, Varsavsky T, Carole H. Geo-social gradients in predicted COVID-19 prevalence and severity in Great Britain : results from Affiliations : Corresponding authors : Understanding the geographical distribution of COVID-19 through the general population is key to the provision of ade. 2020. 
51 Jackson SE, Brown J, Shahab L, Steptoe A, Fancourt D. COVID-19, smoking, and inequalities: a cross-sectional survey of adults in the UK. Submitted 2020.

52 Ward H, Atchison CJ, Whitaker M, et al. Antibody prevalence for SARS-CoV-2 in England following first peak of the pandemic: REACT2 study in 100,000 adults. medRxiv 2020; : 2020.08.12.20173690.

53 Shahab L, Brose LS, West R. Novel delivery systems for nicotine replacement therapy as an aid to smoking cessation and for harm reduction: Rationale, and evidence for advantages over existing systems. CNS Drugs 2013; 27: $1007-19$.

54 Stead LF, Buitrago D, Preciado N, Sanchez G, Hartmann-Boyce J, Lancaster T. Physician advice for smoking cessation. Cochrane Database Syst. Rev. 2013; 2017. DOI:10.1002/14651858.CD000165.pub4.

55 Guan W, Liang W, Zhao Y, et al. Comorbidity and its impact on 1590 patients with COVID-19 in China: a nationwide analysis. Eur Respir J 2020; 55. DOI:10.1183/13993003.00547-2020.

56 Lian J, Jin X, Hao S, et al. Analysis of Epidemiological and Clinical Features in Older Patients With Coronavirus Disease 2019 (COVID-19) Outside Wuhan. Clin Infect Dis DOI:10.1093/cid/ciaa242.

57 Jin X, Lian J-S, Hu J-H, et al. Epidemiological, clinical and virological characteristics of 74 cases of coronavirusinfected disease 2019 (COVID-19) with gastrointestinal symptoms. Gut 2020; 69: 1002-9.

58 Chen T, Wu D, Chen H, et al. Clinical characteristics of 113 deceased patients with coronavirus disease 2019: retrospective study. BMJ 2020; 368. DOI:10.1136/bmj.m1091.

59 Zhou F, Yu T, Du R, et al. Clinical course and risk factors for mortality of adult inpatients with COVID-19 in Wuhan, China: a retrospective cohort study. The Lancet 2020; 395: 1054-62.

60 Mo $\mathrm{P}$, Xing $\mathrm{Y}, \mathrm{Xiao} \mathrm{Y}$, et al. Clinical characteristics of refractory COVID-19 pneumonia in Wuhan, China. Clin Infect Dis DOI:10.1093/cid/ciaa270.

61 Zhang J, Dong X, Cao Y, et al. Clinical characteristics of 140 patients infected with SARS-CoV-2 in Wuhan, China. Allergy 2020; 75: 1730-41.

62 Wan S, Xiang Y, Fang W, et al. Clinical features and treatment of COVID-19 patients in northeast Chongqing. J Med Virol 2020; 92: 797-806.

63 Liu W, Tao Z-W, Wang L, et al. Analysis of factors associated with disease outcomes in hospitalized patients with 2019 novel coronavirus disease. Chin Med J (Engl) 2020; 133: 1032-1038.

64 Huang C, Wang Y, Li X, et al. Clinical features of patients infected with 2019 novel coronavirus in Wuhan, China. The Lancet 2020; 395: 497-506.

65 Zhang X, Cai H, Hu J, et al. Epidemiological, clinical characteristics of cases of SARS-CoV-2 infection with abnormal imaging findings. Int $J$ Infect Dis 2020; 94: 81-7.

66 Guo T, Fan Y, Chen M, et al. Cardiovascular Implications of Fatal Outcomes of Patients With Coronavirus Disease 2019 (COVID-19). JAMA Cardiol 2020; 5: 811-8.

67 Liu R, Ming X, Xu O, et al. Association of Cardiovascular Manifestations with In-hospital Outcomes in Patients with COVID-19: A Hospital Staff Data. medRxiv 2020; : 2020.02.29.20029348.

68 Huang Y, Yang R, Xu Y, Gong P. Clinical characteristics of 36 non-survivors with COVID-19 in Wuhan, China. medRxiv 2020; : 2020.02.27.20029009. 
$69 \mathrm{Xu} \mathrm{H}, \mathrm{Hou} \mathrm{K}, \mathrm{Xu} \mathrm{H}$, et al. Acute Myocardial Injury of Patients with Coronavirus Disease 2019. medRxiv 2020; : 2020.03.05.20031591.

70 Li J, Li S, Cai Y, et al. Epidemiological and Clinical Characteristics of 17 Hospitalized Patients with 2019 Novel Coronavirus Infections Outside Wuhan, China. medRxiv 2020; : 2020.02.11.20022053.

$71 \mathrm{Hu}$ L, Chen S, Fu Y, et al. Risk Factors Associated with Clinical Outcomes in 323 COVID-19 Patients in Wuhan, China. medRxiv 2020; : 2020.03.25.20037721.

72 Wang R, Pan M, Zhang X, et al. Epidemiological and clinical features of 125 Hospitalized Patients with COVID-19 in Fuyang, Anhui, China. Int J Infect Dis 2020; 95: 421-8.

73 CDCMMWR. Preliminary Estimates of the Prevalence of Selected Underlying Health Conditions Among Patients with Coronavirus Disease 2019 — United States, February 12-March 28, 2020. MMWR Morb Mortal Wkly Rep $2020 ; 69$. DOI:10.15585/mmwr.mm6913e2.

74 Dong X, Cao Y, Lu X, et al. Eleven faces of coronavirus disease 2019. Allergy 2020; 75: 1699-709.

75 Kim ES, Chin BS, Kang CK, et al. Clinical Course and Outcomes of Patients with Severe Acute Respiratory Syndrome Coronavirus 2 Infection: a Preliminary Report of the First 28 Patients from the Korean Cohort Study on COVID19. J Korean Med Sci 2020; 35. DOI:10.3346/jkms.2020.35.e142.

76 Shi Y, Yu X, Zhao H, Wang H, Zhao R, Sheng J. Host susceptibility to severe COVID-19 and establishment of a host risk score: findings of 487 cases outside Wuhan. Crit Care 2020; 24: 108.

77 Yang X, Yu Y, Xu J, et al. Clinical course and outcomes of critically ill patients with SARS-CoV-2 pneumonia in Wuhan, China: a single-centered, retrospective, observational study. Lancet Respir Med 2020; 8: 475-81.

78 Argenziano MG, Bruce SL, Slater CL, et al. Characterization and clinical course of 1000 patients with coronavirus disease 2019 in New York: retrospective case series. BMJ 2020; 369. DOI:10.1136/bmj.m1996.

79 Solís P, Carreňo H. COVID-19 Fatality and Comorbidity Risk Factors among Confirmed Patients in Mexico. Epidemiology, 2020 DOI:10.1101/2020.04.21.20074591.

80 Richardson S, Hirsch JS, Narasimhan M, et al. Presenting Characteristics, Comorbidities, and Outcomes Among 5700 Patients Hospitalized With COVID-19 in the New York City Area. JAMA 2020; 323: 2052-9.

81 Fontanet A, Tondeur L, Madec Y, et al. Cluster of COVID-19 in northern France: A retrospective closed cohort study. medRxiv 2020; : 2020.04.18.20071134.

82 Zheng KI, Gao F, Wang X-B, et al. Letter to the Editor: Obesity as a risk factor for greater severity of COVID-19 in patients with metabolic associated fatty liver disease. Metabolism 2020; 108: 154244.

83 Liao Y, Feng Y, Wang B, et al. Clinical Characteristics and Risk factors for developed COVID-19 patients transferring to designated hospital from Jianghan Fangcang shelter Hospital: a retrospective, observational study. medRxiv 2020; : 2020.04.21.20074724.

84 Gil-Agudo A, Rodriguez-Cola M, Jimenez-Velasco I, et al. Clinical features of coronavirus disease 2019 (COVID19) in a cohort of patients with disability due to spinal cord injury. medRxiv 2020; :2020.04.20.20072918.

85 Shi P, Ren G, Yang J, et al. Clinical characteristics of imported and second-generation COVID-19 cases outside Wuhan, China: A multicenter retrospective study. medRxiv 2020; : 2020.04.19.20071472.

86 Hadjadj J, Yatim N, Barnabei L, et al. Impaired type I interferon activity and exacerbated inflammatory responses in 
severe Covid-19 patients. medRxiv 2020; : 2020.04.19.20068015.

87 Gold JAW, Wong KK, Szablewski CM, et al. Characteristics and Clinical Outcomes of Adult Patients Hospitalized with COVID-19 - Georgia, March 2020. MMWR Morb Mortal Wkly Rep 2020; 69: 545-50.

88 Yu T, Cai S, Zheng Z, et al. Association Between Clinical Manifestations and Prognosis in Patients with COVID-19. Clin Ther 2020; 42: 964-72.

89 Zheng Y, Xiong C, Liu Y, et al. Epidemiological and clinical characteristics analysis of COVID-19 in the surrounding areas of Wuhan, Hubei Province in 2020. Pharmacol Res 2020; 157: 104821.

90 Rica R de la, Borges M, Aranda M, et al. Low albumin levels are associated with poorer outcomes in a case series of COVID-19 patients in Spain: a retrospective cohort study. medRxiv 2020; : 2020.05.07.20094987.

91 Yin R, Yang Z, Wei Y, et al. Clinical characteristics of 106 patients with neurological diseases and co-morbid coronavirus disease 2019: a retrospective study. medRxiv 2020; : 2020.04.29.20085415.

92 Shi H, Zuo Y, Yalavarthi S, et al. Neutrophil calprotectin identifies severe pulmonary disease in COVID-19. medRxiv 2020; : 2020.05.06.20093070.

93 Cho ER, Slutsky AS, Jha P. Smoking and the risk of COVID-19 infection in the UK Biobank Prospective Study. medRxiv 2020; : 2020.05.05.20092445.

94 Allenbach $\mathrm{Y}$, Saadoun D, Maalouf G, et al. Multivariable prediction model of intensive care unit transfer and death: a French prospective cohort study of COVID-19 patients. medRxiv 2020; : 2020.05.04.20090118.

95 Robilotti EV, Babady NE, Mead PA, et al. Determinants of Severity in Cancer Patients with COVID-19 IIIness. medRxiv 2020; : 2020.05.04.20086322.

96 Williamson EJ, Walker AJ, Bhaskaran K, et al. OpenSAFELY: factors associated with COVID-19 death in 17 million patients. Nature 2020; : 1-11.

97 Borobia AM, Carcas AJ, Arnalich F, et al. A Cohort of Patients with COVID-19 in a Major Teaching Hospital in Europe. J Clin Med 2020; 9: 1733.

98 Giacomelli A, Ridolfo AL, Milazzo L, et al. 30-day mortality in patients hospitalized with COVID-19 during the first wave of the Italian epidemic: A prospective cohort study. Pharmacol Res 2020; 158: 104931.

99 Shah SJ, Barish PN, Prasad PA, et al. Clinical features, diagnostics, and outcomes of patients presenting with acute respiratory illness: a comparison of patients with and without COVID-19. medRxiv 2020; : 2020.05.02.20082461. 100 Kolin DA, Kulm S, Elemento O. Clinical and Genetic Characteristics of Covid-19 Patients from UK Biobank. medRxiv 2020; : 2020.05.05.20075507.

101 Lubetzky M, Aull M, Craig-Shapiro R, et al. Kidney Allograft Recipients Diagnosed with Coronavirus Disease-2019: A Single Center Report. medRxiv 2020; : 2020.04.30.20086462.

102 Goyal P, Choi JJ, Pinheiro LC, et al. Clinical Characteristics of Covid-19 in New York City. N Engl J Med 2020; 382: 2372-4.

103 Feng Y, Ling Y, Bai T, et al. COVID-19 with Different Severities: A Multicenter Study of Clinical Features. Am J Respir Crit Care Med 2020; 201: 1380-8.

104 Yao Q, Wang P, Wang X, Qie G, Chu Y. A retrospective study of risk factors for severe acute respiratory syndrome coronavirus 2 infections in hospitalized adult patients. DOI:10.20452/pamw.15312. 
105 Sami R, Soltaninejad F, Amra B, et al. A one-year hospital-based prospective COVID-19 open-cohort in the Eastern Mediterranean region: The Khorshid COVID Cohort (KCC) study. medRxiv 2020; : 2020.05.11.20096727. 106 Almazeedi S, Youha SA, Jamal MH, et al. Clinical Characteristics, Risk Factors and Outcomes Among the First Consecutive 1,096 Patients Diagnosed with COVID-19: The Kuwait Experience. medRxiv 2020; : 2020.05.09.20096495. 107 Carrillo-Vega MF, Salinas-Escudero G, Garcia-Peña C, Gutierrez-Robledo LM, Parra-Rodriguez L. Early estimation of the risk factors for hospitalisation and mortality by COVID-19 in Mexico. medRxiv 2020; :2020.05.11.20098145.

108 Yanover C, Mizrahi B, Kalkstein N, et al. What factors increase the risk of complications in SARS-CoV-2 positive patients? A cohort study in a nationwide Israeli health organization. medRxiv 2020; : 2020.05.07.20091652.

109 Hamer M, Kivimäki M, Gale CR, Batty GD. Lifestyle risk factors, inflammatory mechanisms, and COVID-19 hospitalization: A community-based cohort study of 387,109 adults in UK. Brain Behav Immun 2020; 87: 184-7.

110 Regina J, Papadimitriou-Olivgeris M, Burger R, et al. Epidemiology, risk factors and clinical course of SARS-CoV-2 infected patients in a Swiss university hospital: an observational retrospective study. medRxiv 2020; : 2020.05.11.20097741.

111 de Lusignan S, Dorward J, Correa A, et al. Risk factors for SARS-CoV-2 among patients in the Oxford Royal College of General Practitioners Research and Surveillance Centre primary care network: a cross-sectional study. Lancet Infect Dis 2020; : S1473309920303716.

112 Targher G, Mantovani A, Wang X-B, et al. Patients with diabetes are at higher risk for severe illness from COVID19. Diabetes Metab 2020; published online May 13. DOI:10.1016/j.diabet.2020.05.001.

113 Valenti L, Bergna A, Pelusi S, et al. SARS-CoV-2 seroprevalence trends in healthy blood donors during the COVID-19 Milan outbreak. medRxiv 2020; : 2020.05.11.20098442.

114 Feuth T, Saaresranta T, Karlsson A, et al. Is sleep apnoea a risk factor for Covid-19? Findings from a retrospective cohort study. medRxiv 2020; : 2020.05.14.20098319.

115 Ge H, Zhu M, Du J, et al. Cardiac Structural and Functional Characteristics in Patients with Coronavirus Disease 2019: A Serial Echocardiographic Study. medRxiv 2020; :2020.05.12.20095885.

116 Parrotta E, Kister I, Charvet L, et al. COVID-19 outcomes in MS: Observational study of early experience from NYU Multiple Sclerosis Comprehensive Care Center. Neurol - Neuroimmunol Neuroinflammation 2020; 7: e835.

117 Shekhar R, Sheikh AB, Upadhyay S, Atencio J, Kapuria D. Early experience with COVID-19 patients at academic hospital in Southwestern United States. Infect Dis 2020; 52: 596-9.

118 Mejia-Vilet JM, Cordova-Sanchez BM, Fernandez-Camargo D, Mendez-Perez RA, Morales-Buenrostro LE, Hernandez-Gilsoul T. A Risk Score to Predict Admission to Intensive Care Unit in Patients With COVID-19: The ABCGOALS Score. medRxiv 2020; : 2020.05.12.20099416.

119 Chen C, Jiang J, Xu X, Hu Y, Hu Y, Zhao Y. Dynamic liver function indexes monitoring and clinical characteristics in three types of COVID-19 patients. medRxiv 2020; : 2020.05.13.20099614.

120 Li J, Chen Y, Chen S, et al. Derivation and validation of a prognostic model for predicting in-hospital mortality in patients admitted with COVID-19 in Wuhan, China: the PLANS (Platelet Lymphocyte Age Neutrophil Sex) model. medRxiv 2020; : 2020.05.13.20100370.

121 Palaiodimos L, Kokkinidis DG, Li W, et al. Severe obesity, increasing age and male sex are independently 
associated with worse in-hospital outcomes, and higher in-hospital mortality, in a cohort of patients with COVID-19 in the Bronx, New York. Metabolism 2020; 108: 154262.

122 Ip A, Berry DA, Hansen E, et al. Hydroxychloroquine and Tocilizumab Therapy in COVID-19 Patients - An Observational Study. medRxiv 2020; : 2020.05.21.20109207.

123 Heili-Frades S. COVID-19 Outcomes in 4712 consecutively confirmed SARS-CoV2 cases in the city of Madrid. medRxiv https://doi.org/10.1101/2020.05.22.20109850 (accessed July 27, 2020).

124 Vaquero LM, Barrado MES, Escobar D, et al. C-Reactive protein and SOFA score as early predictors of critical care requirement in patients with COVID-19 pneumonia in Spain. medRxiv 2020; : 2020.05.22.20110429.

125 Kim L, Garg S, O'Halloran A, et al. Interim Analysis of Risk Factors for Severe Outcomes among a Cohort of Hospitalized Adults Identified through the U.S. Coronavirus Disease 2019 (COVID-19)-Associated Hospitalization Surveillance Network (COVID-NET). medRxiv 2020; : 2020.05.18.20103390.

126 Wu MA, Fossali T, Pandolfi L, et al. COVID-19: the key role of pulmonary capillary leakage. An observational cohort study. medRxiv 2020; : 2020.05.17.20104877.

127 Shi Q, Zhao K, Yu J, et al. Clinical characteristics of 101 COVID-19 nonsurvivors in Wuhan, China: a retrospective study. medRxiv 2020; : 2020.03.04.20031039.

128 Al-Hindawi A, Sokhi J, Cuddihy J, et al. COVID-19 in London, a Case Series Demonstrating Late Improvement in Survivors. medRxiv 2020; : 2020.05.16.20103853.

129 Basse C, Diakite S, Servois V, et al. Characteristics and outcome of SARS-CoV-2 infection in cancer patients. medRxiv 2020; : 2020.05.14.20101576.

130 Freites D, Leon L, Mucientes A, et al. Risk factors for hospital admission related to COVID-19 in inflammatory rheumatic diseases. medRxiv 2020; : 2020.05.14.20101584.

131 Alshami AA, Alattas RA, Anan HF, et al. Silent Disease and Loss of Taste and Smell are Common Manifestations of SARS-COV-2 Infection in a Quarantine Facility: First report from Saudi Arabia. medRxiv 2020; : 2020.05.13.20100222. 132 Berumen J, Schmulson M, Alegre J, et al. Risk of infection and hospitalization by Covid-19 in Mexico: a casecontrol study. medRxiv 2020; : 2020.05.24.20104414.

133 Gianfrancesco M, Hyrich KL, Al-Adely S, et al. Characteristics associated with hospitalisation for COVID-19 in people with rheumatic disease: data from the COVID-19 Global Rheumatology Alliance physician-reported registry. Ann Rheum Dis 2020; 79: 859-66.

134 Li J, Long X, Zhu C, et al. Olfactory Dysfunction in Recovered Coronavirus Disease 2019 (COVID-19) Patients. Mov Disord; n/a. DOI:10.1002/mds.28172.

135 Batty GD, Deary I, Luciano M, Altschul D, Kivimaki M, Gale C. Psychosocial factors and hospitalisations for COVID19: Prospective cohort study of the general population. medRxiv 2020; : 2020.05.29.20100735.

136 Israel A, Feldhamer I, Lahad A, Levin-Zamir D, Lavie G. Smoking and the risk of COVID-19 in a large observational population study. medRxiv 2020; : 2020.06.01.20118877.

137 Valle DMD, Kim-schulze S, Hsin-hui H, et al. An inflammatory cytokine signature helps predict COVID-19 severity and death. medRxiv 2020; : 2020.05.28.20115758.

138 Chaudhry F, Bulka H, Rathnam AS, et al. COVID-19 in Multiple Sclerosis Patients and Risk Factors for Severe 
Infection. medRxiv 2020; : 2020.05.27.20114827.

139 Louis S, Dhawan A, Newey C, et al. Continuous Electroencephalography (cEEG) Characteristics and Acute Symptomatic Seizures in COVID-19 Patients. medRxiv 2020; : 2020.05.26.20114033.

140 Soto-Mota A, Garza BAM, Rodriguez EM, et al. THE LOW-HARM SCORE FOR PREDICTING MORTALITY IN PATIENTS DIAGNOSED WITH COVID-19: A MULTICENTRIC VALIDATION STUDY. medRxiv 2020; : 2020.05.26.20111120.

141 Garibaldi BT, Fiksel J, Muschelli J, et al. Patient trajectories and risk factors for severe outcomes among persons hospitalized for COVID-19 in the Maryland/DC region. medRxiv 2020; : 2020.05.24.20111864.

142 Docherty AB, Harrison EM, Green CA, et al. Features of 20133 UK patients in hospital with covid-19 using the ISARIC WHO Clinical Characterisation Protocol: prospective observational cohort study. BMJ 2020; 369. DOI:10.1136/bmj.m1985.

143 Boulware DR, Pullen MF, Bangdiwala AS, et al. A Randomized Trial of Hydroxychloroquine as Postexposure Prophylaxis for Covid-19. N Engl J Med 2020; published online June 3. DOI:10.1056/NEJMoa2016638.

144 Kuderer NM, Choueiri TK, Shah DP, et al. Clinical impact of COVID-19 on patients with cancer (CCC19): a cohort study. The Lancet 2020; 395: 1907-18.

145 Romão VC, Oliveira-Ramos F, Cruz-Machado AR, et al. A COVID-19 outbreak in a rheumatology department upon the early days of the pandemic. medRxiv 2020; : 2020.06.05.20107011.

146 Giannouchos T, Sussman R, Mier JM, Poulas K, Farsalinos K. Characteristics and risk factors for COVID-19 diagnosis and adverse outcomes in Mexico: an analysis of 89,756 laboratory-confirmed COVID-19 cases. medRxiv 2020; : 2020.06.04.20122481.

147 Ramlall V, Thangaraj P, Meydan C, et al. Identification of Immune complement function as a determinant of adverse SARS-CoV-2 infection outcome. medRxiv 2020; : 2020.05.05.20092452.

148 Wang B, Oekelen OV, Mouhieddine T, et al. A tertiary center experience of multiple myeloma patients with COVID19: lessons learned and the path forward. medRxiv 2020; : 2020.06.04.20122846.

149 Perrone F, Piccirillo MC, Ascierto PA, et al. Tocilizumab for patients with COVID-19 pneumonia. The TOCIVID-19 prospective phase 2 trial. medRxiv 2020; : 2020.06.01.20119149.

150 Sharma AK, Ahmed A, Baig VN, et al. Characteristics and Outcomes of Hospitalized Young Adults with Mild to Moderate Covid-19 at a University Hospital in India. medRxiv 2020; : 2020.06.02.20106310.

151 Eugen-Olsen J, Altintas I, Tingleff J, et al. Low levels of the prognostic biomarker suPAR are predictive of mild outcome in patients with symptoms of COVID-19 - a prospective cohort study. medRxiv 2020; : 2020.05.27.20114678. 152 Martinez-Portilla RJ, Sotiriadis A, Torres-Torres J, et al. Risk factors for mortality in pregnant women with SARSCoV-2 infection. medRxiv 2020; : 2020.05.31.20107276.

153 Raisi-Estabragh Z, McCracken C, Bethell MS, et al. Greater risk of severe COVID-19 in Black, Asian and Minority Ethnic populations is not explained by cardiometabolic, socioeconomic or behavioural factors, or by $25(\mathrm{OH})$-vitamin $\mathrm{D}$ status: study of 1326 cases from the UK Biobank. J Public Health DOI:10.1093/pubmed/fdaa095.

154 Luo H, Liu S, Wang Y, et al. Age differences in clinical features and outcomes in patients with COVID-19, Jiangsu, China: a retrospective, multi-center cohort study. medRxiv 2020; : 2020.06.01.20086025. 
155 Houlihan CF, Vora N, Byrne T, et al. Pandemic peak SARS-CoV-2 infection and seroconversion rates in London frontline health-care workers. The Lancet 2020; : S0140673620314847.

156 Cen $Y$, Chen X, Shen Y, et al. Risk factors for disease progression in patients with mild to moderate coronavirus disease 2019_a multi-centre observational study. Clin Microbiol Infect 2020; : S1198743X20303414.

157 Klang E, Kassim G, Soffer S, Freeman R, Levin MA, Reich DL. Morbid Obesity as an Independent Risk Factor for COVID-19 Mortality in Hospitalized Patients Younger than 50. Obesity; n/a. DOI:10.1002/oby.22913.

158 Maraschini A, Corsi E, Salvatore MA, Donati S. Coronavirus and birth in Italy: results of a national population-based cohort study. medRxiv 2020; : 2020.06.11.20128652.

159 Wang A-L, Zhong X, Hurd Y. Comorbidity and Sociodemographic determinants in COVID-19 Mortality in an US Urban Healthcare System. medRxiv 2020; : 2020.06.11.20128926.

160 McQueenie R, Foster H, Jani BD, et al. Multimorbidity, Polypharmacy, and COVID-19 infection within the UK Biobank cohort. medRxiv 2020; : 2020.06.10.20127563.

161 Apea VJ, Wan YI, Dhairyawan R, et al. Ethnicity and outcomes in patients hospitalised with COVID-19 infection in East London: an observational cohort study. medRxiv 2020; : 2020.06.10.20127621.

162 Woolford SJ, D'angelo S, Curtis EM, et al. COVID-19 and associations with frailty and multimorbidity: a prospective analysis of UK Biobank participants. medRxiv 2020; : 2020.06.09.20126292.

163 Hultcrantz M, Richter J, Rosenbaum C, et al. COVID-19 infections and outcomes in patients with multiple myeloma in New York City: a cohort study from five academic centers. medRxiv 2020; : 2020.06.09.20126516.

164 Rajter JC, Sherman M, Fatteh N, Vogel F, Sacks J, Rajter J-J. ICON (Ivermectin in COvid Nineteen) study: Use of Ivermectin is Associated with Lower Mortality in Hospitalized Patients with COVID19. medRxiv 2020; : 2020.06.06.20124461.

165 Lan F-Y, Suharlim C, Kales SN, Yang J. Association between SARS-CoV-2 infection, exposure risk and mental health among a cohort of essential retail workers in the United States. medRxiv 2020; : 2020.06.08.20125120.

166 Zeng H, Zhang T, He X, et al. Impact of Chronic Comorbidities on Progression and Prognosis in Patients with COVID-19: A Retrospective Cohort Study in 1031 Hospitalized Cases in Wuhan, China. medRxiv 2020; : 2020.06.14.20125997.

167 Suleyman G, Fadel RA, Malette KM, et al. Clinical Characteristics and Morbidity Associated With Coronavirus Disease 2019 in a Series of Patients in Metropolitan Detroit. JAMA Netw Open 2020; 3: e2012270-e2012270.

168 Chen L, Yu J, He W, et al. Risk factors for death in 1859 subjects with COVID-19. Leukemia 2020; : 1-11.

169 Garassino MC, Whisenant JG, Huang L-C, et al. COVID-19 in patients with thoracic malignancies (TERAVOLT): first results of an international, registry-based, cohort study. Lancet Oncol 2020; 21: 914-22.

170 Hernández-Garduño E. Obesity is the comorbidity more strongly associated for Covid-19 in Mexico. A case-control study. Obes Res Clin Pract2020; published online June 12. DOI:10.1016/j.orcp.2020.06.001.

171 Govind R, Freitas DF de, Pritchard MR, Hayes RD, MacCabe JH. Clozapine treatment and risk of COVID-19. medRxiv 2020; : 2020.06.17.20133595.

172 Sisó-Almirall A, Kostov B, Mas-Heredia M, et al. PROGNOSTIC FACTORS IN SPANISH COVID-19 PATIENTS: A CASE SERIES FROM BARCELONA. medRxiv 2020; : 2020.06.18.20134510. 
173 Gu T, Mack JA, Salvatore M, et al. COVID-19 outcomes, risk factors and associations by race: a comprehensive analysis using electronic health records data in Michigan Medicine. medRxiv 2020; : 2020.06.16.20133140.

174 Kibler M, Carmona A, Marchandot B, et al. Risk and severity of COVID-19 and ABO blood group in transcatheter aortic valve patients. medRxiv 2020; : 2020.06.13.20130211.

175 Ikitimur H, Uysal BB, Cengiz M, et al. "Determining Host Factors Contributing to Disease Severity in a Family Cluster of 29 Hospitalized SARS-CoV-2 Patients: Could Genetic Factors Be Relevant in the Clinical Course of COVID19?". J Med Virol; n/a. DOI:10.1002/jmv.26106.

176 Sierpiński R, Pinkas J, Jankowski M, et al. Sex differences in the frequency of gastrointestinal symptoms and olfactory or taste disorders among 1,942 non-hospitalized patients with COVID-19. Pol Arch Intern Med 2020; published online June 3. DOI:10.20452/pamw.15414.

177 Zhou Y, He X, Zhang J, et al. Prolonged SARS-CoV-2 Viral Shedding in Patients with COVID-19 was Associated with Delayed Initiation of Arbidol Treatment: a retrospective cohort study. medRxiv 2020; : 2020.06.09.20076646.

178 Crovetto F, Crispi F, Llurba E, Figueras F, Gomez-Roig MD, Gratacos E. SEROPREVALENCE AND CLINICAL SPECTRUM OF SARS-CoV-2 INFECTION IN THE FIRST VERSUS THIRD TRIMESTER OF PREGNANCY. medRXIV 2020; : 2020.06.17.20134098.

179 Veras FP, Pontelli M, Silva C, et al. SARS-CoV-2 triggered neutrophil extracellular traps (NETs) mediate COVID19 pathology. medRxiv 2020; : 2020.06.08.20125823.

180 Sterlin D, Mathian A, Miyara M, et al. IgA dominates the early neutralizing antibody response to SARS-CoV-2. medRxiv 2020; : 2020.06.10.20126532.

181 Rossi B, Nguyen LS, Zimmermann P, et al. Effect of tocilizumab in hospitalized patients with severe pneumonia COVID-19: a cohort study. medRxiv 2020; : 2020.06.06.20122341.

182 Duan L, Zhang S, Guo M, et al. Epidemiological and clinical characteristics in patients with SARS-CoV-2 antibody negative probable COVID-19 in Wuhan. medRxiv 2020; : 2020.06.18.20134619.

183 Martin-Jimenez P, Munoz-Garcia MI, Seoane D, et al. Cognitive impairment is a common comorbidity in COVID-19 deceased patients. A hospital-based retrospective cohort study. medRxiv 2020; : 2020.06.08.20125872.

184 Elezkurtaj S, Greuel S, Ihlow J, et al. Causes of Death and Comorbidities in Patients with COVID-19. medRxiv 2020; : 2020.06.15.20131540.

185 Lenka J, Chhabria MS, Sharma N, et al. Clinical characteristics and outcomes of critically ill patients with COVID19 in a tertiary community hospital in upstate New York. medRxiv 2020; : 2020.06.18.20135046.

186 Olivares F, Munoz D, Fica A, et al. Covid-19 in Chile. The experience of a Regional reference Center. Preliminary report. medRxiv 2020; : 2020.06.14.20130898.

187 Salton F, Confalonieri P, Santus $\mathrm{P}$, et al. Prolonged low-dose methylprednisolone in patients with severe COVID19 pneumonia. medRxiv 2020; : 2020.06.17.20134031.

188 Wei W, Ortwine JK, Mang NS, Joseph C, Hall BC, Prokesch BC. Limited Role for Antibiotics in COVID-19: Scarce Evidence of Bacterial Coinfection. medRxiv 2020; : 2020.06.16.20133181.

189 Zuo Y, Estes SK, Gandhi AA, et al. Prothrombotic antiphospholipid antibodies in COVID-19. medRxiv 2020; : 2020.06.15.20131607. 
190 Killerby ME. Characteristics Associated with Hospitalization Among Patients with COVID-19 - Metropolitan Atlanta, Georgia, March-April 2020. MMWR Morb Mortal Wkly Rep 2020; 69. DOI:10.15585/mmwr.mm6925e1.

191 Petrilli CM, Jones SA, Yang J, et al. Factors associated with hospital admission and critical illness among 5279 people with coronavirus disease 2019 in New York City: prospective cohort study. BMJ 2020; 369.

DOI:10.1136/bmj.m1966.

192 Magagnoli J, Narendran S, Pereira F, et al. Outcomes of Hydroxychloroquine Usage in United States Veterans Hospitalized with COVID-19. Med 2020; : S2666634020300064.

193 Bello-Chavolla OY, Bahena-López JP, Antonio-Villa NE, et al. Predicting Mortality Due to SARS-CoV-2: A Mechanistic Score Relating Obesity and Diabetes to COVID-19 Outcomes in Mexico. J Clin Endocrinol Metab $2020 ; 105$. DOI:10.1210/clinem/dgaa346.

194 Zuo Y, Zuo M, Yalavarthi S, et al. Neutrophil extracellular traps and thrombosis in COVID-19. medRxiv 2020; : 2020.04.30.20086736.

195 Sigel K, Swartz T, Golden E, et al. Covid-19 and People with HIV Infection: Outcomes for Hospitalized Patients in New York City. Clin Infect Dis DOI:10.1093/cid/ciaa880.

196 Nguyen AB, Upadhyay GA, Chung B, et al. Outcomes and Cardiovascular Comorbidities in a Predominantly African-American Population with COVID-19. medRxiv 2020; : 2020.06.28.20141929.

197 Melo AC de, Thuler LCS, Silva JL da, et al. Cancer inpatient with COVID-19: a report from the Brazilian National Cancer Institute. medRxiv 2020; : 2020.06.27.20141499.

198 Auvinen R, Nohynek H, Syrjänen R, et al. Comparison of the clinical characteristics and outcomes of hospitalized adult COVID-19 and influenza patients: a prospective observational study. medRxiv 2020; : 2020.06.29.20140632.

199 Souza FSH, Hojo-Souza NS, Santos EB, Silva CM, Guidoni DL. Predicting the disease outcome in COVID-19 positive patients through Machine Learning: a retrospective cohort study with Brazilian data. medRxiv 2020; : 2020.06.26.20140764.

200 Mendy A, Apewokin S, Wells AA, Morrow AL. Factors Associated with Hospitalization and Disease Severity in a Racially and Ethnically Diverse Population of COVID-19 Patients. medRxiv 2020; : 2020.06.25.20137323.

201 Pongpirul WA, Wiboonchutikul S, Charoenpong L, et al. Clinical course and potential predicting factors of pneumonia of adult patients with coronavirus disease 2019 (COVID-19): A retrospective observational analysis of 193 confirmed cases in Thailand. medRxiv 2020; : 2020.06.24.20139642.

202 Jin C, Gu J, Yuan Y, et al. Treatment of Six COVID-19 Patients with Convalescent Plasma. medRxiv 2020; : 2020.05.21.20109512.

203 Favara DM, Cooke A, Doffinger R, et al. First results from the UK COVID-19 Serology in Oncology Staff Study (CSOS). medRxiv 2020; : 2020.06.22.20136838.

204 Fisman D, Greer AL, Tuite A. Derivation and Validation of Clinical Prediction Rule for COVID-19 Mortality in Ontario, Canada. medRxiv 2020; : 2020.06.21.20136929.

205 Madariaga MLL, Guthmiller J, Schrantz S, et al. Clinical predictors of donor antibody titer and correlation with recipient antibody response in a COVID-19 convalescent plasma clinical trial. medRxiv 2020; : 2020.06.21.20132944. 206 Senkal N. Chronic ACE Inhibitor use is Associated with Decreased Odds of Severe Disease in Patients with 
COVID-19. Anatol J Cardiol 2020. DOI:10.14744/AnatolJCardiol.2020.57431.

207 Mohamud AY, Griffith B, Rehman M, et al. Intraluminal Carotid Artery Thrombus in COVID-19: Another Danger of Cytokine Storm? Am J Neuroradiol 2020; published online July 2. DOI:10.3174/ajnr.A6674.

208 Magleby R, Westblade LF, Trzebucki A, et al. Impact of SARS-CoV-2 Viral Load on Risk of Intubation and Mortality Among Hospitalized Patients with Coronavirus Disease 2019. Clin Infect Dis DOI:10.1093/cid/ciaa851.

209 Kimmig LM, Wu D, Gold M, et al. IL6 inhibition in critically ill COVID-19 patients is associated with increased secondary infections. medRxiv 2020; : 2020.05.15.20103531.

210 Bello-Chavolla OY, Antonio-Villa NE, Vargas-Vázquez A, Fermín-Martínez CA, Márquez-Salinas A, Bahena-López JP. Profiling pre-symptomatic and asymptomatic cases with confirmed SARS-CoV-2 infection in Mexico City. medRxiv 2020; : 2020.07.02.20145516.

211 Zacharioudakis IM, Prasad PJ, Zervou FN, et al. Association of SARS-CoV-2 Genomic Load with COVID-19 Patient Outcomes. medRxiv 2020; : 2020.07.02.20145151.

212 Antonio-Villa NE, Bello-Chavolla OY, Vargas-Vazquez A, Fermin-Martinez CA, Marquez-Salinas A, Bahena-Lopez JP. Health-care workers with COVID-19 living in Mexico City: clinical characterization and related outcomes. medRxiv 2020; : 2020.07.02.20145169.

213 Patel M, Chowdhury J, Mills N, et al. ROX Index Predicts Intubation in Patients with COVID-19 Pneumonia and Moderate to Severe Hypoxemic Respiratory Failure Receiving High Flow Nasal Therapy. medRxiv 2020; :

2020.06.30.20143867.

214 Merzon E, Tworowski D, Gorohovski A, et al. Low plasma 25(OH) vitamin D3 level is associated with increased risk of COVID-19 infection: an Israeli population-based study. medRxiv 2020; : 2020.07.01.20144329.

215 Fan X, Yin C, Wang J, et al. Pre-diagnostic circulating concentrations of insulin-like growth factor-1 and risk of COVID-19 mortality: results from UK Biobank. medRxiv 2020; : 2020.07.09.20149369.

216 Shi Z, Resurreccion WK, Wang C-H, et al. Association of Cancer with Risk and Mortality of COVID-19: Results from the UK Biobank. medRxiv 2020; : 2020.07.10.20151076.

217 Maucourant C, Filipovic I, Ponzetta A, et al. Natural killer cell activation related to clinical outcome of COVID-19. medRxiv 2020; : 2020.07.07.20148478.

218 Elmunzer BJ, Spitzer RL, Foster LD, et al. Digestive Manifestations in Patients Hospitalized with COVID-19. medRxiv 2020; : 2020.07.07.20143024.

219 Alizadehsani R, Sani ZA, Behjati M, et al. Risk Factors Prediction, Clinical Outcomes, and Mortality of COVID-19 Patients. medRxiv 2020; : 2020.07.07.20148569.

220 Xie Y, Chen S, Wang X, et al. Early Diagnosis and Clinical Significance of Acute Cardiac Injury - Under the Iceberg: A Retrospective Cohort Study of 619 Non-critically III Hospitalized COVID-19 Pneumonia Patients. medRxiv $2020 ;:$ : 2020.07.06.20147256.

221 Fox TA, Troy-Barnes E, Kirkwood AA, et al. Clinical outcomes and risk factors for severe COVID-19 infection in patients with haematological disorders receiving chemo- or immunotherapy. Br J Haemato/; n/a. DOI:10.1111/bjh.17027. 222 Martinez-Resendez MF, Castilleja-Leal F, Torres-Quintanilla A, et al. Initial experience in Mexico with convalescent plasma in COVID-19 patients with severe respiratory failure, a retrospective case series. medRxiv 2020; : 
2020.07.14.20144469.

223 Hoertel N, Rico MS, Vernet R, et al. Observational Study of Haloperidol in Hospitalized Patients with Covid-19. medRxiv 2020; : 2020.07.15.20150490.

224 McGrail DE, Edwards D. COVID-19 Case Series at UnityPoint Health St. Lukes Hospital in Cedar Rapids, IA. medRxiv 2020; : 2020.07.17.20156521.

225 Pandolfi L, Fossali T, Frangipane V, et al. Broncho-alveolar inflammation in COVID-19 patients: a correlation with clinical outcome. medRxiv 2020; : 2020.07.17.20155978.

226 Kazuyoshi Kurashima, Naho Kagiyama, Takashi Ishiguro, et al. IgG antibody seroconversion and the clinical progression of COVID-19 pneumonia: A retrospective, cohort study | medRxiv. https://doi.org/10.1101/2020.07.16.20154088 (accessed Aug 25, 2020).

227 Zhan Z, Yang X, Du H, et al. Early Improvement of Acute Respiratory Distress Syndrome in Patients with COVID19: Insights from the Data of ICU Patients in Chongqing, China. medRxiv 2020; : 2020.07.15.20154047.

228 Omrani AS, Almaslamani MA, Daghfal J, et al. The First Consecutive 5000 Patients with Coronavirus Disease 2019 from Qatar; a Nation-wide Cohort Study. medRxiv 2020; : 2020.07.15.20154690.

229 Gupta R, Agrawal R, Bukhari Z, et al. Higher Comorbidities and Early Death is Characteristic of Hospitalized African-American Patients with COVID-19. medRxiv 2020; : 2020.07.15.20154906.

230 Hussein MH, Toraih EA, Attia AS, et al. Asthma in COVID-19: An extra chain fitting around the neck? medRxiv 2020; : 2020.07.13.20153130.

231 Bian H, Zheng Z-H, Wei D, et al. Meplazumab treats COVID-19 pneumonia: an open-labelled, concurrent controlled add-on clinical trial. medRxiv 2020; : 2020.03.21.20040691.

232 Eiros R, Barreiro-Perez M, Martin-Garcia A, et al. Pericarditis and myocarditis long after SARS-CoV-2 infection: a cross-sectional descriptive study in health-care workers. medRxiv 2020; : 2020.07.12.20151316.

233 Marcos M, Belhassen-Garcia M, Puente AS-, et al. Development of a severity of disease score and classification model by machine learning for hospitalized COVID-19 patients. medRxiv 2020; : 2020.07.13.20150177.

234 Hoertel N, Rico MS, Vernet R, et al. Association between SSRI Antidepressant Use and Reduced Risk of Intubation or Death in Hospitalized Patients with Coronavirus Disease 2019: a Multicenter Retrospective Observational Study. medRxiv 2020; : 2020.07.09.20143339.

235 Soares R de CM, Mattos LR, Raposo LM. Risk Factors for Hospitalization and Mortality due to COVID-19 in Espírito Santo State, Brazil. 2020; : tpmd200483.

236 Zobairy H, Shamsoddin E, Rasouli MA, et al. Association of olfactory dysfunction with hospitalization for COVID-19: a multicenter study in Kurdistan. medRxiv 2020; : 2020.07.26.20158550.

237 Altamimi H, Alahmad Y, Khazal F, et al. The Outcome of COVID-19 Patients with Acute Myocardial Infarction. medRxiv 2020; : 2020.07.21.20156349.

238 Thompson JV, Meghani N, Powell BM, et al. Patient characteristics and predictors of mortality in 470 adults admitted to a district general hospital in England with Covid-19. medRxiv 2020; : 2020.07.21.20153650.

239 Reiter T, Pajenda S, Wagner L, et al. Covid-19 serology in nephrology health care workers. medRxiv 2020; : 2020.07.21.20136218. 
240 Motta JK, Ogunnaike RO, Shah R, et al. Clinical Outcomes With the Use of Prophylactic Versus Therapeutic Anticoagulation in COVID-19. medRxiv 2020; :2020.07.20.20147769.

241 Santos C, Rhee Y, Hollinger E, et al. Comparative Incidence and Outcomes of COVID-19 in Kidney or KidneyPancreas Transplant Recipients Versus Kidney or Kidney-Pancreas Waitlisted Patients: A Pilot Study. medRxiv 2020; : 2020.07.20.20157990.

242 Schneeweiss MC, Leonard S, Weckstein A, Schneeweiss S, Rassen J. Renin-Angiotensin-Aldosterone-System inhibitor use in patients with COVID-19 infection and prevention of serious events: a cohort study in commercially insured patients in the US. medRxiv 2020; : 2020.07.22.20159855.

\section{Concha-Mejia A, Rincon-Sanchez RA. CCOFEE-GI Study: Colombian COVID19 First Experience in}

Gastroentrology. Characterization of digestive manifestations in patients diagnosed with COVID-19 at a highly complex institution in Bogota D.C., Colombia. medRxiv 2020; : 2020.07.24.20161604.

244 Izquierdo JL, Almonacid C, Gonzalez Y, et al. The impact of COVID-19 on patients with asthma. medRxiv 2020; : 2020.07.24.20161596.

245 Bernaola N, Mena R, Bernaola A, et al. Observational Study of the Efficiency of Treatments in Patients Hospitalized with Covid-19 in Madrid. medRxiv 2020; : 2020.07.17.20155960.

246 Qi D, Yan X, Tang X, et al. Epidemiological and clinical features of 2019-nCoV acute respiratory disease cases in Chongqing municipality, China: a retrospective, descriptive, multiple-center study. medRxiv 2020; :2020.03.01.20029397. 247 Peters EJ, Collard D, Assen S van, et al. Outcomes of Persons With COVID-19 in Hospitals With and Without Standard Treatment With (Hydroxy)chloroquine. medRxiv 2020; : 2020.08.14.20173369.

248 Ouyang J, Shan X, Wang X, et al. Clinical characteristics of COVID-19 and the model for predicting the occurrence of critically ill patients: a retrospective cohort study. medRxiv 2020; : 2020.08.13.20173799.

249 Valenzuela O, Ibanez SE, Poli M, et al. First report of tocilizumab use in a cohort of Latin American patients hospitalized for severe COVID-19 pneumonia. medRxiv 2020; : 2020.08.12.20173104.

250 Monteiro ACC, Suri R, Emeruwa IO, et al. Obesity and Smoking as Risk Factors for Invasive Mechanical Ventilation in COVID-19: a Retrospective, Observational Cohort Study. medRxiv 2020; : 2020.08.12.20173849.

251 Philipose Z, Smati N, Wong CSJ, Aspey K, Mendall MA. Obesity, old age and frailty are the true risk factors for COVID-19 mortality and not chronic disease or ethnicity in Croydon. medRxiv 2020; : 2020.08.12.20156257.

252 Weerahandi $\mathrm{H}$, Hochman KA, Simon E, et al. Post-discharge health status and symptoms in patients with severe COVID-19. medRxiv 2020; : 2020.08.11.20172742.

253 Altibi AM, Bhargava P, Liaqat H, et al. Comparative Clinical Outcomes and Mortality in Prisoner and Non-Prisoner Populations Hospitalized with COVID-19: A Cohort from Michigan. medRxiv 2020; : 2020.08.08.20170787.

254 Izzi-Engbeaya C, Distaso W, Amin A, et al. Severe COVID-19 and Diabetes: A Retrospective Cohort Study from Three London Teaching Hospitals. medRxiv 2020; : 2020.08.07.20160275.

255 Rizzo S, Chawla D, Zalocusky K, et al. Descriptive epidemiology of 16,780 hospitalized COVID-19 patients in the United States. medRxiv 2020; : 2020.07.17.20156265.

256 Dashti HT, Bates D, Fiskio JM, Roche EC, Mora S, Demler O. Clinical Characteristics and Severity of COVID-19 Disease in Patients from Boston Area Hospitals. medRxiv 2020; : 2020.07.27.20163071. 
257 Morshed MS, Mosabbir AA, Chowdhury P, Ashadullah SM, Hossain MS. Clinical manifestations of patients with Coronavirus Disease 2019 (COVID- 19) attending at hospitals in Bangladesh. medRxiv 2020; : 2020.07.30.20165100. 258 Jun T, Nirenberg S, Kovatch P, Huang K. Sex-specificity of mortality risk factors among hospitalized COVID-19 patients in New York City: prospective cohort study. medRxiv 2020; : 2020.07.29.20164640.

259 Higuchi T, Nishida T, Iwahashi H, et al. Early Clinical Factors Predicting the Development of Critical Disease in Japanese Patients with COVID-19: A Single-Center Retrospective, Observational Study. medRxiv 2020; : 2020.07.29.20159442.

260 Zhou K, Sun Y, Li L, et al. Eleven Routine Clinical Features Predict COVID-19 Severity. medRxiv 2020; : 2020.07.28.20163022.

261 Salerno S, Zhao Z, Sankar SP, et al. Understanding the patterns of repeated testing for COVID-19: Association with patient characteristics and outcomes. medRxiv 2020; : 2020.07.26.20162453.

262 Kumar A, Prasad G, Srivastav S, Gautam VK, Sharma N. A Retrospective Study on Efficacy and Safety of Guduchi Ghan Vati for Covid-19 Asymptomatic Patients. medRxiv 2020; : 2020.07.23.20160424.

263 Hao S-R, Zhang S-Y, Lian J-S, et al. Liver Enzyme Elevation in Coronavirus Disease 2019: A Multicenter, Retrospective, Cross-Sectional Study. Am J Gastroenterol 2020; published online June 1.

DOI:10.14309/ajg.0000000000000717.

264 Iversen K, Bundgaard H, Hasselbalch RB, et al. Risk of COVID-19 in health-care workers in Denmark: an observational cohort study. Lancet Infect Dis 2020; 0. DOI:10.1016/S1473-3099(20)30589-2.

265 Hippisley-Cox J, Young D, Coupland C, et al. Risk of severe COVID-19 disease with ACE inhibitors and angiotensin receptor blockers: cohort study including 8.3 million people. Heart2020; : heartjnl-2020-317393.

266 Fillmore NR, La J, Szalat RE, et al. Prevalence and outcome of Covid-19 infection in cancer patients: a national VA study. medRxiv 2020; : 2020.08.21.20177923.

267 Rashid M, Wu J, Timmis A, et al. Clinical Characteristics and Outcomes of COVID-19 Positive Acute Coronary Syndrome Patients; a multisource Electronic Healthcare Records Study from England. medRxiv 2020; : 2020.08.20.20175091.

268 Pan A, Khan O, Meeks J, et al. Disparities in COVID-19 Hospitalizations and Mortality among Black and Hispanic Patients: Cross-Sectional Analysis from the Greater Houston Metropolitan Area. medRxiv 2020; : 2020.08.19.20177956.

269 Alkurt G, Murt A, Aydin Z, et al. Seroprevalence of Coronavirus Disease 2019 (COVID-19) Among Health Care Workers from Three Pandemic Hospitals of Turkey. medRxiv 2020; : 2020.08.19.20178095.

270 Zhao Z, Chen A, Hou W, et al. Prediction model and risk scores of ICU admission and mortality in COVID-19. PLOS ONE 2020; 15: e0236618.

271 Holman N, Knighton P, Kar P, et al. Risk factors for COVID-19-related mortality in people with type 1 and type 2 diabetes in England: a population-based cohort study. Lancet Diabetes Endocrinol 2020; 0. DOI:10.1016/S22138587(20)30271-0.

272 Qu J, Chang LK, Tang X, et al. Clinical characteristics of COVID-19 and its comparison with influenza pneumonia. Acta Clin Belg 2020; 0: 1-9.

273 Chand S, Kapoor S, Orsi D, et al. COVID-19-Associated Critical IIIness-Report of the First 300 Patients Admitted 
to Intensive Care Units at a New York City Medical Center: J Intensive Care Med 2020; published online Aug 19. DOI:10.1177/0885066620946692.

274 Oliveira E, Parikh A, Lopez-Ruiz A, et al. ICU Outcomes and Survival in Patients with Severe COVID-19 in the Largest Health Care System in Central Florida. medRxiv 2020; : 2020.08.25.20181909.

275 Mohamed-Hussein A, Galal I, Saad M, et al. Post-COVID-19 Functional Status: Relation to age, smoking, hospitalization and comorbidities. medRxiv 2020; : 2020.08.26.20182618.

276 Villar-Garcia J, Vivanco-Hidalgo RM, Cleries M, et al. Risk factors for SARS-CoV-2 infection, hospitalisation, and death in Catalonia, Spain: a population-based cross-sectional study. medRxiv 2020; : 2020.08.26.20182303.

277 Ibarra-Nava I, Flores-Rodriguez KG, Ruiz-Herrera V, et al. Ethnic disparities in COVID-19 mortality in Mexico: a cross-sectional study based on national data. medRxiv 2020; : 2020.08.26.20182543.

278 Ibrahim D, Dulipsingh L, Zapatka L, et al. Factors Associated with Good Patient Outcomes Following Convalescent Plasma in COVID-19: A Prospective Phase II Clinical Trial. medRxiv 2020; : 2020.08.27.20183293.

279 Rubio-Rivas M, Ronda M, Padulles A, et al. Beneficial Effect of Corticosteroids in Preventing Mortality in Patients Receiving Tocilizumab to Treat Severe COVID-19 IIIness. medRxiv 2020; : 2020.08.31.20182428.

280 Mamtani M, Athavale AM, Abraham M, et al. ASSOCIATION OF HYPERGLYCEMIA WITH HOSPITAL MORTALITY IN COVID-19 PATIENTS WITHOUT DIABETES: A COHORT STUDY. medRxiv 2020; : 2020.08.31.20185157.

281 Ren HG, Guo X, Blighe K, et al. Risk Factors for ICU Admission, Mechanical Ventilation and Mortality in Hospitalized Patients with COVID-19 in Hubei, China. medRxiv 2020; : 2020.08.31.20184952.

282 Yoo E, Percha B, Tomlinson M, et al. Development and calibration of a simple mortality risk score for hospitalized COVID-19 adults. medRxiv 2020; : 2020.08.31.20185363.

283 Mutambudzi M, Niedzwiedz CL, Macdonald EB, et al. Occupation and risk of severe COVID-19: prospective cohort study of 120,075 UK Biobank participants. medRxiv 2020; : 2020.05.22.20109892.

284 Yan H, Valdes AM, Vijay A, et al. Role of Drugs used for chronic disease management on Susceptibility and Severity of COVID-19: A Large Case-Control Study. medRxiv 2020; : 2020.04.24.20077875.

285 Mancilla-Galindo J, Vera-Zertuche JM, Navarro-Cruz AR, et al. Development and Validation of the Patient History COVID-19 (PH-Covid19) Scoring System: A Multivariable Prediction Model of Death in Mexican Patients with COVID-19. medRxiv 2020; : 2020.09.05.20189142.

286 Ullah AZMD, Sivapalan L, Chelala C, Kocher HM. COVID-19 in patients with hepatobiliary and pancreatic diseases in East London: A single-centre cohort study. medRxiv 2020; : 2020.09.07.20189621.

287 Nicholson CJ, Wooster L, Sigurslid HH, et al. Estimating Risk of Mechanical Ventilation and Mortality Among Adult COVID-19 patients Admitted to Mass General Brigham: The VICE and DICE Scores. medRxiv 2020; : 2020.09.14.20194670.

288 Ariza BE, Torres YX, Salgado D, et al. Seroprevalence and seroconversion rates to SARS-CoV-2 in interns, residents, and medical doctors in a University Hospital in Bogota, Colombia. medRxiv 2020; : 2020.09.15.20195313. 289 Zhu S, Gao Q, Yang L, et al. Prevalence and risk factors of disability and anxiety in a retrospective cohort of 432 survivors of Coronavirus Disease-2019 (Covid-19) from China. medRxiv 2020; : 2020.08.26.20182246. 
290 Sun L, Sanjna S, Le A, et al. Rates of COVID-19-related Outcomes in Cancer compared to non-Cancer Patients. medRxiv 2020; : 2020.08.14.20174961.

291 Kalan ME, Ghobadi H, Taleb ZB, et al. Descriptive characteristics of hospitalized adult smokers and never-smokers with COVID-19. Tob Induc Dis 2020; 18. DOI:10.18332/tid/122759.

292 Burrell AJ, Pellegrini B, Salimi F, et al. Outcomes of COVID-19 patients admitted to Australian intensive care units during the early phase of the pandemic. Med J Aust 2020; : 1.

293 ISARIC (International Severe Acute Respiratory and Emerging Infections Consortium). ; : 34.

294 Meini S, Fortini A, Andreini R, Sechi LA, Tascini C. The Paradox of the Low Prevalence of Current Smokers Among Covid-19 Patients Hospitalized in Non-Intensive Care Wards: Results From an Italian Multicenter Case-Control Study.

Nicotine Tob Res DOl:10.1093/ntr/ntaa188.294 Meini S, Fortini A, Andreini R, Sechi LA, Tascini C. The Paradox of the Low Prevalence of Current Smokers Among Covid-19 Patients Hospitalized in Non-Intensive Care Wards: Results From an Italian Multicenter Case-Control Study. Nicotine Tob Res DOI:10.1093/ntr/ntaa188.269 Chand S, Kapoor S, Orsi D, et al. COVID-19-Associated Critical IIIness-Report of the First 300 Patients Admitted to Intensive Care Units at a New York City Medical Center: J Intensive Care Med 2020; published online Aug 19. DOI:10.1177/0885066620946692. 